\title{
CONTROLLABLITY OF A QUANTUM PARTICLE IN A 1D VARIABLE DOMAIN
}

\author{
KARINE BEAUCHARD ${ }^{1}$
}

\begin{abstract}
We consider a quantum particle in a $1 \mathrm{D}$ infinite square potential well with variable length. It is a nonlinear control system in which the state is the wave function $\phi$ of the particle and the control is the length $l(t)$ of the potential well. We prove the following controllability result : given $\phi_{0}$ close enough to an eigenstate corresponding to the length $l=1$ and $\phi_{f}$ close enough to another eigenstate corresponding to the length $l=1$, there exists a continuous function $l:[0, T] \rightarrow \mathbb{R}_{+}^{*}$ with $T>0$, such that $l(0)=1$ and $l(T)=1$, and which moves the wave function from $\phi_{0}$ to $\phi_{f}$ in time $T$. In particular, we can move the wave function from one eigenstate to another one by acting on the length of the potential well in a suitable way. Our proof relies on local controllability results proved with moment theory, a Nash-Moser implicit function theorem and expansions to the second order.
\end{abstract}

Mathematics Subject Classification. 35B37, 35Q55, 93B05, 93C20.

Received January 2, 2006. Revised July 10, 2006.

Published online September 21, 2007.

\section{INTRODUCTION}

\subsection{Main result}

We consider a quantum particle in a potential well with variable length $l(\tau)$, where

$$
\begin{aligned}
l:[0,+\infty) & \rightarrow(0,+\infty) \\
\tau & \mapsto l(\tau)
\end{aligned}
$$

is a continuous function of the time variable $\tau$. At any time $\tau$, the particle is represented by a wave function $\phi(\tau, z)$,

$$
\begin{aligned}
& \phi:[0,+\infty) \times(0, l(\tau)) \rightarrow \mathbb{C} \\
& (\tau \quad, \quad z) \quad \mapsto \phi(\tau, z)
\end{aligned}
$$

where $z$ is the space variable. The physical meaning of $|\phi(\tau, z)|^{2} \mathrm{~d} z$ is the probability of the particle to be in an elementary volume $\mathrm{d} z$ surrounding the position $z$ at time $\tau$, thus, at any time $\tau$, the wave function defines

Keywords and phrases. Controllability, Schrödinger equation, Nash-Moser theorem, moment theory.

1 CMLA, ENS Cachan, 61 avenue du président Wilson, 94235 Cachan cedex, France; Karine.Beauchard@cmla.ens-cachan.fr 
a point on the $L^{2}((0, l(\tau)), \mathbb{C})$-sphere

$$
\int_{0}^{l(\tau)}|\phi(\tau, z)|^{2} \mathrm{~d} z=1
$$

This wave function is solution of the following Schrödinger equation

$$
(\widetilde{\Sigma})\left\{\begin{array}{l}
i \frac{\partial \phi}{\partial \tau}(\tau, z)=-\frac{\partial^{2} \phi}{\partial z^{2}}(\tau, z), \tau \in \mathbb{R}_{+}^{*}, z \in(0, l(\tau)), \\
\phi(\tau, 0)=\phi(\tau, l(\tau))=0, \tau \in \mathbb{R}_{+}^{*}
\end{array}\right.
$$

The system $(\widetilde{\Sigma})$ is a control system in which

- the state is the wave function $\phi$, with (1.1) for every $\tau$;

- the control is the function $l$, with $l(0)=l\left(\tau_{f}\right)=1$, where $\tau_{f}$ is the final time.

In order to work on a more convenient control system, we perform changes of space variable $z \rightarrow x$, time variable $\tau \rightarrow t$, wave function $\phi(\tau, z) \rightarrow \psi(t, x)$, and control $l \rightarrow u$ which are presented in Section 1.3. They lead to the equivalent nonlinear control system

$$
(\Sigma)\left\{\begin{array}{l}
i \frac{\partial \psi}{\partial t}(t, x)=-\frac{\partial^{2} \psi}{\partial x^{2}}(t, x)+\left[\dot{u}(t)-4 u^{2}(t)\right] x^{2} \psi(t, x), t \in \mathbb{R}_{+}^{*}, x \in(0,1), \\
\psi(t, 0)=\psi(t, 1)=0, t \in \mathbb{R}_{+}^{*}
\end{array}\right.
$$

in which

- the state variable is the wave function $\psi$, with $\int_{0}^{1}|\psi(t, x)|^{2} \mathrm{~d} x=1$ for every $t$;

- the control is the real valued time depending function $u$, with $u(0)=u\left(t_{f}\right)=0, \int_{0}^{t_{f}} u(s) \mathrm{d} s=0$ where $t_{f}$ is the final time.

The system $(\Sigma)$ is easier to deal with than $(\widetilde{\Sigma})$ because it is posed on a fixed space domain.

Definition 1. Let $T_{1}<T_{2}$ be two real numbers and $u \in C^{1}\left(\left[T_{1}, T_{2}\right], \mathbb{R}\right)$. A function $\psi$ is a solution of $(\Sigma)$ if $\psi$ belongs to $C^{0}\left(\left[T_{1}, T_{2}\right], H^{2} \cap H_{0}^{1}((0,1), \mathbb{C})\right) \cap C^{1}\left(\left[T_{1}, T_{2}\right], L^{2}((0,1), \mathbb{C})\right)$ and satisfies the first equality of $(\Sigma)$ in $L^{2}((0,1), \mathbb{R})$, for every $t \in\left(T_{1}, T_{2}\right)$. Then, we say that $(\psi, u)$ is a trajectory of the control system $(\Sigma)$.

We give a sense to the solution of the initial problem $(\widetilde{\Sigma})$, posed on a variable domain, by using this definition of solution for the new system $(\Sigma)$ posed on a fixed domain : given a regular function $l:[0,+\infty) \rightarrow(0,+\infty)$ (regular enough so that the corresponding function $u$ is $C^{1}$ ), a function $\phi(\tau, z)$ is said to be a solution of $(\widetilde{\Sigma})$ if the corresponding function $\psi(t, x)$ through the changes $z \rightarrow x, \tau \rightarrow t, l \rightarrow u$ is a solution of $(\Sigma)$ in the sense of the previous definition.

Let us introduce the unitary $L^{2}((0,1), \mathbb{C})$-sphere $\mathcal{S}$ and the operator $A$ defined by

$$
D(A):=H^{2} \cap H_{0}^{1}((0,1), \mathbb{C}), \quad A \varphi:=-\varphi^{\prime \prime} .
$$

For every $n \in \mathbb{N}^{*}$,

$$
\varphi_{n}(x):=\sqrt{2} \sin (n \pi x)
$$

is an eigenvector of $A$ associated to the eigenvalue $\lambda_{n}:=(n \pi)^{2}$ and the family $\left(\varphi_{n}\right)_{n \in \mathbb{N}^{*}}$ is orthonormal in $L^{2}((0,1), \mathbb{C})$. For every $n \in \mathbb{N}^{*}$, the function

$$
\psi_{n}(t, x):=\varphi_{n}(x) \mathrm{e}^{-i \lambda_{n} t}
$$

is a solution of $(\Sigma)$ with $u \equiv 0$. For $s>0$, we introduce the space

$$
H_{(0)}^{s}((0,1), \mathbb{C}):=D\left(A^{s / 2}\right) .
$$


Since we will work with control functions $u$ with zero mean value, we introduce, for $s \geqslant 0$, the spaces

$$
\overline{H_{0}^{s}}((0, T), \mathbb{R}):=\left\{u \in H_{0}^{s}((0, T), \mathbb{R}) ; \int_{0}^{T} u(t) \mathrm{d} t=0\right\} .
$$

We write $\overline{L^{2}}((0, T), \mathbb{R})$ instead of $\overline{H^{0}}((0, T), \mathbb{R})$. The main result of this article is the following one.

Theorem 1. Let $\epsilon>0$. For every $n \in \mathbb{N}^{*}$, there exists $\eta_{n}>0$ such that, for every $n_{0}, n_{f} \in \mathbb{N}^{*}$, for every $\psi_{0}, \psi_{f} \in H_{(0)}^{5+\epsilon}((0,1), \mathbb{R}) \cap \mathcal{S}$ with

$$
\left\|\psi_{0}-\varphi_{n_{0}}\right\|_{H^{5+\epsilon}}<\eta_{n_{0}}, \quad\left\|\psi_{f}-\varphi_{n_{f}}\right\|_{H^{5+\epsilon}}<\eta_{n_{f}}
$$

there exists a time $\mathcal{T}$ and a trajectory $(\psi, u)$ of $(\Sigma)$ on $[0, \mathcal{T}]$ which satisfies $\psi(0)=\psi_{0}, \psi(\mathcal{T})=\psi_{f}$, and $u \in \overline{H_{0}^{2}}((0, \mathcal{T}), \mathbb{R})$.

Thus, we also have the following important corollary.

Corollary 1. For every $n_{0}, n_{f} \in \mathbb{N}^{*}$, there exists a time $\mathcal{T}$ and a trajectory $(\psi, u)$ of $(\Sigma)$ on $[0, \mathcal{T}]$ which satisfies $\psi(0)=\varphi_{n_{0}}, \psi(\mathcal{T})=\varphi_{n_{f}}$ and $u \in \overline{H_{0}^{2}}((0, \mathcal{T}), \mathbb{R})$.

Using the changes of variables presented in Section 1.3 this corresponds to the following controllability result for the initial system.

Theorem 2. For every $n_{0}, n_{f} \in \mathbb{N}^{*}$, there exists $\mathcal{T}>0$ and a trajectory $(\phi, l)$ of $(\widetilde{\Sigma})$ on $[0, \mathcal{T}]$ such that $l \in C^{2}\left([0, \mathcal{T}], \mathbb{R}_{+}^{*}\right), l(0)=l(\mathcal{T})=1, \phi(0)=\varphi_{n_{0}}, \phi(\mathcal{T})=\varphi_{n_{f}}$.

In Section 1.2, one mentions some other works about the controllability of Schrödinger equations using other methods.

In Section 1.3, one details the changes of variables and functions that transform $(\widetilde{\Sigma})$ into $(\Sigma)$.

In Section 1.4, one presents a previous non controllability result for $(\Sigma)$ and explain why this negative result can hold at the same time as the affirmative controllability result (Th. 1).

In Section 1.5, one gives a sketch of the proof: the global strategy is a compactness argument that needs local controllability results around many periodic trajectories. All those local results are proved thanks to the linearization principle for control problems. However the controllability of the linearized systems does not hold in suitable functional spaces, because of a loss of regularity, so, one cannot conclude with the inverse mapping theorem, and we use a Nash-Moser theorem. For some of those trajectories, the linearized system misses certain directions (it is controllable 'up to codimension one') and we exploit second order terms.

Sections 2-6 are dedicated to the different steps of the proof, announced in Section 1.5.

Finally, Section 7 gives some remarks and conjectures about this work.

\subsection{A brief literature review}

An good introduction to control questions for Schrödinger equations is [38].

First, the controllability of finite dimensional quantum systems (i.e. modeled by an ordinary differential equation) is well understood. Let us consider the quantum system

$$
i \frac{\mathrm{d} X}{\mathrm{~d} t}=H_{0} X+u(t) H_{1} X
$$

where $X \in \mathbb{C}^{n}$ is the state, $H_{0}, H_{1}$ are $n * n$ hermitian matrices, and $t \mapsto u(t) \in \mathbb{R}$ is the control. The controllability of (1.3) is linked to the rank of the Lie algebra spanned by $H_{0}$ and $H_{1}$ (see for instance [1,3,11]). Another interpretation of the controllability of (1.3) is the connectivity graph criterion [37].

In infinite dimension, there are cases where the iterated Lie brackets provide the right intuition. For instance, it holds for the harmonic oscillator [35]. However, the Lie brackets are often less powerful in infinite dimension 
than in finite dimension, thus, the exact controllability of an infinite dimensional bilinear system (i.e. modeled by a partial differential equation) is a more difficult problem.

Results on distributed and boundary exact controllability for linear Schrödinger equations are the subjects of $[31-34]$.

Optimal control techniques have been investigated for Schrödinger equations with a non linearity of Hartee type in [5,12] and [7]. An algorithm for the calculus of such optimal control is studied in [6].

Finally, non controllability results are proved in [37] and [28] for some particular linear and non linear Schrödinger equations. The result of [37] is discussed in Section 1.4.

\subsection{Changes of time variable, space variable and wave function}

In order to get a problem posed on a fixed domain, we consider the change of space variable and function

$$
\begin{aligned}
& x:=\frac{1}{l(t)} z, \\
& \zeta(\tau, x):=\phi(\tau, z) .
\end{aligned}
$$

We get the following system

$$
\left\{\begin{array}{l}
i \frac{\partial \zeta}{\partial \tau}(\tau, x)=-\frac{1}{l(\tau)^{2}} \frac{\partial^{2} \zeta}{\partial x^{2}}(\tau, x)+i \frac{i(\tau)}{l(\tau)} x \frac{\partial \zeta}{\partial x}(\tau, x), \tau \in \mathbb{R}_{+}, x \in(0,1) \\
\zeta(\tau, 0)=\zeta(\tau, 1)=0
\end{array}\right.
$$

In order to make disappear the term before the Laplacian, we consider the change of time variable defined by

$$
\begin{aligned}
& t:=\int_{0}^{\tau} \frac{1}{l(\sigma)^{2}} \mathrm{~d} \sigma, \\
& \xi(t, x):=\zeta(\tau, x),
\end{aligned}
$$

which gives

$$
\left\{\begin{array}{l}
i \frac{\partial \xi}{\partial t}(t, x)=-\frac{\partial^{2} \xi}{\partial x^{2}}(t, x)+i 4 u(t) x \frac{\partial \xi}{\partial x}(t, x), t \in \mathbb{R}_{+}, x \in(0,1), \\
\xi(t, 0)=\xi(t, 1)=0
\end{array}\right.
$$

where $u(t):=\frac{1}{4} \dot{l}(\tau) l(\tau)$, which is equivalent to

$$
l(\tau)=\exp \left(4 \int_{0}^{t} u(s) \mathrm{d} s\right)
$$

Now the change of wave function

$$
\psi(t, x):=\xi(t, x) \mathrm{e}^{-i u(t) x^{2}+2 \int_{0}^{t} u(s) \mathrm{d} s}
$$

leads to the system $(\Sigma)$. In order to justify that the controllability of $(\Sigma)$ gives the controllability of $(\widetilde{\Sigma})$, we need to prove that the map $l \mapsto u$ is surjective. For the control problem on $(\widetilde{\Sigma})$ to have a sense, we look for $l:\left[0, \tau_{f}\right] \rightarrow \mathbb{R}_{+}^{*}$ continuous with $l(0)=l\left(\tau_{f}\right)=1$, which, together with (1.4) implies $\int_{0}^{t_{f}} u(s) d s=0$, where $\tau_{f}$ and $t_{f}$ are linked through the relation

$$
t_{f}=\int_{0}^{\tau_{f}} \frac{1}{l(\tau)^{2}} \mathrm{~d} \tau
$$

In order to have $\psi(0)=\phi(0)$ and $\psi\left(t_{f}\right)=\phi\left(\tau_{f}\right)$, we look for $u$ such that $u(0)=u\left(t_{f}\right)=0$. In the proof of Theorem 1 , we will get the time $\mathcal{T}$ and the control $u \in \overline{H_{0}^{2}}((0, \mathcal{T}), \mathbb{R})$ in the following way

$$
\begin{aligned}
& \mathcal{T}=m T \\
& u(t)=u_{k}(t-k T) \quad \text { for every } \quad t \in[k T,(k+1) T] \quad \text { and for every } \quad k \in\{0, \ldots, m-1\},
\end{aligned}
$$


where $m$ is a positive integer, $T:=2 / \pi, u_{k} \in \overline{H_{0}^{2}}((0, T), \mathbb{R})$ is small. Thus, the following proposition is sufficient. Proposition 1. Let $T>0$ and $\epsilon \in(0,1)$. Let $u \in C^{0}([0, T], \mathbb{R})$ be such that

$$
u(0)=u(T)=\int_{0}^{T} u(s) \mathrm{d} s=0,
$$

small in the following sense

$$
\begin{gathered}
4\|u\|_{L^{1}(0, T)} \exp \left(4\|u\|_{L^{1}(0, T)}\right)<\epsilon \\
\frac{8 T(1+\epsilon)}{(1-\epsilon)^{4}}\|u\|_{L^{\infty}(0, T)} \exp \left(4\|u\|_{L^{1}(0, T)}\right)<1 .
\end{gathered}
$$

We define $u$ on $\mathbb{R}_{+}$by $u \equiv 0$ on $[T,+\infty]$. Then, there exists a unique $l \in C^{0}\left(\mathbb{R}_{+},[1-\epsilon, 1+\epsilon]\right)$ solution of

$$
l(\tau)=\exp \left(4 \int_{0}^{t(\tau)} u(s) \mathrm{d} s\right) \text { where } t(\tau):=\int_{0}^{\tau} \frac{1}{l(\sigma)^{2}} \mathrm{~d} \sigma .
$$

Proof. The space

$$
V_{\epsilon}:=\left\{l \in C^{0}\left(\mathbb{R}_{+},[1-\epsilon, 1+\epsilon]\right) ; l(0)=1 \quad \text { and } \quad l \equiv 1 \quad \text { on } \quad\left[T(1+\epsilon)^{2},+\infty\right)\right\}
$$

is complete for the $L^{\infty}\left(\mathbb{R}_{+}, \mathbb{R}\right)$-norm. For $l \in V_{\epsilon}$, we define $\Phi(l): \mathbb{R}_{+} \rightarrow \mathbb{R}, \Phi(l)(\tau):=\exp \left(4 \int_{0}^{t(\tau)} u(s) \mathrm{d} s\right)$. Assumption (1.6) justifies that $\Phi$ maps $V_{\epsilon}$ into itself, and assumption (1.7) justifies that $\Phi$ is a contraction. We conclude thanks to the Banach fixed point theorem.

\subsection{A previous non controllability result}

In [4], Ball, Marsden and Slemrod discuss the controllability of infinite dimensional bilinear control systems of the form

$$
\dot{w}(t)=\mathcal{A} w(t)+p(t) \mathcal{B}(w(t))
$$

where the state is $w$ and the control is $p$. Thanks to Baire lemma, they prove the following non controllability result.

Theorem 3. Let $X$ be a Banach space with $\operatorname{dim}(X)=+\infty$. Let $\mathcal{A}$ generate a $C^{0}$-semi group of bounded linear operators on $X$ and $\mathcal{B}: X \rightarrow X$ be a bounded linear operator. Let $w_{0} \in X$ be fixed and let $w\left(t ; p, w_{0}\right)$ denote the unique solution of $(1.8)$ for $p \in L_{l o c}^{1}((0,+\infty), \mathbb{R})$ with $w(0)=w_{0}$. The set of states accessible from $w_{0}$ defined by

$$
S\left(w_{0}\right):=\left\{w\left(t ; p, w_{0}\right) ; t \geqslant 0, p \in L_{l o c}^{r}((0, \infty), \mathbb{R}), r>1\right\}
$$

is contained in a countable union of compact subsets of $X$ and, in particular, has dense complement.

As noticed by Turinici in [37], Theorem 3 shows that, for the bilinear control system

$$
\left\{\begin{array}{l}
i \dot{\psi}=-\psi^{\prime \prime}+p(t) x^{2} \psi, x \in(0,1), \\
\psi(t, 0)=\psi(t, 1)=0
\end{array}\right.
$$

given $\psi_{0} \in X:=\mathcal{S} \cap H_{(0)}^{2}((0,1), \mathbb{C})$, the set of $\psi(t)$ in $X$ accessible from the initial condition $\psi_{0}$, by using controls in $p \in L_{\text {loc }}^{r}((0, \infty), \mathbb{R}), r>1$, has dense complement in $X$. Thus, the system $(\Sigma)$ is not controllable in $\mathcal{S} \cap H_{(0)}^{2}((0,1), \mathbb{C})$, with control functions $u$ in $H_{0}^{1}((0, T), \mathbb{R}), T>0$.

However, there is no obstruction for having controllability in other spaces. For example, Theorem 3 does not apply with

$$
\widetilde{X}:=H_{(0)}^{3}((0,1), \mathbb{C})
$$


instead of $X$ because the operator $\mathcal{B}$, defined by $\mathcal{B} \varphi:=x^{2} \varphi$, does not map $\widetilde{X}$ into $\widetilde{X}$.

In this article, we prove local controllability results in $H_{(0)}^{5+\epsilon}((0,1), \mathbb{C})$, with $\epsilon>0$ and with control functions $u$ in $\overline{H_{0}^{2}}((0, T), \mathbb{R})$ with $T=2 / \pi$. Thus, the negative result proved by G. Turinici relies on a choice of functional spaces which does not allow controllability. In order to state affirmative controllability results, one must

- either control $\psi$ in $H_{(0)}^{2}((0,1), \mathbb{C})$ but with a control functions set larger than $\overline{H_{0}^{1}}((0, T), \mathbb{R})$, for example $\overline{L^{2}}((0, T), \mathbb{R})$

- or control $\psi$ using the control functions set $\overline{H_{0}^{1}}((0, T), \mathbb{R})$, but in a smaller space than $H_{(0)}^{2}((0,1), \mathbb{C})$, for example $H_{(0)}^{3}((0,1), \mathbb{C})$.

In the regularity assumption $H^{5+\epsilon}((0,1), \mathbb{R})$, the term $+\epsilon$ is probably only technical. We conjecture that $(\Sigma)$ is controllable

- in $H_{(0)}^{3}((0,1), \mathbb{C})$ with control functions $u$ in $\overline{H_{0}^{1}}((0, T), \mathbb{R})$;

- in $H_{(0)}^{5}((0,1), \mathbb{C})$ with control functions $u$ in $\overline{H_{0}^{2}}((0, T), \mathbb{R})$;

- in $H_{(0)}^{7}((0,1), \mathbb{C})$ with control functions $u$ in $\overline{H_{0}^{3}}((0, T), \mathbb{R})$, etc.

Because it is the case for the linearized systems studied in Section 2. This conjectures are open problems.

\subsection{Sketch of the proof}

The technic used in this proof are very close to the one used in [10]. We extend the use of the Nash-Moser theorem to a nonlinear control system which is not bilinear.

\subsubsection{Global strategy: compactness argument}

Thanks to the reversibility of the control system $(\Sigma)$, in order to get Theorem 1 , it is sufficient to prove it with $n_{f}=n_{0}+1$. We prove it with $n_{0}=1$ and $n_{f}=2$ to simplify.

First, we prove the local controllability of $(\Sigma)$ in $H^{5+\epsilon}((0,1), \mathbb{C})$, in time $T=2 / \pi$ or $4 / \pi$ around the trajectories

$$
\left(\sqrt{1-\theta_{2}-\theta_{3}} \psi_{1}+\sqrt{\theta_{2}} \psi_{2}+\sqrt{\theta_{3}} \psi_{3}, u \equiv 0\right),
$$

for every $\left(\theta_{2}, \theta_{3}\right) \in \mathcal{D}:=\left\{(x, y) \in(0,1)^{2}, 0<x+y<1\right\} \cup\{(0,0),(1,0)\}$. Then, we know that, for every $\left(\theta_{2}, \theta_{3}\right) \in$ $\mathcal{D}$, there exists a nonempty open $H^{5+\epsilon}((0,1), \mathbb{C})$-ball $B_{\left(\theta_{2}, \theta_{3}\right)}$ centered at $\left(\sqrt{1-\theta_{2}-\theta_{3}} \psi_{1}+\sqrt{\theta_{2}} \psi_{2}+\sqrt{\theta_{3}} \psi_{3}\right)(0)$ such that $(\Sigma)$ can be moved in finite time between any two points in $B_{\left(\theta_{2}, \theta_{3}\right)}$.

We conclude thanks to a compactness argument. Let $f \in C^{0}([0,1], \mathbb{R})$ be such that

$$
f(0)=f(1)=0,0 \leqslant f(x) \text { and } 0 \leqslant x+f(x) \leqslant 1 \text { for every } x \in[0,1] .
$$

The curve

$$
\left[\varphi_{1}, \varphi_{2}\right]:=\left\{\sqrt{1-\theta-f(\theta)} \varphi_{1}+\sqrt{\theta} \varphi_{2}+\sqrt{f(\theta)} \varphi_{3} ; \theta \in[0,1]\right\},
$$

is compact in $H^{5+\epsilon}((0,1), \mathbb{R})$ and covered by $\cup_{0 \leqslant \theta \leqslant 1} B_{(\theta, f(\theta))}$ thus, there exists an increasing finite family $\left(\theta_{n}\right)_{0 \leqslant n \leqslant N}$ such that $\left[\varphi_{1}, \varphi_{2}\right]$ is covered by $\cup_{0 \leqslant n \leqslant N} B_{n}$ with $B_{n}:=B_{\left(\theta_{n}, f\left(\theta_{n}\right)\right)}$. We can assume $B_{n} \cap B_{n+1} \neq \emptyset$ for $n=0, \ldots, N-1, B_{0}=B_{(0,0)}$ and $B_{N}=B_{(1,0)}$. Given $\psi_{0} \in B_{0}$ and $\psi_{f} \in B_{N}$ we can move $(\Sigma)$ from $\psi_{0}$ to $\psi_{f}$ in finite time in the following way:

- we move from $\psi_{0}$ to some point $\xi_{1} \in B_{0} \cap B_{1}$ in finite time;

- we move from $\xi_{1}$ to some point $\xi_{2} \in B_{1} \cap B_{2}$, etc.

Remark 1. It would be more natural to use the path

$$
\left[\varphi_{1}, \varphi_{2}\right]:=\left\{\sqrt{1-\theta} \varphi_{1}+\sqrt{\theta} \varphi_{2} ; \theta \in[0,1]\right\}
$$


in the compactness argument, as in [10]. We chose the path (1.10) because the local controllability of $(\Sigma)$ is easier to be proved around $\sqrt{1-\theta_{2}-\theta_{3}} \psi_{1}+\sqrt{\theta_{2}} \psi_{2}+\sqrt{\theta_{3}} \psi_{3}$ for $\left(\theta_{2}, \theta_{3}\right) \in \operatorname{Int}(\mathcal{D})$, than around $\sqrt{1-\theta} \psi_{1}+\sqrt{\theta_{2}} \psi_{2}$ for $\theta \in(0,1)$. We detail this additional difficulty in Remark 3 However, the path (1.11) could also be used to prove Theorem 1, one proposes an adaptation of the present proof in Remark 3 in order to do so.

Now, let us explain the proof of the local controllability of $(\Sigma)$ around

$$
\sqrt{1-\theta_{2}-\theta_{3}} \psi_{1}+\sqrt{\theta_{2}} \psi_{2}+\sqrt{\theta_{3}} \psi_{3}
$$

for $\left(\theta_{2}, \theta_{3}\right) \in \mathcal{D}$. We explain in the next section that the strategy can be the same for every $\left(\theta_{2}, \theta_{3}\right) \in \operatorname{Int}(\mathcal{D})$ but has to be different for $\left(\theta_{2}, \theta_{3}\right) \in\{(0,0),(1,0)\}$.

\subsubsection{Different behaviors for the linearized systems}

In order to get the local controllability of a nonlinear control system around some trajectory, a classical approach is the following one :

- first, we prove the controllability of the linearized system around this trajectory;

- then we conclude applying the inverse mapping theorem to the end-point map $\Theta$ defined by

$$
\Theta:\left(\psi_{0}, u\right) \mapsto(\psi(0), \psi(T)),
$$

where $\psi$ is the solution of $(\Sigma)$ with control $u$ and initial condition $\psi(0)=\psi_{0}$.

Thus, it is natural to start with the study of the linearized system of $(\Sigma)$ around the trajectories

$$
\left(\sqrt{1-\theta_{2}-\theta_{3}} \psi_{1}+\sqrt{\theta_{2}} \psi_{2}+\sqrt{\theta_{3}} \psi_{3}, u \equiv 0\right)
$$

for $\left(\theta_{2}, \theta_{3}\right) \in \mathcal{D}$, which is

$$
\left(\Sigma_{\theta_{2}, \theta_{3}}\right)\left\{\begin{array}{l}
i \frac{\partial \Psi}{\partial t}(t, x)=-\frac{\partial^{2} \Psi}{\partial x^{2}}(t, x)+\dot{v}(t) x^{2}\left(\sqrt{1-\theta_{2}-\theta_{3}} \psi_{1}+\sqrt{\theta_{2}} \psi_{2}+\sqrt{\theta_{3}} \psi_{3}\right)(t, x), t \in \mathbb{R}_{+}, x \in(0,1), \\
\Psi(t, 0)=\Psi(t, 1)=0 .
\end{array}\right.
$$

For $z \in \mathbb{C}, \Re(z)$ (resp. $\Im(z)$ ) denotes the real (resp. imaginary) part of $z$. For every point $\xi$ in the $L^{2}((0,1), \mathbb{C})$ sphere $\mathcal{S}, T_{\mathcal{S}}(\xi)$ denotes the tangent space to $\mathcal{S}$ at the point $\xi$,

$$
T_{\mathcal{S}}(\xi):=\left\{\varphi \in L^{2}((0,1), \mathbb{C}) ; \Re\left(\int_{0}^{1} \xi(x) \overline{\varphi(x)} \mathrm{d} x\right)=0\right\}
$$

The system $\left(\Sigma_{\theta_{2}, \theta_{3}}\right)$ is a control system in which

- the state is the function $\Psi$, with $\Psi(t) \in T_{\mathcal{S}}\left[\left(\sqrt{1-\theta_{2}-\theta_{3}} \psi_{1}+\sqrt{\theta_{2}} \psi_{2}+\sqrt{\theta_{3}} \psi_{3}\right)(t)\right]$, for every $t$;

- the control is the real valued time depending function $v$, with $v(0)=v(T)=\int_{0}^{t_{f}} v(s) \mathrm{d} s=0$.

In Section 2, we prove the following result.

Theorem 4. Let $\left(\theta_{2}, \theta_{3}\right) \in \operatorname{Int}(\mathcal{D})$ and $T>2 /(3 \pi)$. The system $\left(\Sigma_{\theta_{2}, \theta_{3}}\right)$ is controllable in time $T$ : for every $\Psi_{0}, \Psi_{f} \in H_{(0)}^{3}((0,1), \mathbb{C})$ with

$$
\begin{aligned}
& \Psi_{0} \in T_{\mathcal{S}}\left[\left(\sqrt{1-\theta_{2}-\theta_{3}} \psi_{1}+\sqrt{\theta_{2}} \psi_{2}+\sqrt{\theta_{3}} \psi_{3}\right)(0)\right], \\
& \Psi_{f} \in T_{\mathcal{S}}\left[\left(\sqrt{1-\theta_{2}-\theta_{3}} \psi_{1}+\sqrt{\theta_{2}} \psi_{2}+\sqrt{\theta_{3}} \psi_{3}\right)(T)\right],
\end{aligned}
$$

there exists a trajectory $(\Psi, v)$ of $\left(\Sigma_{\theta_{2}, \theta_{3}}\right)$ with $\Psi(0)=\Psi_{0}, \Psi(T)=\Psi_{f}, v \in \overline{H_{0}^{1}}((0, T), \mathbb{R})$. 
The system $\left(\Sigma_{0,0}\right)$ is not controllable : for every $T>0$ and for every $v \in \overline{H_{0}^{1}}((0, T), \mathbb{R})$, the solution $\Psi$ of $\left(\Sigma_{0,0}\right)$ satisfies

Let $T>0, \Psi_{0}, \Psi_{f} \in H_{(0)}^{3}((0,1), \mathbb{C})$ with

$$
\left\langle\Psi(T), \varphi_{1}\right\rangle=\left\langle\Psi(0), \varphi_{1}\right\rangle \mathrm{e}^{-i \lambda_{1} T}
$$

$$
\Psi_{0} \in T_{\mathcal{S}}\left(\psi_{1}(0)\right), \quad \Psi_{f} \in T_{\mathcal{S}}\left(\psi_{1}(T)\right) \quad \text { and } \quad \Im\left(\left\langle\Psi_{f}, \varphi_{1}\right\rangle\right)=\Im\left(\left\langle\Psi_{0}, \varphi_{1}\right\rangle \mathrm{e}^{-i \lambda_{1} T}\right)
$$

There exists a trajectory $(\Psi, v)$ of $\left(\Sigma_{0,0}\right)$ with $\Psi(0)=\Psi_{0}, \Psi(T)=\Psi_{f}, v \in \overline{H_{0}^{1}}((0, T), \mathbb{R})$.

For the linear system $\left(\Sigma_{0,0}\right)$, we can control all the components $\left\langle\Psi(t), \varphi_{k}\right\rangle$ for $k \geqslant 2$ and we cannot control $\Im\left\langle\Psi(t), \psi_{1}(t)\right\rangle$. We call this situation controllability up to codimension one, as in [10]. For the linearized system $\left(\Sigma_{1,0}\right)$ around $\left(\psi_{2}, u \equiv 0\right)$, we can control all the components $\left\langle\psi(t), \varphi_{k}\right\rangle$ for $k \in \mathbb{N}^{*}, k \neq 2$, and we cannot control $\Im\left\langle\Psi(t), \psi_{2}(t)\right\rangle$.

1.5.3. Local controllability around $\sqrt{1-\theta_{2}-\theta_{3}} \psi_{1}+\sqrt{\theta_{2}} \psi_{2}+\sqrt{\theta_{3}} \psi_{3}$ for $\left(\theta_{2}, \theta_{3}\right) \in \operatorname{Int}(\mathcal{D})$.

The goal of Section 4 , is the proof of the following result.

Theorem 5. Let $\left(\theta_{2}, \theta_{3}\right) \in \operatorname{Int}(\mathcal{D}), T:=2 / \pi$ and $\epsilon$ be an arbitrary positive real number. There exist $C>0$ and a neighborhood $V_{0}$ (resp. $\left.V_{f}\right)$ of $\left(\sqrt{1-\theta_{2}-\theta_{3}} \psi_{1}+\sqrt{\theta_{2}} \psi_{2}+\sqrt{\theta_{3}} \psi_{3}\right)(0)$ (resp. $\left(\sqrt{1-\theta_{2}-\theta_{3}} \psi_{1}+\sqrt{\theta_{2}} \psi_{2}+\right.$ $\left.\left.\sqrt{\theta_{3}} \psi_{3}\right)(T)\right)$ in $\mathcal{S} \cap H_{(0)}^{5+\epsilon}((0,1), \mathbb{C})$ such that, for every $\psi_{0} \in V_{0}, \psi_{f} \in V_{f}$, there exists a trajectory $(\psi, u)$ of $(\Sigma)$ with $\psi(0)=\psi_{0}, \psi(T)=\psi_{f}, u \in \overline{H_{0}^{2}}((0, T), \mathbb{R})$, moreover

$$
\begin{aligned}
\|u\|_{\overline{H_{0}^{2}}((0, T), \mathbb{R})} \leqslant \quad & C\left[\left\|\psi_{0}-\left(\sqrt{1-\theta_{2}-\theta_{3}} \psi_{1}+\sqrt{\theta_{2}} \psi_{2}+\sqrt{\theta_{3}} \psi_{3}\right)(0)\right\|_{H^{5+\epsilon}}\right. \\
& \left.+\left\|\psi_{f}-\left(\sqrt{1-\theta_{2}-\theta_{3}} \psi_{1}+\sqrt{\theta_{2}} \psi_{2}+\sqrt{\theta_{3}} \psi_{3}\right)(T)\right\|_{H^{5+\epsilon}}\right] .
\end{aligned}
$$

Remark 2. Theorem 5 is written with $T=2 / \pi$ because, in this case, $\left(\sqrt{1-\theta_{2}-\theta_{3}} \psi_{1}+\sqrt{\theta_{2}} \psi_{2}+\sqrt{\theta_{3}} \psi_{3}\right)(0)=$ $\left(\sqrt{1-\theta_{2}-\theta_{3}} \psi_{1}+\sqrt{\theta_{2}} \psi_{2}+\sqrt{\theta_{3}} \psi_{3}\right)(T)$, and this condition is needed in the compactness argument (see Sect. 1.5.1). However, this theorem may hold with other values of $T$. This is discussed in Section 7.2.

The first part of Theorem 4 is not sufficient to conclude the local controllability of $(\Sigma)$ around $\sqrt{1-\theta_{2}-\theta_{3}} \psi_{1}+$ $\sqrt{\theta_{2}} \psi_{2}+\sqrt{\theta_{3}} \psi_{3}$ for $\left(\theta_{2}, \theta_{3}\right) \in \operatorname{Int}(\mathcal{D})$, by applying the classical inverse mapping theorem. Indeed, the end point map $\Phi$ is well defined and of class $C^{1}$ between the following spaces

$$
\begin{aligned}
& \Phi:\left[\mathcal{S} \cap H_{(0)}^{2}((0,1), \mathbb{C})\right] \times \overline{H_{0}^{1}}((0, T), \mathbb{R}) \rightarrow\left[\mathcal{S} \cap H_{(0)}^{2}((0,1), \mathbb{C})\right] \times\left[\mathcal{S} \cap H_{(0)}^{2}((0,1), \mathbb{C})\right], \\
& \Phi:\left[\mathcal{S} \cap H_{(0)}^{3}((0,1), \mathbb{C})\right] \times \overline{H_{0}^{2}}((0, T), \mathbb{R}) \rightarrow\left[\mathcal{S} \cap H_{(0)}^{3}((0,1), \mathbb{C})\right] \times\left[\mathcal{S} \cap H_{(0)}^{3}((0,1), \mathbb{C})\right] .
\end{aligned}
$$

In order to apply the inverse mapping theorem to the map $\Phi$, we need to control the linearized system around $\left(\sqrt{1-\theta_{2}-\theta_{3}} \psi_{1}+\sqrt{\theta_{2}} \psi_{2}+\sqrt{\theta_{3}} \psi_{3}, u \equiv 0\right)$

- either in $H_{(0)}^{2}((0,1), \mathbb{C})$ with control functions in $\overline{H_{0}^{1}}((0, T), \mathbb{R})$;

- or in $H_{(0)}^{3}((0,1), \mathbb{C})$ with control functions in $\overline{H_{0}^{2}}((0, T), \mathbb{R})$,

but it is not possible (see Prop. 2). Theorem 4 provides a right inverse $\mathrm{d} \Phi\left(\sqrt{1-\theta_{2}-\theta_{3}} \varphi_{1}+\sqrt{\theta_{2}} \varphi_{2}+\sqrt{\theta_{3}} \varphi_{3}, 0\right)^{-1}$ defined between the following spaces

$$
H_{(0)}^{3}((0,1), \mathbb{C}) \times H_{(0)}^{3}((0,1), \mathbb{C}) \rightarrow H_{(0)}^{3}((0,1), \mathbb{C}) \times \overline{H_{0}^{1}}((0, T), \mathbb{R})
$$

We lose regularity in the controllability of the linearized system: the control function cannot be regular enough to apply the classical inverse mapping theorem.

We prove Theorem 5 by applying a Nash-Moser theorem stated in Section 3, and inspired from [26]. A similar version of this theorem is used in [8-10]. In Section 4.1, we give the context for the application of this theorem. In Sections 4.2, 4.3 and 4.4, we check its assumptions. 


\subsubsection{Local controllability around $\psi_{1}$ : expansion to the second order}

The goal of Section 5 is the proof of the following result.

Theorem 6. Let $T:=2 / \pi$ and $\epsilon>0$. There exist $C>0$ and a neighborhood $V_{0}$ (resp. $\left.V_{f}\right)$ of $\psi_{1}(0)($ resp. $\left.\psi_{1}(2 T)\right)$ in $\mathcal{S} \cap H_{(0)}^{5+\epsilon}((0,1), \mathbb{C})$ such that, for every $\psi_{0} \in V_{0}, \psi_{f} \in V_{f}$, there exists a trajectory $(\psi, u)$ of $(\Sigma)$ with $\psi(0)=\psi_{0}, \psi(2 T)=\psi_{f}, u \in \overline{H_{0}^{2}}((0,2 T), \mathbb{R})$, moreover

$$
\|u\|_{\overline{H_{0}^{2}}((0,2 T), \mathbb{R})} \leqslant C\left[\left\|\psi_{0}-\psi_{1}(0)\right\|_{H^{5+\epsilon}}+\left\|\psi_{f}-\psi_{1}(2 T)\right\|_{H^{5+\epsilon}}\right] .
$$

Again, for $T=2 / \pi$, we have $\psi_{1}(0)=\psi_{1}(2 T)=\varphi_{1}$, but this theorem is written in this way in order to discuss its generalization with $T \neq 2 / \pi$ in Section 7.2. The same result holds with everywhere $\psi_{2}$ instead of $\psi_{1}$.

Our strategy is in two steps. First, in Section 5.1, we state a local controllability up to codimension one result of $(\Sigma)$ around $\psi_{1}$. Then, in Section 5.2 , we justify that the second order term $\mathrm{d}^{2} \Phi\left(\varphi_{1}, 0\right)$ allows to move in the two directions $\pm i \psi_{1}(T)$ which are missed by the linearized system. Finally, in Section 5.3 we prove Theorem 6 , thanks to the intermediate value theorem.

These techniques have already been used by Coron and Crépeau in [18]. In their situation, the second order term was not sufficient to conclude, they used the third order term.

The local controllability up to codimension one of $(\Sigma)$ stated in Section 5.1 is proved in Section 6 by applying a Nash-Moser theorem stated in Section 3.

Remark 3. It would be more natural to use the path

$$
\left[\varphi_{1}, \varphi_{2}\right]:=\left\{\sqrt{1-\theta} \varphi_{1}+\sqrt{\theta} \varphi_{2}, \theta \in[0,1]\right\}
$$

in the compactness argument presented in Section 1.5.1. However, for $\theta \in(0,1)$, the linearized system of $(\Sigma)$ around $\left(\sqrt{1-\theta} \psi_{1}+\sqrt{\theta} \psi_{2}, u \equiv 0\right)$ is not controllable: as in the case $\theta \in\{0,1\}$, it misses two directions. Expansions to the second order would probably also give the local controllability in $H^{5+\epsilon}((0,1), \mathbb{C})$ of $(\Sigma)$ around $\sqrt{1-\theta} \psi_{1}+\sqrt{\theta} \psi_{2}$ for $\theta \in(0,1)$, with control functions $u \in \overline{H_{0}^{2}}((0,4 / \pi), \mathbb{R})$.

\section{Controllability of the linear system $\left(\Sigma_{\theta_{2}, \theta_{3}}\right)$}

The goal of this section is the proof of Theorem 4 .

Let $\left(\theta_{2}, \theta_{3}\right) \in \mathcal{D}, T>0$ and $\Psi$ be a solution of $\left(\Sigma_{\theta_{2}, \theta_{3}}\right)$ for some $v \in \overline{H_{0}^{1}}((0, T), \mathbb{R})$ with $\Psi(0)=0$. For every $t \in[0, T]$, we have

$$
\Psi(t)=\sum_{k=1}^{\infty} y_{k}(t) \varphi_{k} \quad \text { where } \quad y_{k}(t):=\left\langle\Psi(t), \varphi_{k}\right\rangle
$$

and $\langle.,$.$\rangle denotes the scalar product on L^{2}((0,1), \mathbb{C})$. The partial differential equation satisfied by $\Psi$ provides, for every $k \in \mathbb{N}^{*}$, the following expression

$$
y_{k}(t)=-i \int_{0}^{t} \dot{v}(\tau)\left(\sqrt{1-\theta_{2}-\theta_{3}} a_{k} \mathrm{e}^{i\left(\lambda_{k}-\lambda_{1}\right) \tau}+\sqrt{\theta_{2}} b_{k} \mathrm{e}^{i\left(\lambda_{k}-\lambda_{2}\right) \tau}+\sqrt{\theta_{3}} c_{k} \mathrm{e}^{i\left(\lambda_{k}-\lambda_{3}\right) \tau}\right) \mathrm{d} \tau \mathrm{e}^{-i \lambda_{k} t}
$$

where

Let $\Psi_{f} \in H_{(0)}^{2}((0,1), \mathbb{C})$ be such that

$$
a_{k}:=\left\langle x^{2} \varphi_{1}, \varphi_{k}\right\rangle, \quad b_{k}:=\left\langle x^{2} \varphi_{2}, \varphi_{k}\right\rangle, \quad c_{k}:=\left\langle x^{2} \varphi_{3}, \varphi_{k}\right\rangle .
$$

$$
\Psi_{f} \in T_{\mathcal{S}}\left[\left(\sqrt{1-\theta_{2}-\theta_{3}} \psi_{1}+\sqrt{\theta_{2}} \psi_{2}+\sqrt{\theta_{3}} \psi_{3}\right)(T)\right]
$$


The equality $\Psi(T)=\Psi_{f}$ is equivalent to: for every $k \in \mathbb{N}^{*}$,

$$
\begin{gathered}
\sqrt{1-\theta_{2}-\theta_{3}} a_{k} \int_{0}^{T} \dot{v}(\tau) \mathrm{e}^{i\left(\lambda_{k}-\lambda_{1}\right) \tau} \mathrm{d} \tau+ \\
=\sqrt{\theta_{2}} b_{k} \int_{0}^{T} \dot{v}(\tau) \mathrm{e}^{i\left(\lambda_{k}-\lambda_{2}\right) \tau} \mathrm{d} \tau+\sqrt{\theta_{3}} c_{k} \int_{0}^{T} \dot{v}(\tau) \mathrm{e}^{i\left(\lambda_{k}-\lambda_{3}\right) \tau} \mathrm{d} \tau \\
=i\left\langle\Psi_{f}, \varphi_{k}\right\rangle \mathrm{e}^{i \lambda_{k} T}
\end{gathered}
$$

The explicit expression (1.2) provides, for every $k, j \in \mathbb{N}^{*}$,

$$
\left\langle x^{2} \varphi_{j}, \varphi_{k}\right\rangle=\left\{\begin{array}{l}
\frac{(-1)^{k+j} 8 k j}{\pi^{2}(k+j)^{2}(k-j)^{2}}, \text { when } k \neq j \\
\frac{1}{3}-\frac{1}{2(k \pi)^{2}}, \text { when } k=j .
\end{array}\right.
$$

In particular, for every $k, j \in \mathbb{N}^{*},\left\langle x^{2} \varphi_{j}, \varphi_{k}\right\rangle \neq 0$.

Let $\left(\theta_{2}, \theta_{3}\right)=(0,0)$. The relation $(2.3)$ gives the following trigonometric moment problem

$$
\int_{0}^{T} \dot{v}(t) \mathrm{e}^{i\left(\lambda_{k}-\lambda_{1}\right) t} \mathrm{~d} t=\frac{i}{\left\langle x^{2} \varphi_{1}, \varphi_{k}\right\rangle}\left\langle\Psi_{f}, \varphi_{k}\right\rangle \mathrm{e}^{i \lambda_{k} T}, \text { for every } k \in \mathbb{N}^{*} .
$$

A necessary condition for the existence of a solution $v \in \overline{H_{0}^{1}}((0, T), \mathbb{R})$ is $\left\langle\Psi_{f}, \varphi_{1}\right\rangle=0$. Under this assumption, this moment problem has a solution $v \in \overline{H_{0}^{1}}((0, T), \mathbb{R})$ for every $T>0$, as soon as the right hand side of $(2.5)$ belongs to $l^{2}\left(\mathbb{N}^{*}, \mathbb{C}\right)$ (see [30], Th. 1.2.18), which is the case when $\Psi_{f} \in H_{(0)}^{3}((0,1), \mathbb{C})$.

The case $\left(\theta_{2}, \theta_{3}\right)=(1,0)$ can be treated in the same way.

Now, let us assume $\left(\theta_{2}, \theta_{3}\right) \in \operatorname{Int}(\mathcal{D})$. The relation (2.3) is satisfied, for instance, when

$$
\left\{\begin{aligned}
\int_{0}^{T} \dot{v}(t) \mathrm{e}^{i\left(\lambda_{2}-\lambda_{1}\right) t} \mathrm{~d} t & =\frac{1}{a_{2} \sqrt{1-\theta_{2}-\theta_{3}}}\left(i\left\langle\Psi_{f}, \psi_{2}(T)\right\rangle-\sqrt{\theta_{3}} b_{3} \bar{C}\right) \\
\int_{0}^{T} \dot{v}(t) \mathrm{e}^{i\left(\lambda_{3}-\lambda_{1}\right) t} \mathrm{~d} t & =\frac{1}{a_{3} \sqrt{1-\theta_{2}-\theta_{3}}}\left(i\left\langle\Psi_{f}, \psi_{3}(T)\right\rangle-\sqrt{\theta_{2}} b_{3} \bar{C}\right) \\
\int_{0}^{T} \dot{v}(t) \mathrm{e}^{i\left(\lambda_{3}-\lambda_{2}\right) t} \mathrm{~d} t & =C \\
\int_{0}^{T} \dot{v}(t) \mathrm{e}^{i\left(\lambda_{k}-\lambda_{1}\right) t} \mathrm{~d} t & =\frac{i \sqrt{1-\theta_{2}-\theta_{3}}}{a_{k}}\left\langle\Psi_{f}, \psi_{k}(T)\right\rangle, \forall k \geqslant 4, \\
\int_{0}^{T} \dot{v}(t) \mathrm{e}^{i\left(\lambda_{k}-\lambda_{2}\right) t} \mathrm{~d} t & =\frac{i \sqrt{\theta_{2}}}{b_{k}}\left\langle\Psi_{f}, \psi_{k}(T)\right\rangle, \forall k \geqslant 4, \\
\int_{0}^{T} \dot{v}(t) \mathrm{e}^{i\left(\lambda_{k}-\lambda_{3}\right) t} \mathrm{~d} t & =\frac{i \sqrt{\theta_{3}}}{c_{k}}\left\langle\Psi_{f}, \psi_{k}(T)\right\rangle, \forall k \geqslant 4,
\end{aligned}\right.
$$

where $C$ is a complex number with

$$
\Re(C):=\frac{1}{2 b_{3} \sqrt{\theta_{2} \theta_{3}}} \Im\left(\left\langle\Psi_{f},\left(\sqrt{1-\theta_{2}-\theta_{3}} \psi_{1}-\sqrt{\theta_{2}} \psi_{2}-\sqrt{\theta_{3}} \psi_{3}\right)(T)\right\rangle\right) .
$$

This trigonometric moment problem has a solution $v \in \overline{H_{0}^{1}}((0, T), \mathbb{R})$, as soon as the right hand side belongs to $l^{2}$ and $T>2 /(3 \pi)$ (see [30], Th. 1.2.18), which is the case when $\Psi_{f} \in H_{(0)}^{3}((0,1), \mathbb{C})$. The assumption $T>2 /(3 \pi)$ corresponds to

$$
T>\frac{2 \pi}{D} \text { where } D:=\liminf _{j \rightarrow+\infty}\left(\omega_{j+1}-\omega_{j}\right)=\lambda_{2}-\lambda_{1}=3 \pi^{2}
$$

and $\left(\omega_{j}\right)_{j \in \mathbb{N}}$ is the increasing sequence of the frequencies in the trigonometric moment problem (2.6). 
Remark 4. The minimal time for the controllability of the linear system $\left(\Sigma_{\theta_{2}, \theta_{3}}\right)$, with $\left(\theta_{2}, \theta_{3}\right) \in \operatorname{Int}(\mathcal{D})$, may not be $2 /(3 \pi)$. We conjecture that $\left(\Sigma_{\theta_{2}, \theta_{3}}\right)$ is controllable in any positive time $T>0$. The proof of this conjecture could rely on an Ingham inequality of the form : let $T>0$, there exists $C>0$ such that, for every $N \in \mathbb{N}^{*}$ and for every $\left(\alpha_{j}\right)_{4 \leqslant|j| \leqslant N} \subset \mathbb{C}$,

$$
C \sum_{j=-N}^{N}\left|\alpha_{j}\right|^{2} \leqslant \frac{1}{2 T} \int_{-T}^{T}\left|\sum_{4 \leqslant|j| \leqslant N} \alpha_{j} z_{j}(t)\right|^{2} \mathrm{~d} t
$$

with

$$
\begin{aligned}
& z_{j}(t):=j^{3} a_{j} \sqrt{1-\theta_{2}-\theta_{3}} \mathrm{e}^{-i\left(\lambda_{j}-\lambda_{1}\right) t}+j^{3} b_{j} \sqrt{\theta_{2}} \mathrm{e}^{-i\left(\lambda_{j}-\lambda_{2}\right) t}+j^{3} c_{j} \sqrt{\theta_{3}} \mathrm{e}^{-i\left(\lambda_{j}-\lambda_{3}\right) t}, \forall j \geqslant 4, \\
& z_{j}(t):=\overline{z_{-j}(t)}, \forall j \leqslant-4 .
\end{aligned}
$$

The validity of such an inequality is an open problem.

Remark 5. At this step, we can justify the non controllability of the linearized system around $\sqrt{1-\theta} \psi_{1}+\sqrt{\theta} \psi_{2}$ stated in Remark 3. Let $\Psi$ be a solution of $\left(\Sigma_{\theta, 0}\right)$ with $0<\theta<1$, with some $v \in \overline{H_{0}^{1}}((0, T), \mathbb{R})$ and such that $\Psi(0)=0$. Let $\xi_{\theta}:=\sqrt{1-\theta} \psi_{1}-\sqrt{\theta} \psi_{2}$. Then

$$
\left\langle\Psi(T), \xi_{\theta}(T)\right\rangle=2 \sqrt{\theta(1-\theta)}\left\langle x^{2} \varphi_{1}, \varphi_{2}\right\rangle \int_{0}^{T} \dot{v}(t) \sin \left[\left(\lambda_{2}-\lambda_{1}\right) t\right] \mathrm{d} t \in \mathbb{R} .
$$

This condition is not implied by $\left.\Psi(T) \in T_{\mathcal{S}}\left(\sqrt{1-\theta} \psi_{1}+\sqrt{\theta} \psi_{2}\right)(T)\right)$.

Proposition 2. Let $T>0$ and $\left(\theta_{2}, \theta_{3}\right) \in \operatorname{Int}(\mathcal{D})$. The system $\left(\Sigma_{\theta_{2}, \theta_{3}}\right)$ (resp. $\left(\Sigma_{0,0}\right)$ ) is not controllable (resp. controllable up to codimension one) in $H_{(0)}^{3}((0,1), \mathbb{C})$ with control functions in $\overline{H_{0}^{1}} \cap H^{2}((0, T), \mathbb{R})$.

Proof. Let us assume that this is not the case for some $\left(\theta_{2}, \theta_{3}\right) \in \mathcal{D}$. Then, for every $\Psi_{f} \in H_{(0)}^{3}((0,1), \mathbb{R})$, there exists $v \in \overline{H_{0}^{1}} \cap H^{2}((0, T), \mathbb{R})$ which solves $(2.3)$ for every $k \geqslant 3$. However, an integration by parts shows that, for $w \in H^{1}((0, T), \mathbb{R})$,

$$
\left|\int_{0}^{T} w(t) \mathrm{e}^{i \lambda_{k} t} \mathrm{~d} t\right| \leqslant \frac{C}{k^{2}}\|w\|_{H^{1}((0, T), \mathbb{R})} .
$$

Thus, there exists a constant $C=C(\theta)>0$ such that, for every $\Psi_{f} \in H_{(0)}^{3}((0,1), \mathbb{R})$,

$$
\left|\left\langle\Psi_{f}, \varphi_{k}\right\rangle\right| \leqslant \frac{C}{k^{5}}
$$

We get a contradiction by considering, for instance, the function $\Psi_{f} \in H_{(0)}^{4}((0,1), \mathbb{C})$ with $\Psi_{f}^{(4)}=f$ and $f \in L^{2}((0,1), \mathbb{C})$ is defined by

$$
f:=\sum_{k \in Q} \frac{1}{\sqrt{k}} \varphi_{k}, \text { where } Q:=\left\{m^{2} ; m \in \mathbb{N}^{*}\right\}
$$

\section{The Nash-Moser theorem Used}

In order to get local controllability for the nonlinear system $(\Sigma)$ around $\sqrt{1-\theta_{2}-\theta_{3}} \psi_{1}+\sqrt{\theta_{2}} \psi_{2}+\sqrt{\theta_{3}} \psi_{3}$, we use a Nash-Moser theorem inspired from Hörmander's one in [26]. The introduction of a projection $\mathcal{P}$ in this statement introduces changes in the proof, so, we repeat it completely. Similar statements has already been used in [8-10]. We refer to [2] for another presentation and other applications of this theorem, the authors also explain how to detect the "Nash-Moser symptom". 
We consider a family of Hilbert spaces $\left(E_{a}\right)_{a \in[2,8]}$ with continuous injections $E_{b} \rightarrow E_{a}$ of norm $\leqslant 1$ when $b \geqslant a$. We suppose that we have linear operators $S_{\theta}: E_{2} \rightarrow E_{8}$ for $\theta \geqslant 1$. We also assume there exists a constant $K>0$ such that, for every $a, b \in[2,8]$ and for every $u \in E_{a}$,

$$
\begin{gathered}
\left\|S_{\theta} u\right\|_{b} \leqslant K\|u\|_{a} \text { when } b \leqslant a, \\
\left\|S_{\theta} u\right\|_{b} \leqslant K \theta^{b-a}\|u\|_{a} \text { when } b>a \\
\left\|u-S_{\theta} u\right\|_{b} \leqslant K \theta^{b-a}\|u\|_{a} \text { when } b<a, \\
\left\|\frac{\mathrm{d}}{\mathrm{d} \theta} S_{\theta} u\right\|_{b} \leqslant K \theta^{b-a-1}\|u\|_{a} .
\end{gathered}
$$

We fix a sequence $\left(\theta_{j}\right)_{j \in \mathbb{N}}$ of the form $\theta_{j}:=(j+1)^{\delta}$ where $0<\delta$ and we set, for every $j \in \mathbb{N}, \Delta_{j}:=\theta_{j+1}-\theta_{j}$. For every $u \in E_{a}$, we have a decomposition

$$
u=\sum_{j=0}^{\infty} \Delta_{j} R_{j} u
$$

with convergence in $E_{b}$ when $b<a$, moreover there exists a constant $K^{\prime}$ such that, for every $b \in[2,8]$,

$$
\left\|R_{j} u\right\|_{b} \leqslant K^{\prime} \theta_{j}^{b-a-1}\|u\|_{a}
$$

We also have the convexity of the norms: there exists a constant $c \geqslant 1$ such that, for every $a, b \in[2,8]$ with $a<b, \lambda \in[0,1]$, and $u \in E_{b}$,

$$
\|u\|_{\lambda a+(1-\lambda) b} \leqslant c\|u\|_{a}^{\lambda}\|u\|_{b}^{1-\lambda} .
$$

We refer to [26] for the proof of the two previous properties.

We have another family $\left(F_{a}\right)_{a \in[2,8]}$ with the same properties as above, we use the same notations for the smoothing operators. Moreover, we assume that the injection $F_{b} \rightarrow F_{a}$ is compact when $b>a$.

Theorem 7. Let $\mathcal{P}$ be a continuous linear operator from $F_{b}$ to $F_{b}$ of norm $\leqslant 1$ for $b=2, \ldots, 8$, such that $\mathcal{P} S_{\theta}=S_{\theta} \mathcal{P}$ for every $\theta$. Let $\beta$ be a real number such that

$$
5<\beta<6
$$

Let $V$ be a $E_{4}$-neighborhood of zero and $\Phi$ a map from $V$ to $F_{4}$ which is twice differentiable and satisfies

$$
\left\|\Phi^{\prime \prime}(u ; v, w)\right\|_{6} \leqslant C \sum\left(1+\|u\|_{m}\right)\|v\|_{m^{\prime}}\|w\|_{m^{\prime \prime}}
$$

where the sum is taken over the following values

\begin{tabular}{|c|c|c|}
\hline$m$ & $m^{\prime}$ & $m^{\prime \prime}$ \\
\hline 6 & 2 & 2 \\
\hline 4 & 4 & 4 \\
\hline 4 & 6 & 2 \\
\hline 4 & 2 & 6 \\
\hline
\end{tabular}

We assume that $\Phi: E_{4} \rightarrow F_{4}$ is continuous for every $a \in[2,8]$. We assume that, for every $v \in V \cap E_{8}, \Phi^{\prime}(v)$ has a right inverse $\psi(v): F_{7} \rightarrow E_{6}$, that $(v, g) \mapsto \psi(v, g)$ is continuous from $\left(V \cap E_{6}\right) \times F_{7} \rightarrow E_{6}$ and that there 
exists a constant $C$ such that, for every $(v, g) \in\left(V \cap E_{6}\right) \times F_{7}$,

$$
\begin{gathered}
\|\psi(v) g\|_{2} \leqslant C\left[\|\mathcal{P} g\|_{3}+\|v\|_{4}\|g\|_{3}\right] \\
\|\psi(v) g\|_{6} \leqslant C\left[\|\mathcal{P} g\|_{7}+\|v\|_{4}\|g\|_{7}+\|v\|_{8}\|g\|_{3}\right] .
\end{gathered}
$$

Then, there exists $\mathcal{C}>0$ and $\epsilon>0$ such that for every $f \in F_{\beta}$ with $\|f\|_{\beta}<\epsilon$, there exists $u \in E_{4}$ such that $\Phi(u)=\Phi(0)+f$ and $\|u\|_{4} \leqslant \mathcal{C}\|\mathcal{P} f\|_{\beta}$.

The inequalities (3.8) and (3.9) are called "tame estimates".

Proof. Let $g \in F_{\beta}$. There exists a decomposition

$$
g=\sum_{j=0}^{\infty} \Delta_{j} g_{j} \text { with }\left\|g_{j}\right\|_{b} \leqslant K^{\prime}\|g\|_{\beta} \theta_{j}^{b-\beta-1}, \forall b \in[2,8]
$$

Since $\mathcal{P} S_{\theta}=S_{\theta} \mathcal{P}$, we also have

$$
\mathcal{P} g=\sum_{j=0}^{\infty} \Delta_{j} \mathcal{P} g_{j} \text { with }\left\|\mathcal{P} g_{j}\right\|_{b} \leqslant K^{\prime}\|\mathcal{P} g\|_{\beta} \theta_{j}^{b-\beta-1}, \forall b \in[2,8]
$$

We claim that, when $\|g\|_{\beta}$ is small enough, we can define a sequence $\left(u_{j}\right)_{j \in \mathbb{N}}$ with $u_{0}=0$ and the recursive formula

$$
u_{j+1}:=u_{j}+\Delta_{j} \dot{u}_{j}, \quad \dot{u}_{j}:=\psi\left(v_{j}\right) g_{j}, \quad v_{j}:=S_{\theta_{j}} u_{j} .
$$

We also claim that there exist constants $C_{1}, C_{2}, C_{3}, C_{4}$ such that, for every $j \in \mathbb{N}^{*}$,

$$
\begin{gathered}
\left\|\dot{u}_{j}\right\|_{a} \leqslant C_{1}\|\mathcal{P} g\|_{\beta} \theta_{j}^{a-\beta}, \forall a \in\{2,4,6\}, \\
\left\|u_{j}\right\|_{4} \leqslant C_{2}\|\mathcal{P} g\|_{\beta} \text { and }\left\|u_{j}\right\|_{6} \leqslant C_{2}\|\mathcal{P} g\|_{\beta} \theta_{j}^{7-\beta}, \\
\left\|v_{j}\right\|_{4} \leqslant C_{3}\|\mathcal{P} g\|_{\beta},\left\|v_{j}\right\|_{a} \leqslant C_{3}\|\mathcal{P} g\|_{\beta} \theta_{j}^{a-\beta+1} \forall a \in\{6,8\}, \\
\left\|u_{j}-v_{j}\right\|_{a} \leqslant C_{4}\|\mathcal{P} g\|_{\beta} \theta_{j}^{a-\beta+1}, \forall a \in\{2,4,6\} .
\end{gathered}
$$

More precisely, we prove by induction on $k \in \mathbb{N}$ the following property

$$
\begin{aligned}
\mathcal{P}_{k}: & u_{j} \text { is well defined for } j=0, \ldots, k+1, \\
& (3.12) \text { is satisfied for } j=0, \ldots, k, \\
& (3.13),(3.14) \text { and }(3.15) \text { are satisfied for } j=0, \ldots, k+1 .
\end{aligned}
$$

We introduce $r>0$ such that, for every $u \in E_{4},\|u\|_{\alpha}<r$ implies $u \in V$.

Property $\mathcal{P}_{0}$ is obvious. Let $k \in \mathbb{N}^{*}$. We assume property $\mathcal{P}_{k-1}$ is satisfied. Let us prove $\mathcal{P}_{k}$.

The vector $u_{k+1}$ is well defined if and only if $v_{k} \in V$, which is true as soon as $\|\mathcal{P} g\|_{\beta}<r / C_{3}$ thanks to (3.14) with $j=k$.

Let us prove (3.12) for $j=k$. Using (3.8) and (3.10), we get

$$
\left\|\dot{u}_{k}\right\|_{2} \leqslant C K^{\prime}\|\mathcal{P} g\|_{\beta} \theta_{k}^{2-\beta}\left(1+C_{3}\|g\|_{\beta}\right) \leqslant 2 C K^{\prime}\|\mathcal{P} g\|_{\beta} \theta_{k}^{2-\beta},
$$

when $\|g\|_{\beta}<1 / C_{3}$. Using (3.9), (3.14), (3.10), we get

$$
\left\|\dot{u}_{k}\right\|_{6} \leqslant C K^{\prime}\|\mathcal{P} g\|_{\beta}\left[\theta_{k}^{6-\beta}+C_{3}\|g\|_{\beta} \theta_{k}^{6-\beta}+C_{3}\|g\|_{\beta} \theta_{k}^{9-\beta} \theta_{k}^{2-\beta}\right] \leqslant 3 C K^{\prime}\|\mathcal{P} g\|_{\beta} \theta_{k}^{6-\beta},
$$


when $\|g\|_{\beta}<1 / C_{3}$. Then, the convexity of the norm (3.5) provides

$$
\left\|\dot{u}_{k}\right\|_{4} \leqslant c 3 C K^{\prime}\|\mathcal{P} g\|_{\beta} \theta_{k}^{4-\beta} .
$$

Therefore, we have (3.12) for $j=k$, when $\|g\|_{\beta}<1 / C_{3}$ for $C_{1}=3 c C K^{\prime}$.

Let us prove (3.13) for $j=k+1$. Thanks to (3.12), we have

$$
\left\|u_{k+1}\right\|_{4} \leqslant C_{1}\|\mathcal{P} g\|_{\beta} \sum_{j=0}^{k} \Delta_{j} \theta_{j}^{4-\beta} \leqslant C_{1}\|\mathcal{P} g\|_{\beta} S,
$$

where $S:=\sum_{j=0}^{\infty} \Delta_{j} \theta_{j}^{4-\beta}$ is finite because $\beta>5$. Thanks to (3.12), we have

$$
\left\|u_{k+1}\right\|_{6} \leqslant C_{1}\|\mathcal{P} g\|_{\beta} \sum_{j=0}^{k} \Delta_{j} \theta_{j}^{6-\beta} \leqslant C_{1}\|\mathcal{P} g\|_{\beta} \frac{\theta_{k+1}^{7-\beta}}{7-\beta} .
$$

Thus, we get (3.13) for $j=k$ with

$$
C_{2}:=C_{1} \max \left\{S, \frac{1}{7-\beta}\right\} .
$$

We get (3.14) for $j=k+1$ thanks to (3.1) and (3.2), with $C_{3}:=K C_{2}$. We get (3.15) for $j=k+1$ thanks to (3.13) and (3.14) for $a=6$ and thanks to (3.3) and (3.13) for $a \in\{2,4\}$, with $C_{4}:=\max \left\{C_{2}+C_{3} ; K C_{2}\right\}$.

Inequality (3.12) proves that $\left(u_{k}\right)$ converges in $E_{4}$ toward

$$
u:=\sum_{j=0}^{\infty} \Delta_{j} \dot{u}_{j}, \text { which satisfies }\|u\|_{4} \leqslant C_{2}\|\mathcal{P} g\|_{\beta} .
$$

The continuity of the map $\Phi: E_{4} \rightarrow F_{4}$ implies that $\Phi\left(u_{k}\right)$ converges to $\Phi(u)$ in $F_{4}$.

Let us study the limit of the sequence $\left(\Phi\left(u_{k}\right)\right)_{k \in \mathbb{N}}$ in a different way. We have

$$
\Phi\left(u_{j+1}\right)-\Phi\left(u_{j}\right)=\Delta_{j}\left(e_{j}^{\prime}+e_{j}^{\prime \prime}+g_{j}\right)
$$

where

$$
\begin{gathered}
e_{j}^{\prime}:=\frac{1}{\Delta_{j}}\left(\Phi\left(u_{j}+\Delta_{j} \dot{u}_{j}\right)-\Phi\left(u_{j}\right)-\Phi^{\prime}\left(u_{j}\right) \Delta_{j} \dot{u}_{j}\right)=\Delta_{j} \int_{0}^{1}(1-t) \Phi^{\prime \prime}\left(u_{j}+t \Delta_{j} \dot{u}_{j} ; \dot{u}_{j}, \dot{u}_{j}\right) \mathrm{d} t \\
e_{j}^{\prime \prime}:=\left(\Phi^{\prime}\left(u_{j}\right)-\Phi^{\prime}\left(v_{j}\right)\right) \dot{u}_{j}=\int_{0}^{1} \phi^{\prime \prime}\left(v_{j}+t\left(u_{j}-v_{j}\right) ; u_{j}-v_{j}, \dot{u}_{j}\right) .
\end{gathered}
$$

Thanks to (3.6), we have

$$
\begin{aligned}
\left\|e_{j}^{\prime}\right\|_{6} & \leqslant C \sum\left(1+\left\|u_{j}\right\|_{m}+\Delta_{j}\left\|\dot{u}_{j}\right\|_{m}\right)\left\|\dot{u}_{j}\right\|_{m^{\prime}}\left\|\dot{u}_{j}\right\|_{m^{\prime \prime}} \\
& \leqslant C\left[\left(1+\left(C_{1}+C_{2}\right)\|\mathcal{P} g\|_{\beta} \theta_{j}^{7-\beta}\right) C_{1}^{2}\|\mathcal{P} g\|_{\beta}^{2} \theta_{j}^{4-2 \beta}+3\left(1+\left(C_{1}+C_{2}\right)\|\mathcal{P} g\|_{\beta}\right) C_{1}^{2}\|\mathcal{P} g\|_{\beta}^{2} \theta_{j}^{8-2 \beta}\right] \\
& \leqslant C\|\mathcal{P} g\|_{\beta}^{2} \theta_{j}^{8-2 \beta} \\
\left\|e_{j}^{\prime \prime}\right\|_{6} \leqslant & C \sum\left(1+\left\|v_{j}\right\|_{m}+\left\|u_{j}-v_{j}\right\|_{m}\right)\left\|u_{j}-v_{j}\right\|_{m^{\prime}}\left\|\dot{u}_{j}\right\|_{m^{\prime \prime}} \\
\leqslant & C\left[\left(1+\left(C_{3}+C_{4}\right)\|\mathcal{P} g\|_{\beta} \theta_{j}^{7-\beta}\right) C_{1} C_{4}\|\mathcal{P} g\|_{\beta}^{2} \theta_{j}^{5-2 \beta}+3\left(1+\left(C_{3}+C_{4}\right)\|\mathcal{P} g\|_{\beta}\right) C_{1} C_{4}\|\mathcal{P} g\|_{\beta}^{2} \theta_{j}^{9-2 \beta}\right] \\
\leqslant & C\|\mathcal{P} g\|_{\beta}^{2} \theta_{j}^{9-2 \beta} .
\end{aligned}
$$


Since $9-2 \beta<-1$, then $\sum \Delta_{j}\left(e_{j}^{\prime}+e_{j}^{\prime \prime}\right)$ converges in $F_{6}$ and

$$
\left\|\sum_{j=0}^{\infty} \Delta_{j}\left(e_{j}^{\prime}+e_{j}^{\prime \prime}\right)\right\|_{6} \leqslant C\|\mathcal{P} g\|_{\beta}^{2} .
$$

The uniqueness of the limit of the sequence $\left(\Phi\left(u_{k}\right)\right)_{k \in \mathbb{N}}$ gives the following equality in $F_{4}$

$$
\Phi(u)=g+T(g)
$$

where $T(g) \in F_{6}$ and

$$
\|T(g)\|_{6} \leqslant C\|\mathcal{P} g\|_{\beta}^{2} .
$$

Let $0<\rho<\min \left\{1 /(2 C), r / C_{3}, 1 / C_{3}\right\}$ where $C$ is given by (3.16). Let $f \in F_{\beta}$, be such that $\|f\|_{\beta}<\rho / 2$. Then the map $\Theta(g):=f-T(g)$ maps the ball $B:=\left\{g \in F_{\beta} ;\|g\|_{\beta} \leqslant \rho\right\}$ into itself. The Leray-Schauder fixed point theorem justifies that $\Theta$ has a fix point $g \in B$. The equality $g=f+T(g)$ and the choice of $\rho$ gives $\|\mathcal{P} g\|_{\beta} \leqslant 2\|\mathcal{P} f\|_{\beta}$. The vector $u$ built in the first part of this proof gives the solution and satisfies $\|u\|_{4} \leqslant 2 C_{2}\|\mathcal{P} f\|_{\beta}$.

Remark 6. The proof can be done thanks to the Banach fixed point theorem, instead of the Leray-Schauder fixed point theorem, provided one add new assumptions (see next proposition). In this situation, one does not need any longer the compactness of the injections $F_{b} \rightarrow F_{a}$ for $b>a$. The interest of this approach is that it provides the continuity of the local inverse $f \mapsto u$ of the map $\Phi$. This continuity is important for the use of the intermediate values theorem in Section 5.3.

Theorem 8. Let us consider the same assumptions as in the previous theorem. We assume moreover that, for every $u, \tilde{u} \in V \cap E_{6}$,

$$
\left\|\Phi^{\prime \prime}(u ; v, w)-\Phi^{\prime \prime}(\tilde{u} ; v, w)\right\|_{6} \leqslant C \sum\left(1+\|u-\tilde{u}\|_{m}\right)\|v\|_{m^{\prime}}\|w\|_{m^{\prime \prime}}
$$

where the sum is taken over the values given in (3.7). We also assume that, for every $v, \tilde{v} \in V \cap E_{8}$,

$$
\begin{gathered}
\|[\psi(v)-\psi(\tilde{v})] g\|_{2} \leqslant C\|v-\tilde{v}\|_{4}\|g\|_{3}, \\
\|[\psi(v)-\psi(\tilde{v})] g\|_{6} \leqslant C\left[\|v-\tilde{v}\|_{4}\|g\|_{7}+\|v-\tilde{v}\|_{8}\|g\|_{3}\right] .
\end{gathered}
$$

Then, there exists $\mathcal{C}>0, \eta>0$ and a continuous map

$$
\begin{aligned}
\Pi: \mathcal{V} & \rightarrow E_{4} \text { where } \mathcal{V}:=\left\{f \in F_{\beta} ;\|f\|_{\beta}<\eta\right\} \\
f & \mapsto u
\end{aligned}
$$

such that, for every $f \in \mathcal{V}$,

$$
\Phi(\Pi(f))=\Phi(0)+f \quad \text { and } \quad\|\Pi(f)\|_{4} \leqslant \mathcal{C}\|\mathcal{P} f\|_{\beta} .
$$

Proof. The map $\Pi$ is the composition of the two following maps

$$
\begin{aligned}
& F_{\beta} \rightarrow F_{\beta} \rightarrow E_{4} \\
& f \mapsto g \mapsto u
\end{aligned}
$$

where $f=g+T(g)$ and $u$ is the limit built in the previous proof. First, we prove the continuity of the second map $g \mapsto u$. Let $g, \tilde{g} \in F_{\beta}$ and $\left(u_{j}\right),\left(\dot{u}_{j}\right),\left(v_{j}\right),\left(\tilde{u}_{j}\right),\left(\tilde{u}_{j}\right),\left(\tilde{v}_{j}\right)$ be the sequences built in the proof of Theorem 7 . 
In the same way as we proved (3.12), (3.13), (3.14) (3.15) thanks to (3.8) and (3.9), we prove the existence of $C_{1}, C_{2}, C_{3}, C_{4}>0$ such that, for every $j \in \mathbb{N}$,

$$
\begin{gathered}
\left\|\dot{u}_{j}-\tilde{u}_{j}\right\|_{a} \leqslant C_{1}\|g-\tilde{g}\|_{\beta} \theta_{j}^{a-\beta}, \forall a \in\{2,4,6\}, \\
\left\|u_{j}-\tilde{u}_{j}\right\|_{4} \leqslant C_{2}\|g-\tilde{g}\|_{\beta} \text { and }\left\|u_{j}-\tilde{u}_{j}\right\|_{6} \leqslant C_{2}\|g-\tilde{g}\|_{\beta} \theta_{j}^{7-\beta}, \\
\left\|v_{j}-\tilde{v}_{j}\right\|_{4} \leqslant C_{3}\|g-\tilde{g}\|_{\beta},\left\|v_{j}-\tilde{v}_{j}\right\|_{a} \leqslant C_{3}\|g-\tilde{g}\|_{\beta} \theta_{j}^{a-\beta+1} \forall a \in\{6,8\}, \\
\left\|\left(u_{j}-v_{j}\right)-\left(\tilde{u}_{j}-\tilde{v}_{j}\right)\right\|_{a} \leqslant C_{4}\|g-\tilde{g}\|_{\beta} \theta_{j}^{a-\beta+1}, \forall a \in\{2,4,6\} .
\end{gathered}
$$

In particular, we get

$$
\|u-\tilde{u}\|_{4} \leqslant C_{2}\|g-\tilde{g}\|_{\beta}
$$

which gives the continuity of the second map of (3.21). Now, we prove the continuity of the first map $f \mapsto g$ of (3.21). It is sufficient to prove that the map $T: F_{\beta} \rightarrow F_{\beta}$ is a contraction, indeed, the inequality

$$
\|T(g)-T(\tilde{g})\|_{\beta} \leqslant \delta\|g-\tilde{g}\|_{\beta}
$$

with $\delta \in(0,1)$ gives

$$
\|g-\tilde{g}\|_{\beta} \leqslant \frac{1}{1-\delta}\|f-\tilde{f}\|_{\beta} .
$$

We have

$$
T(g)-T(\tilde{g})=\sum_{j=0}^{\infty} \Delta_{j}\left[\left(e_{j}^{\prime}-\tilde{e}_{j}^{\prime}\right)+\left(e_{j}^{\prime \prime}-\tilde{e}_{j}^{\prime \prime}\right)\right] .
$$

Let us prove the existence of $C_{5}, C_{6}>0$ such that, for every $j \in \mathbb{N}$,

$$
\begin{aligned}
& \left\|e_{j}^{\prime}-\tilde{e}_{j}^{\prime}\right\|_{6} \leqslant C_{5} \max \left\{\|g\|_{\beta},\|\tilde{g}\|_{\beta}\right\}\|g-\tilde{g}\|_{\beta}, \\
& \left\|e_{j}^{\prime \prime}-\tilde{e}_{j}^{\prime \prime}\right\|_{6} \leqslant C_{6} \max \left\{\|g\|_{\beta},\|\tilde{g}\|_{\beta}\right\}\|g-\tilde{g}\|_{\beta},
\end{aligned}
$$

which shows that $T$ is a contraction of a small neighborhood of zero in $F_{\beta}$. In order to prove the first bound of (3.26), we use (3.17) and the decomposition

$$
\begin{aligned}
e_{j}-\tilde{e}_{j}= & \Delta_{j} \int_{0}^{1}(1-t)\left[\Phi^{\prime \prime}\left(u_{j}+t \Delta_{j} \dot{u}_{j} ; \dot{u}_{j}, \dot{u}_{j}\right)-\Phi^{\prime \prime}\left(\tilde{u}_{j}+t \Delta_{j} \tilde{\dot{u}}_{j} ; \dot{u}_{j}, \dot{u}_{j}\right)\right] \mathrm{d} t+ \\
& \Delta_{j} \int_{0}^{1}(1-t) \Phi^{\prime \prime}\left(\tilde{u}_{j}+t \Delta_{j} \tilde{\dot{u}}_{j} ; \dot{u}_{j}-\tilde{\dot{u}}_{j}, \dot{u}_{j}\right) \mathrm{d} t+ \\
& \Delta_{j} \int_{0}^{1}(1-t) \Phi^{\prime \prime}\left(\tilde{u}_{j}+t \Delta_{j} \tilde{\dot{u}}_{j} ; \dot{u}_{j}-\tilde{\dot{u}}_{j}, \dot{u}_{j}-\tilde{\dot{u}}_{j}\right) \mathrm{d} t .
\end{aligned}
$$

The second bound of (3.26) can be proved in the same way. We know that

$$
\|\Pi(f)\|_{4} \leqslant C_{2}\|\mathcal{P} g\|_{\beta} .
$$

Thanks to (3.16), we have

$$
\|\mathcal{P} g\|_{\beta} \leqslant\|\mathcal{P} f\|_{\beta}+\|\mathcal{P} T g\|_{\beta} \leqslant\|\mathcal{P} f\|_{\beta}+C\|\mathcal{P} g\|_{\beta}^{2}
$$

which gives (3.20). 


\section{LocAl CONTROLLABILITy AROUND $\sqrt{1-\theta_{2}-\theta_{3}} \psi_{1}+\sqrt{\theta_{2}} \psi_{2}+\sqrt{\theta_{3}} \psi_{3}$ WITH $\left(\theta_{2}, \theta_{3}\right) \in \operatorname{INT}(\mathcal{D})$}

The aim of this section is the proof of Theorem 5 by applying Theorem 7 . In all this section, $\left(\theta_{2}, \theta_{3}\right) \in \operatorname{Int}(\mathcal{D})$ is fixed and we use the notations $\left(\Sigma_{r e f}\right)$ for $\left(\Sigma_{\theta_{2}, \theta_{3}}\right)$ and

$$
\psi_{\text {ref }}:=\sqrt{1-\theta_{2}-\theta_{3}} \psi_{1}+\sqrt{\theta_{2}} \psi_{2}+\sqrt{\theta_{3}} \psi_{3}
$$

\subsection{Context for the Nash-Moser theorem}

We apply Theorem 7 to the map $\Phi$ defined in Section 1.5.2, with $T:=2 / \pi$, in a neighborhood of $\psi_{\text {ref }}(0)$, with $\mathcal{P}=$ Id and with the spaces

$$
\begin{gathered}
E_{a}:=\left[\mathcal{S} \cap H_{(0)}^{a}((0,1), \mathbb{C})\right] \times \overline{H_{0}^{\frac{a}{2}}}((0, T), \mathbb{R}), \forall a \in\{2,4,6,8\}, \\
F_{a}:=\left[\mathcal{S} \cap H_{(0)}^{a}((0,1), \mathbb{C})\right] \times\left[\mathcal{S} \cap H_{(0)}^{a}((0,1), \mathbb{R})\right], \forall a \in\{2,3,4,5,6,7\},
\end{gathered}
$$

where $T:=2 / \pi$. We work on the manifold $\mathcal{S}$ instead of a whole space. It does not matter because, as in [9] and $[10]$, we can move the system to an hyperplane of $L^{2}((0,1), \mathbb{R})$ by studying

$$
\widetilde{\Phi}:=q \circ \Phi \circ r
$$

where $r\left(\psi_{0}, u\right)=\left(p^{-1}\left(\psi_{0}\right), u\right), q\left(\psi_{0}, \psi_{f}\right)=\left(p\left(\psi_{0}\right), p\left(\psi_{f}\right)\right)$, and $p$ is a suitable local diffeomorphism from a neighborhood of $\left\{\psi_{\text {ref }}(t) ; t \in[0,2 / \pi)\right\}$, in the sphere $\mathcal{S}$ to an hyperplane $\mathcal{H}$ of $L^{2}((0,1), \mathbb{C})$, which does not change too much the $H^{s}((0,1), \mathbb{C})$-norm. For example, one can use the following one.

Proposition 3. Let $\left(\theta_{2}, \theta_{3}\right) \in \operatorname{Int}(\mathcal{D}), \epsilon>0$ be small enough so that

$$
\begin{gathered}
\left(\sqrt{1-\theta_{2}-\theta_{3}}-\epsilon\right)^{2}(1-\epsilon)-\epsilon(1+\epsilon)^{2}>0, \\
(1-\epsilon)\left[\left(1-\theta_{2}-\theta_{3}\right)^{2}-\epsilon^{2}-\epsilon\right]>0, \\
\mathcal{U}:=\left\{\psi \in \mathcal{S} ; \exists t \in[0,2 / \pi),\left\|\psi-\psi_{r e f}(t)\right\|_{L^{2}((0,1), \mathbb{C})}<\epsilon\right\}, \\
\mathcal{H}:=\left\{\psi \in L^{2}((0,1), \mathbb{C}) ; \Re<\psi, \varphi_{4}>=0\right\}
\end{gathered}
$$

and $p: L^{2}((0,1), \mathbb{C}) \rightarrow \mathcal{H}$ be defined by

$$
p(\psi):=\psi-\Re\left(\left\langle\psi, \varphi_{4}\right\rangle\right) \varphi_{4}+\Re\left(\left\langle\psi, \varphi_{4}\right\rangle\right)\left\langle\psi, \varphi_{1}\right\rangle \varphi_{1} .
$$

The map $p$ is a $C^{1}$ diffeomorphism from $\mathcal{U}$ to an open subset of $\mathcal{H}$. Moreover, the norm of dp $(\psi)$ as a linear operator from $\left(T_{\mathcal{S}},\|\cdot\|_{H^{s}((0,1), \mathbb{C})}\right)$ to $\left(\mathcal{H},\|\cdot\|_{H^{s}((0,1), \mathbb{C})}\right)$ is uniformly bounded on $\mathcal{U}$, for every integer $s \in[2,9]$.

Proof. Let us introduce the orthogonal projection $P: L^{2}((0,1), \mathbb{C}) \rightarrow\left(\mathbb{C} \varphi_{1}+\mathbb{R} \varphi_{4}\right)^{\perp}$. First, we prove that $p$ is injective on $\mathcal{U}$. Let $\psi, \tilde{\psi} \in \mathcal{U}$ be such that $p(\psi)=p(\tilde{\psi})$. Then $P(\psi)=P(\tilde{\psi})$ and

$$
\left(1+\Re\left\langle\psi, \varphi_{4}\right\rangle\right)\left\langle\psi, \varphi_{1}\right\rangle=\left(1+\Re\left\langle\tilde{\psi}, \varphi_{4}\right\rangle\right)\left\langle\tilde{\psi}, \varphi_{1}\right\rangle
$$

The equality $\|\psi\|_{L^{2}}=\|\tilde{\psi}\|_{L^{2}}$ gives

$$
\left(\Re\left\langle\psi, \varphi_{4}\right\rangle\right)^{2}+\left|\left\langle\psi, \varphi_{1}\right\rangle\right|^{2}=\left(\Re\left\langle\tilde{\psi}, \varphi_{4}\right\rangle\right)^{2}+\left|\left\langle\tilde{\psi}, \varphi_{1}\right\rangle\right|^{2} .
$$


The relations (4.3) and (4.4) lead to

$$
\left|\left\langle\psi, \varphi_{1}\right\rangle\right|^{2}\left(\left(1+\Re\left\langle\tilde{\psi}, \varphi_{4}\right\rangle\right)^{2}-\left(1+\Re\left\langle\psi, \varphi_{4}\right\rangle\right)^{2}\right)=\left(1+\Re\left\langle\tilde{\psi}, \varphi_{4}\right\rangle\right)^{2}\left[\left(\Re\left\langle\tilde{\psi}, \varphi_{4}\right\rangle\right)^{2}-\left(\Re\left\langle\psi, \varphi_{4}\right\rangle\right)^{2} .\right.
$$

We assume that $\psi \neq \tilde{\psi}$. Then $\Re\left\langle\tilde{\psi}, \varphi_{4}\right\rangle \neq \Re\left\langle\psi, \varphi_{4}\right\rangle$, otherwise (4.3) gives $\left\langle\tilde{\psi}, \varphi_{1}\right\rangle=\left\langle\psi, \varphi_{1}\right\rangle$ thus $\tilde{\psi}=\psi$. Therefore, $y:=\Re\left\langle\tilde{\psi}, \varphi_{4}\right\rangle$ is a solution in $[-\epsilon, \epsilon]$ of $f(y)=0$ where

$$
f(y):=(1+y)^{2}(b+y)-a(2+b+y), \quad a:=\left|\left\langle\psi, \varphi_{1}\right\rangle\right|, \quad b:=\Re\left\langle\psi, \varphi_{4}\right\rangle .
$$

The assumption (4.1) justifies that $f(y)<0$ for every $y \in[-\epsilon, \epsilon]$, which is a contradiction.

Now, we prove that, for every $\psi \in \mathcal{U}, d p(\psi)$ is an isomorphism from $T_{\mathcal{S}}(\psi)$ to $\mathcal{H}$. Let $\psi \in \mathcal{U}$ and $\xi \in \mathcal{H}$. For $h \in L^{2}((0,1), \mathbb{C})$, the statement $\left(h \in T_{\mathcal{S}} \psi\right.$ and $\left.d p(\psi) h=\xi\right)$ is equivalent to $P h=P \xi$ and $A X=b$ where

$$
\begin{gathered}
A:=\left(\begin{array}{ccc}
\Re\left\langle\psi, \varphi_{1}\right\rangle & \Im\left\langle\psi, \varphi_{1}\right\rangle & \Re\left\langle\psi, \varphi_{4}\right\rangle \\
1+\Re\left\langle\psi, \varphi_{4}\right\rangle & 0 & \Re\left\langle\psi, \varphi_{1}\right\rangle \\
0 & 1+\Re\left\langle\psi, \varphi_{4}\right\rangle & \Im\left\langle\psi, \varphi_{1}\right\rangle
\end{array}\right) \\
X:=\left(\begin{array}{c}
\Re\left\langle h, \varphi_{1}\right\rangle \\
\Im\left\langle h, \varphi_{1}\right\rangle \\
\Re\left\langle h, \varphi_{4}\right\rangle
\end{array}\right), \quad b:=\left(\begin{array}{c}
-\Re\langle P \xi, P \psi\rangle \\
\Re\left\langle\xi, \varphi_{1}\right\rangle \\
\Im\left\langle\xi, \varphi_{1}\right\rangle
\end{array}\right) .
\end{gathered}
$$

Thanks to $(4.2)$, we have $\operatorname{det}(A)<0$. We conclude thanks to the inverse mapping theorem.

It is clear that $\|d p(\psi)\|_{H^{s} \rightarrow H^{s}} \leqslant 1+\left\|\varphi_{1}\right\|_{H^{s}}+\left\|\varphi_{4}\right\|_{H^{s}}$. Since $\|P \xi\|_{H^{s}} \leqslant\|\xi\|_{H^{s}}$ and $\left\|A^{-1}\right\|$ is uniformly bounded with respect to $\psi \in \mathcal{U}$, then $\left\|d p(\psi)^{-1}\right\|_{H^{s} \rightarrow H^{s}}$ also.

For the construction of smoothing operators for the controls $u \in \overline{H_{0}^{1}}((0, T), \mathbb{R})$, we can use the same strategy as in [9], Section 3.3, which is inspired from [24]. For smoothing operators on the wave functions, we propose

$$
S_{\theta} \varphi:=\sum_{k=1}^{\infty} s\left(\frac{k}{\theta}\right)\left\langle\varphi, \varphi_{k}\right\rangle \varphi_{k}
$$

where $s \in C^{\infty}\left(\mathbb{R}_{+},[0,1]\right), s \equiv 1$ on $[0,1]$ and $s \equiv 0$ on $[2,+\infty]$. The proof of $(3.1),(3.2),(3.3),(3.4)$ is the same as in [9], Section 3.3. Note that $S_{\theta}$ preserves the hyperplane $\mathcal{H}$ of Proposition 3.

\subsection{Bound on $\Phi^{\prime \prime}$}

The aim of this section is the proof of the bound (3.6) on the map $\Phi$ defined in Section 1.5.2.

Proposition 4. The map $\Phi: E_{6} \rightarrow F_{6}$ is twice differentiable and for every $\left(\psi_{0}, u\right) \in E_{6},\left(\phi_{0}, \nu\right),\left(\xi_{0}, \mu\right) \in E_{6}$, we have

$$
\Phi^{\prime \prime}\left(\psi_{0}, u\right) \cdot\left(\left(\phi_{0}, \nu\right),\left(\xi_{0}, \mu\right)\right)=(0, h(T))
$$

where

$$
\left\{\begin{array}{l}
i \dot{\psi}=-\psi^{\prime \prime}+\left(\dot{u}-4 u^{2}\right) x^{2} \psi \\
\psi(t, 0)=\psi(t, 1)=0 \\
\psi(0)=\psi_{0}
\end{array}\right.
$$




$$
\begin{aligned}
& \left\{\begin{array} { l } 
{ i \dot { \xi } = - \xi ^ { \prime \prime } + ( \dot { u } - 4 u ^ { 2 } ) x ^ { 2 } \xi + ( \dot { \mu } - 8 u \mu ) x ^ { 2 } \psi , } \\
{ \xi ( t , 0 ) = \xi ( t , 1 ) = 0 , } \\
{ \xi ( 0 ) = \xi _ { 0 } , }
\end{array} \quad \left\{\begin{array}{l}
i \dot{\phi}=-\phi^{\prime \prime}+\left(\dot{u}-4 u^{2}\right) x^{2} \phi+(\dot{\nu}-8 u \nu) x^{2} \psi, \\
\phi(t, 0)=\phi(t, 1)=0, \\
\phi(0)=\phi_{0},
\end{array}\right.\right. \\
& \left\{\begin{array}{l}
i \dot{h}=-h^{\prime \prime}+\left(\dot{u}-4 u^{2}\right) x^{2} h+(\dot{\mu}-8 u \mu) x^{2} \phi+(\dot{\nu}-8 u \nu) x^{2} \xi-4 \nu \mu x^{2} \psi, \\
\xi(t, 0)=\xi(t, 1)=0, \\
\xi(0)=\xi_{0} .
\end{array}\right.
\end{aligned}
$$

For every $r>0$ there exists a constant $C(r)>0$ such that, for every $\left(\psi_{0}, u\right) \in E_{6},\left(\phi_{0}, \nu\right),\left(\xi_{0}, \mu\right) \in E_{6}$ with $\left\|\left(\psi_{0}, u\right)\right\|_{4}<r$, we have

$$
\left\|\Phi^{\prime \prime}\left(\psi_{0}, u\right) .\left(\left(\phi_{0}, \nu\right),\left(\xi_{0}, \mu\right)\right)\right\|_{6} \leqslant C(r) \sum\left(1+\left\|\left(\psi_{0}, u\right)\right\|_{m}\right)\left\|\left(\phi_{0}, \nu\right)\right\|_{m^{\prime}}\left\|\left(\xi_{0}, \mu\right)\right\|_{m^{\prime \prime}}
$$

where the sum is taken over the values given in (3.7).

Proof. We only justify the bound (4.5). Thanks to Proposition 21, we have

$$
\|h(T)\|_{H_{(0)}^{6}} \leqslant C\left[\|f\|_{W^{2,1}\left((0, T), H_{(0)}^{2}\right)}+\|f\|_{C^{0}\left([0, T], H^{4}\right)}+\|u\|_{H^{3}}\|f\|_{L^{1}\left((0, T), H_{(0)}^{2}\right)}\right] .
$$

where $f:=f_{1}+f_{2}+f_{3}, f_{1}:=(\dot{\mu}-8 u \mu) x^{2} \phi, f_{2}:=(\dot{\nu}-8 u \nu) x^{2} \xi$ and $f_{3}:=-4 \nu \mu x^{2} \psi$. Using Propositions 19-21, we get

$$
\begin{aligned}
& \left\|f_{1}\right\|_{L^{1}\left((0, T), H^{2}\right)} \leqslant C\|\mu\|_{H^{1}} A_{2}, \\
& \left\|f_{1}\right\|_{C^{0}\left((0, T), H^{4}\right)} \leqslant C\|\mu\|_{H^{2}} A_{4}, \\
& \left\|f_{1}\right\|_{W^{2,1}\left((0, T), H^{2}\right)} \leqslant C\left[\|\mu\|_{H^{3}} A_{2}+\|\mu\|_{H^{2}} A_{4}+\|\mu\|_{H^{1}} A_{6}\right]
\end{aligned}
$$

with

$$
\begin{aligned}
A_{2} & :=\left\|\phi_{0}\right\|_{H^{2}}+\|\nu\|_{H^{1}} a_{2} \\
A_{4} & :=\left\|\phi_{0}\right\|_{H^{4}}+\|\nu\|_{H^{2}} a_{2}+\|\nu\|_{H^{1}} a_{4} \\
A_{6} & :=\left\|\phi_{0}\right\|_{H^{6}}+\|u\|_{H^{3}}\left\|\phi_{0}\right\|_{H^{2}}+\|\nu\|_{H^{3}} a_{2}+\|\nu\|_{H^{2}} a_{4}+\|\nu\|_{H^{1}} a_{6}
\end{aligned}
$$

and $a_{j}:=\left\|\left(\psi_{0}, u\right)\right\|_{E_{j}}$ for $j=2,4,6$. We have

$$
\begin{aligned}
& \left\|f_{3}\right\|_{L^{1}\left((0, T), H^{2}\right)} \leqslant C\|\mu \nu\|_{L^{1}} a_{2} \leqslant C\|\mu\|_{H^{1}}\|\nu\|_{H^{1}} a_{2}, \\
& \left\|f_{3}\right\|_{C^{0}\left((0, T), H^{4}\right)} \leqslant C\|\mu \nu\|_{C^{0}} a_{4} \leqslant C\|\mu\|_{H^{1}}\|\nu\|_{H^{1}} a_{4},
\end{aligned}
$$

$$
\begin{aligned}
\left\|f_{3}\right\|_{W^{2,1}\left((0, T), H^{2}\right)} & \leqslant C\left[\|\mu \nu\|_{H^{2}} a_{2}+\|\mu \nu\|_{H^{1}} a_{4}+\|\mu \nu\|_{L^{2}} a_{6}\right] \\
& \leqslant C\left[\left(\|\mu\|_{H^{2}}\|\nu\|_{H^{1}}+\|\mu\|_{H^{1}}\|\nu\|_{H^{2}}\right) a_{2}+\|\mu\|_{H^{1}}\|\nu\|_{H^{1}} a_{6}\right] .
\end{aligned}
$$

\subsection{Controllability of the linearized system around $\left(\psi_{r e f}, u \equiv 0\right)$ with tame estimates}

The goal of this section is the proof of the following proposition, which corresponds to the bounds (3.8) and (3.9) for $v=0$. We introduce, for $s>0$ the spaces

$$
h^{s}\left(\mathbb{N}^{*}, \mathbb{C}\right):=\left\{d=\left(d_{k}\right)_{k \in \mathbb{N}^{*}} \in l^{2}\left(\mathbb{N}^{*}, \mathbb{C}\right) ;\left(k^{s} d_{k}\right)_{k \in \mathbb{N}^{*}} \in l^{2}\left(\mathbb{N}^{*}, \mathbb{C}\right)\right\}
$$

equipped with the norm $\|d\|_{h^{s}\left(\mathbb{N}^{*}, \mathbb{C}\right)}:=\left\|k^{s} d_{k}\right\|_{l^{2}\left(\mathbb{N}^{*}, \mathbb{C}\right)}$. 
Proposition 5. Let $T:=2 / \pi$. There exists a constant $C>0$ such that, for every $\Psi_{f} \in H_{(0)}^{7}((0,1), \mathbb{C}) \cap$ $T_{\mathcal{S}}\left(\psi_{\text {ref }}(T)\right)$, there exists a trajectory $(\Psi, v)$ of $\left(\Sigma_{\text {ref }}\right)$ with $v \in \overline{H_{0}^{3}}((0, T), \mathbb{R}), \Psi(0)=0$ and $\Psi(T)=\Psi_{f}$, moreover

$$
\|v\|_{H_{0}^{1}((0, T), \mathbb{R})} \leqslant C\left\|\Psi_{f}\right\|_{H_{(0)}^{3}((0,1), \mathbb{C})} \quad \text { and } \quad\|v\|_{H_{0}^{3}((0, T), \mathbb{R})} \leqslant C\left\|\Psi_{f}\right\|_{H_{(0)}^{7}((0,1), \mathbb{C})} .
$$

Proof. Thanks to Section 2, it is sufficient to prove the existence of a constant $C>0$ such that, for every $d=\left(d_{k}\right)_{k \in \mathbb{N}} \in h^{4}(\mathbb{N}, \mathbb{C})$ there exists $\dot{v} \in H_{0}^{2}((0, T), \mathbb{R})$ with the following prescribed Fourier coefficients

$$
\left\{\begin{array}{l}
\int_{0}^{T} \dot{v}(t) \mathrm{d} t=0, \\
\int_{0}^{T} t \dot{v}(t) \mathrm{d} t=0, \\
\int_{0}^{T} \dot{v}(t) \mathrm{e}^{i\left(\lambda_{2}-\lambda_{1}\right) t} \mathrm{~d} t=d_{0} \\
\int_{0}^{T} \dot{v}(t) \mathrm{e}^{i\left(\lambda_{3}-\lambda_{1}\right) t} \mathrm{~d} t=d_{1} \\
\int_{0}^{T} \dot{v}(t) \mathrm{e}^{i\left(\lambda_{3}-\lambda_{2}\right) t} \mathrm{~d} t=d_{2} \\
\int_{0}^{T} \dot{v}(t) \mathrm{e}^{i\left(\lambda_{k}-\lambda_{1}\right) t} \mathrm{~d} t=d_{3(k-2)}, \forall k \geqslant 3, \\
\int_{0}^{T} \dot{v}(t) \mathrm{e}^{i\left(\lambda_{k}-\lambda_{2}\right) t} \mathrm{~d} t=d_{3(k-2)+1}, \forall k \geqslant 3, \\
\int_{0}^{T} \dot{v}(t) \mathrm{e}^{i\left(\lambda_{k}-\lambda_{3}\right) t} \mathrm{~d} t=d_{3(k-2)+2}, \forall k \geqslant 3,
\end{array}\right.
$$

and which satisfies

$$
\|\dot{v}\|_{L^{2}((0, T), \mathbb{R})} \leqslant C\|d\|_{l^{2}(\mathbb{N}, \mathbb{C})} \quad \text { and } \quad\|\dot{v}\|_{H_{0}^{2}((0, T), \mathbb{R})} \leqslant C\|d\|_{h^{4}\left(\mathbb{N}^{*}, \mathbb{C}\right)} .
$$

A candidate is

$$
\begin{aligned}
\dot{v}(t):= & \left\{d_{0} \mathrm{e}^{-i\left(\lambda_{2}-\lambda_{1}\right) t}+d_{1} \mathrm{e}^{-i\left(\lambda_{3}-\lambda_{1}\right) t}+d_{2} \mathrm{e}^{-i\left(\lambda_{3}-\lambda_{2}\right) t}+\sum_{k=3}^{\infty}\left[d_{3(k-2)} \mathrm{e}^{-i\left(\lambda_{k}-\lambda_{1}\right) t}\right.\right. \\
& \left.\left.+d_{3(k-2)+1} \mathrm{e}^{-i\left(\lambda_{k}-\lambda_{2}\right) t}+d_{3(k-2)+2} \mathrm{e}^{-i\left(\lambda_{k}-\lambda_{3}\right) t}\right]+ \text { c.c. }\right\}\left(1-\cos \left(\pi^{2} t\right)\right)
\end{aligned}
$$

where "+ c.c." means that we sum the complex conjugate number of the expression before.

Remark 7. In the same way as in Remark 4, the previous proposition probably holds for any $T>0$.

\subsection{Controllability of the linearized system around $(\psi, u)$, close to $\left(\psi_{r e f}, 0\right)$ in $E_{4}$, with tame estimates}

The aim of this section is the proof of the existence of a right inverse to the differential map $d \Phi\left(\psi_{0}, u\right)$ when $\left(\psi_{0}, u\right)$ is close enough to $\left(\psi_{r e f}(0), 0\right)$ in $E_{4}$, which satisfies (3.8) and (3.9).

Let $\left(\psi_{0}, u\right) \in E_{8}$, and $\psi$ be the solution of the Cauchy problem

$$
\left\{\begin{array}{l}
i \dot{\psi}=-\psi^{\prime \prime}+\left(\dot{u}-4 u^{2}\right)(t) x^{2} \psi, x \in(0,1), t \in(0, T) \\
\psi(t, 0)=\psi(t, 1)=0 \\
\psi(0)=\psi_{0}
\end{array}\right.
$$


The linearized system around $(\psi, u)$ is

$$
\left(\Sigma_{l}\right)\left\{\begin{array}{l}
i \dot{\Psi}=-\Psi^{\prime \prime}+\left(\dot{u}-4 u^{2}\right)(t) x^{2} \Psi+(\dot{v}-8 u v)(t) x^{2} \psi, x \in(0,1), t \in(0, T), \\
\Psi(t, 0)=\Psi(t, 1)=0 .
\end{array}\right.
$$

Let us introduce the distances, for $s=2,4,6,8$,

$$
\delta_{s}:=\left\|\left(\psi_{0}, u\right)-\left(\psi_{r e f}(0), 0\right)\right\|_{E_{s}}
$$

We want to prove that, there exists a constant $C$ such that, when $\delta_{4}$ is small enough, then, for every $\Psi_{f} \in$ $H_{(0)}^{7}((0,1), \mathbb{C})$ there exists a trajectory $(\Psi, v)$ of $\left(\Sigma_{l}\right)$ with $\Psi(0)=0, \Psi(T)=\Psi_{f}, v \in \overline{H_{0}^{3}}((0, T), \mathbb{C})$,

$$
\|v\|_{H_{0}^{1}((0, T), \mathbb{R})} \leqslant C\left\|\Psi_{f}\right\|_{H^{3}}, \quad \text { and } \quad\|v\|_{H_{0}^{3}((0, T), \mathbb{R})} \leqslant C\left[\left\|\Psi_{f}\right\|_{H^{7}}+\delta_{8}\left\|\Psi_{f}\right\|_{H^{3}}\right]
$$

In order to solve this problem, one transforms the controllability condition $\Psi(T)=\Psi_{f}$ into a moment problem on the control $v$. For technical reasons explained in Remark 8, we don't decompose the solution $\Psi$ of $\left(\Sigma_{l}\right)$ on the fixed basis $\left(\varphi_{k}\right)_{k \in \mathbb{N}^{*}}$ as in Section 2 but on a moving basis.

For $\gamma \in \mathbb{R}$, we introduce the operator $A_{\gamma}$ defined by

$$
D\left(A_{\gamma}\right):=H_{(0)}^{2}((0,1), \mathbb{C}), \quad A_{\gamma} \varphi:=-\varphi^{\prime \prime}+\gamma x^{2} \varphi
$$

Let $\left(\lambda_{k, \gamma}\right)_{k \in \mathbb{N}^{*}}$ be the non decreasing sequence of its eigenvalues (written as many times as their multiplicity) and $\left(\varphi_{k, \gamma}\right)_{k \in \mathbb{N}^{*}}$ associated eigenvectors, which form an orthonormal basis of $L^{2}((0,1), \mathbb{C})$. The maps $\gamma \mapsto \lambda_{k, \gamma}$ and $\gamma \mapsto \varphi_{k, \gamma}$ are analytic, which gives a sense to the notations

$$
\left.\lambda_{k, \gamma_{1}}^{\prime} \quad \text { and } \quad \frac{\mathrm{d} \varphi_{k, \gamma}}{\mathrm{d} \gamma}\right]_{\gamma_{1}}
$$

Let $\mu:=\dot{u}-4 u^{2}$. We consider the decomposition

$$
\Psi(t)=\sum_{k=1}^{\infty} y_{k}(t) \xi_{k}(t)
$$

where

$$
\begin{gathered}
\xi_{1}(t):=\psi(t), \quad \xi_{k}(t):=\varphi_{k, \mu(t)}-\left\langle\varphi_{k, \mu(t)}, \psi(t)\right\rangle \psi(t), \text { when } k \geqslant 2, \\
y_{1}(t):=\langle\Psi(t), \psi(t)\rangle, \quad y_{k}(t):=\left\langle\Psi(t), \varphi_{k, \mu(t)}\right\rangle-\frac{\left\langle\Psi(t), \varphi_{1, \mu(t)}\right\rangle}{\left\langle\psi(t), \varphi_{1, \mu(t)}\right\rangle}\left\langle\psi(t), \varphi_{k, \mu(t)}\right\rangle, \text { when } k \geqslant 2 .
\end{gathered}
$$

The partial differential equation satisfied by $\Psi$ provides an ordinary differential equation for each components $y_{k}(t)$, that can be solved. Then the equality $\Psi(T)=\Psi_{f}$ is equivalent to the equality $M_{\left(\psi_{0}, u\right)}(v)=d$ where $d=\left(d_{k}\right)_{k \in \mathbb{N}^{*}}, M_{\left(\psi_{0}, u\right)}(v)=\left(M_{\left(\psi_{0}, u\right)}(v)_{k}\right)_{k \in \mathbb{N}^{*}}$ and

$$
\begin{gathered}
M_{\left(\psi_{0}, u\right)}(v)_{1}:=-i \int_{0}^{T}(\dot{v}-8 u v)\left\langle x^{2} \psi, \psi\right\rangle \mathrm{d} t, \\
M_{\left(\psi_{0}, u\right)}(v)_{k}:=\int_{0}^{T}\left[-i(\dot{v}-8 u v)\left\langle x^{2} \psi, \varphi_{k, \mu}\right\rangle-\dot{\alpha}\left\langle\psi, \varphi_{k, \mu}\right\rangle+\dot{\mu}\left(\left\langle\Psi, \frac{\mathrm{d} \varphi_{k, \gamma}}{\mathrm{d} \gamma}\right]_{\mu}\right\rangle\right. \\
\left.\left.\left.-\alpha\left\langle\psi, \frac{\varphi_{k, \gamma}}{d \gamma}\right]_{\mu}\right\rangle\right)\right] \mathrm{e}^{i \int_{0}^{t} \lambda_{k, \mu(s)} \mathrm{d} s} \mathrm{~d} t, \text { for every } k \geqslant 2,
\end{gathered}
$$


where

and

$$
\alpha(t):=\frac{\left\langle\Psi(t), \varphi_{1, \mu(t)}\right\rangle}{\left\langle\psi(t), \varphi_{1, \mu(t)}\right\rangle}
$$

$$
\begin{gathered}
d_{1}:=\left\langle\Psi_{f}, \psi(T)\right\rangle, \\
d_{k}:=\left(\left\langle\Psi_{f}, \varphi_{k}\right\rangle-\frac{\left\langle\Psi_{f}, \varphi_{1}\right\rangle}{\left\langle\psi(T), \varphi_{1}\right\rangle}\left\langle\psi(T), \varphi_{k}\right\rangle\right) \mathrm{e}^{i \int_{0}^{T} \lambda_{k, \mu(s)} \mathrm{d} s}, \text { for every } k \geqslant 2 .
\end{gathered}
$$

In order to prove the surjectivity of $M_{\left(\psi_{0}, u\right)}$ when $\delta_{4}$ is small, we use the surjectivity of $M_{\left(\psi_{r e f}(0), 0\right)}$ thanks to the following proposition.

Proposition 6. Let $\mathcal{M}$ and $\widetilde{\mathcal{M}}$ be continuous linear maps from $\overline{H_{0}^{1}}((0, T), \mathbb{R})$ to $h^{3}\left(\mathbb{N}^{*}, \mathbb{C}\right)$, from $\overline{H_{0}^{2}}((0, T), \mathbb{R})$ to $h^{5}\left(\mathbb{N}^{*}, \mathbb{C}\right)$ and from $\overline{H_{0}^{3}}((0, T), \mathbb{R})$ to $h^{7}\left(\mathbb{N}^{*}, \mathbb{C}\right)$. We assume that there exists a positive constant $C_{0}$, such that $\widetilde{\mathcal{M}}$ has a right inverse

$$
\widetilde{M}^{-1}: h^{7}\left(\mathbb{N}^{*}, \mathbb{C}\right) \rightarrow \overline{H_{0}^{3}}((0, T), \mathbb{R})
$$

which satisfies, for every $d \in h^{7}\left(\mathbb{N}^{*}, \mathbb{C}\right)$,

$$
\left\|\widetilde{\mathcal{M}}^{-1}(d)\right\|_{E} \leqslant C_{0}\|d\|_{F}
$$

for every $(E, F) \in\left\{\left(\overline{H_{0}^{1}}, h^{3}\right),\left(\overline{H_{0}^{2}}, h^{5}\right),\left(\overline{H_{0}^{3}}, h^{7}\right)\right\}$. We also assume that there exist $\mathcal{C}_{0}, \mathcal{C}_{1}, \mathcal{C}_{2}>0$, such that, for every $v \in H_{0}^{2}((0, T), \mathbb{R})$,

$$
\begin{aligned}
& \|(\widetilde{\mathcal{M}}-\mathcal{M})(v)\|_{h^{3}\left(\mathbb{N}^{*}, \mathbb{C}\right)} \leqslant \mathcal{C}_{0}\|v\|_{H_{0}^{1}((0, T), \mathbb{R})}, \\
& \|(\widetilde{\mathcal{M}}-\mathcal{M})(v)\|_{h^{5}\left(\mathbb{N}^{*}, \mathbb{C}\right)} \leqslant \mathcal{C}_{0}\|v\|_{H_{0}^{2}((0, T), \mathbb{R})}+\mathcal{C}_{1}\|v\|_{H_{0}^{1}((0, T), \mathbb{R})}, \\
& \|(\widetilde{\mathcal{M}}-\mathcal{M})(v)\|_{h^{7}\left(\mathbb{N}^{*}, \mathbb{C}\right)} \leqslant \mathcal{C}_{0}\|v\|_{H_{0}^{3}((0, T), \mathbb{R})}+\mathcal{C}_{1}\|v\|_{H_{0}^{2}((0, T), \mathbb{R})}+\mathcal{C}_{2}\|v\|_{H_{0}^{1}((0, T), \mathbb{R})} .
\end{aligned}
$$

We assume $C_{0} \mathcal{C}_{0}<1$. Then $\mathcal{M}$ has a right inverse

$$
\mathcal{M}^{-1}: h^{7}\left(\mathbb{N}^{*}, \mathbb{C}\right) \rightarrow \overline{H_{0}^{3}}((0, T), \mathbb{R}),
$$

which satisfies, for every $d \in h^{7}(\mathbb{N}, \mathbb{C})$,

$$
\begin{aligned}
& \left\|\mathcal{M}^{-1}(d)\right\|_{H_{0}^{1}((0, T), \mathbb{R})} \leqslant X\|d\|_{h^{3}\left(\mathbb{N}^{*}, \mathbb{C}\right)}, \\
& \left\|\mathcal{M}^{-1}(d)\right\|_{H_{0}^{2}((0, T), \mathbb{R})} \leqslant X\|d\|_{h^{5}\left(\mathbb{N}^{*}, \mathbb{C}\right)}+Y\|d\|_{h^{3}\left(\mathbb{N}^{*}, \mathbb{C}\right)}, \\
& \left\|\mathcal{M}^{-1}(d)\right\|_{H_{0}^{3}((0, T), \mathbb{R})} \leqslant X\|d\|_{h^{7}\left(\mathbb{N}^{*}, \mathbb{C}\right)}+Y\|d\|_{h^{5}\left(\mathbb{N}^{*}, \mathbb{C}\right)}+Z\|d\|_{h^{3}\left(\mathbb{N}^{*}, \mathbb{C}\right)}
\end{aligned}
$$

where

$$
X:=\frac{C_{0}}{1-C_{0} \mathcal{C}_{0}}, Y:=\frac{C_{0}^{2} \mathcal{C}_{1}}{\left(1-C_{0} \mathcal{C}_{0}\right)^{2}}, Z:=\frac{C_{0}^{2} \mathcal{C}_{1}^{2}}{\left(1-C_{0} \mathcal{C}_{0}\right)^{3}}+\frac{C_{0}^{2} \mathcal{C}_{2}}{\left(1-C_{0} \mathcal{C}_{0}\right)^{2}} .
$$

Proof. Let $d \in h^{7}\left(\mathbb{N}^{*}, \mathbb{C}\right)$. We define by induction the sequence $\left(w_{n}\right)_{n \in \mathbb{N}}$ in $\overline{H_{0}^{3}}((0, T), \mathbb{R})$ by

$$
\left\{\begin{array}{l}
w_{0}:=\widetilde{\mathcal{M}}^{-1}(d), \\
w_{n+1}:=\widetilde{\mathcal{M}}^{-1}\left[(\widetilde{\mathcal{M}}-\mathcal{M})\left(w_{n}\right)\right] .
\end{array}\right.
$$

The function $w:=\sum_{n=0}^{\infty} w_{n}$ gives a suitable candidate for $\mathcal{M}^{-1}(d)$. 
Remark 8. If we had decomposed $\Psi$ on the fixed basis $\left(\varphi_{k}\right), \Psi(t)=\sum_{k=1}^{\infty} y_{k}(t) \varphi_{k}$, we would have got

$$
y_{k}(T)=\left(\int_{0}^{T}-i\left[\mu(t)\left\langle x^{2} \Psi(t), \varphi_{k}\right\rangle+(\dot{v}-8 u v)(t)\left\langle x^{2} \psi(t), \varphi_{k}\right\rangle\right] \mathrm{e}^{i \lambda_{k} t} \mathrm{~d} t\right) \mathrm{e}^{-i \lambda_{k} T}
$$

and the sequence

$$
\left(\int_{0}^{T} \mu(t)\left\langle x^{2} \Psi(t), \varphi_{k}\right\rangle \mathrm{e}^{i \lambda_{k} t}\right)_{k \in \mathbb{N}^{*}}
$$

does not belong to $h^{3}\left(\mathbb{N}^{*}, \mathbb{C}\right)$ when $v$ is only in $\overline{H_{0}^{1}}((0, T), \mathbb{R})$. By decomposing $\Psi$ on a moving basis, $\Psi(t)=$ $\sum_{k=1}^{\infty} z_{k}(t) \varphi_{k, u(t)}$, we make this term disappear, indeed

$$
\left.z_{k}(T)=\left(\int_{0}^{T}\left[-i(\dot{v}-8 u v)(t)\left\langle x^{2} \psi(t), \varphi_{k, \mu(t)}\right\rangle+\dot{\mu}(t)\left\langle\Psi(t), \frac{\mathrm{d} \varphi_{k, \gamma}}{\mathrm{d} \gamma}\right]_{\mu(t)}\right\rangle\right] \mathrm{e}^{i \int_{0}^{t} \lambda_{k, u(s)} \mathrm{d} s} \mathrm{~d} t\right) \mathrm{e}^{-i \int_{0}^{T} \lambda_{k, u(s)} \mathrm{d} s} .
$$

and the new term belongs to $h^{3}\left(\mathbb{N}^{*}, \mathbb{C}\right)$ when $v$ is only $H^{1}((0, T), \mathbb{C})$. Since we want to use differences of linear maps the type $M_{\left(\psi_{0}, u\right)}-M_{\left(\psi^{r e f}(0), 0\right)}$ (each one corresponds to the control of a linearized system), as in Proposition 6, we need to use linear maps with images in the same space. This is why we use the basis $\left(\xi_{k}(t)\right)$ instead of $\left(\varphi_{k, u(t)}\right)$ : the condition $\Psi_{f} \in T_{\mathcal{S}}(\psi(T))$ corresponds to $\left\langle\Psi(t), \xi_{1}(t)\right\rangle \in i \mathbb{R}$.

In Section 4.4.2, we prove the following proposition

Proposition 7. There exists a constant $C>0$ such that, when $\delta_{4}$ is small enough, for every $v \in \overline{H_{0}^{3}}((0, T), \mathbb{R})$, we have

$$
\begin{aligned}
& \left\|\left(M_{\left(\psi_{0}, u\right)}-M_{\left(\psi_{r e f}(0), 0\right)}\right)(v)\right\|_{h^{3}\left(\mathbb{N}^{*}, \mathbb{C}\right)} \leqslant C \delta_{4}\|v\|_{H^{1}}, \\
& \left\|\left(M_{\left(\psi_{0}, u\right)}-M_{\left(\psi_{r e f}(0), 0\right)}\right)(v)\right\|_{h^{5}\left(\mathbb{N}^{*}, \mathbb{C}\right)} \leqslant C\left[\delta_{4}\|v\|_{H^{2}}+\delta_{6}\|v\|_{H^{1}}\right], \\
& \left\|\left(M_{\left(\psi_{0}, u\right)}-M_{\left(\psi_{r e f}(0), 0\right)}\right)(v)\right\|_{h^{7}\left(\mathbb{N}^{*}, \mathbb{C}\right)} \leqslant C\left[\delta_{4}\|v\|_{H^{3}}+\delta_{6}\|v\|_{H^{2}}+\delta_{8}\|v\|_{H^{1}}\right] .
\end{aligned}
$$

For the proof of this proposition, we need few technical results stated in the next section.

\subsubsection{Preliminaries}

In this section, we use the bounds proved in Appendix A. The constants $\gamma^{*}$ and $C^{*}$ are such that all the propositions of Appendix A are true. Let $T>0$. For $\mu \in C^{0}\left((0, T),\left(-\gamma^{*}, \gamma^{*}\right)\right), w \in L^{2}((0, T), \mathbb{R})$ and $f \in C^{0}\left([0, T], L^{2}((0,1), \mathbb{R})\right)$, we consider the sequences

$$
\begin{gathered}
S_{0}:=\left(\int_{0}^{T} w(t)\left\langle f(t), \varphi_{k, \mu(t)}\right\rangle \mathrm{e}^{i \int_{0}^{t} \lambda_{k, \mu(s)} \mathrm{d} s} \mathrm{~d} t\right)_{k \in \mathbb{N}^{*}}, \\
\left.S_{1}:=\left(\int_{0}^{T} w(t)\left\langle f(t), \frac{\mathrm{d} \varphi_{k, \gamma}}{\mathrm{d} \gamma}\right]_{\mu(t)}\right\rangle \mathrm{e}^{i \int_{0}^{t} \lambda_{k, \mu(s)} \mathrm{d} s} \mathrm{~d} t\right)_{k \in \mathbb{N}^{*}} .
\end{gathered}
$$

Lemma 1. There exists $\gamma^{*}>0, C^{*}>0$ such that, for every $\gamma_{1} \in\left(-\gamma^{*}, \gamma^{*}\right)$, for every $f \in L^{2}((0,1), \mathbb{R})$,

$$
\left.\sum_{k=1}^{\infty}\left|k\left\langle f, \frac{\mathrm{d} \varphi_{k, \gamma}}{\mathrm{d} \gamma}\right]_{\gamma_{1}}\right\rangle\right|^{2} \leqslant C\|f\|_{L^{2}}^{2}
$$

and for every $f \in H^{2} \cap H_{0}^{1}((0,1), \mathbb{R})$,

$$
\left.\sum_{k=1}^{\infty}\left|k^{3}\left\langle f, \frac{\mathrm{d} \varphi_{k, \gamma}}{\mathrm{d} \gamma}\right]_{\gamma_{1}}\right\rangle\right|^{2} \leqslant C\|f\|_{H^{2}}^{2}
$$


Proof. Thanks to (A.1), we have, for $f \in L^{2}((0,1), \mathbb{R})$,

$$
\left.\sum_{k=1}^{\infty}\left|k\left\langle f, \frac{\mathrm{d} \varphi_{k, \gamma}}{\mathrm{d} \gamma}\right]_{0}\right\rangle\right|^{2}=\sum_{k=1}^{\infty}\left|\sum_{j=1}^{\infty} a_{k, j}\left\langle f, \varphi_{j}\right\rangle\right|^{2}
$$

where $a_{k, j}:=k x_{k, j}$ when $k \neq j$ and $a_{k, k}=0$. We check the existence of $C>0$ such that,

$$
\forall j \in \mathbb{N}^{*}, \sum_{k=1}^{\infty}\left|a_{k, j}\right| \leqslant C \quad \text { and } \quad \forall k \in \mathbb{N}^{*}, \sum_{j=1}^{\infty}\left|a_{k, j}\right| \leqslant C .
$$

Thus, the Cauchy-Schwarz inequality justifies that,

$$
\forall\left(x_{j}\right)_{j \in \mathbb{N}^{*}} \in l^{2}\left(\mathbb{N}^{*}, \mathbb{C}\right), \quad \sum_{k=1}^{\infty}\left|\sum_{j=1}^{\infty} a_{k, j} x_{j}\right|^{2} \leqslant C^{2} \sum_{j=1}^{\infty}\left|x_{j}\right|^{2},
$$

which gives the conclusion for $\gamma_{1}=0$. For $\gamma_{1} \neq 0$, we conclude thanks to the result for $\gamma_{1}=0$ and (A.8). For $f \in H^{2} \cap H_{0}^{1}((0,1), \mathbb{C})$, we use integrations by parts and the equation (A.7).

Proposition 8. Let $T>0$. There exists $C>0$ such that, for every $\mu \in H^{1}((0, T), \mathbb{R})$ with $\|\mu\|_{H^{1}((0, T), \mathbb{R})} \leqslant 1$,

- when $w \in L^{2}((0, T), \mathbb{R})$ and $f \in C^{0}\left([0, T], H^{3} \cap H_{0}^{1}((0,1), \mathbb{R})\right)$, then $S_{0} \in h^{3}\left(\mathbb{N}^{*}, \mathbb{C}\right)$ and

$$
\left\|S_{0}\right\|_{h^{3}\left(\mathbb{N}^{*}, \mathbb{C}\right)} \leqslant C\|w\|_{L^{2}}\|f\|_{C^{0}\left([0, T], H^{3}\right)},
$$

more precisely,

$$
\begin{aligned}
S_{0}= & \left(\frac{1}{\lambda_{k}} \int_{0}^{T} w(t)\left\langle A f(t), \varphi_{k}\right\rangle \mathrm{e}^{i \lambda_{k} t} \mathrm{~d} t\right)_{k \in \mathbb{N}^{*}} \\
& + \text { terms with an } h^{3} \text { norm bounded by } C\|\mu\|_{H^{1}}\|w\|_{L^{2}}\|f\|_{C^{0}\left([0, T], H^{3}\right)}
\end{aligned}
$$

- when $w \in H_{0}^{1}((0, T), \mathbb{R})$ and $f \in C^{1}\left([0, T], H^{3} \cap H_{0}^{1}((0,1), \mathbb{R})\right.$ then $S_{0} \in h^{5}\left(\mathbb{N}^{*}, \mathbb{C}\right)$ and

$$
\left\|S_{0}\right\|_{h^{5}\left(\left(\mathbb{N}^{*}, \mathbb{C}\right)\right.} \leqslant C\left[\|w\|_{H_{0}^{1}}\|f\|_{C^{0}\left([0, T], H^{3}\right)}+\|w\|_{L^{2}}\|f\|_{C^{1}\left([0, T], H^{3}\right)}\right],
$$

more precisely

$$
S_{0}=\left(-\frac{1}{i \lambda_{k}} \int_{0}^{T}\left(\dot{w}(t)\left\langle f(t), \varphi_{k}\right\rangle+w(t)\left\langle\dot{f}, \varphi_{k}\right\rangle\right) \mathrm{e}^{i \lambda_{k} t} \mathrm{~d} t\right)_{k \in \mathbb{N}^{*}}
$$

+ terms with an $h^{5}$ norm bounded by $C\|\mu\|_{H^{1}}\left[\|w\|_{H^{1}}\|f\|_{C^{0}\left([0, T], H^{3}\right)}+\|w\|_{L^{2}}\|f\|_{C^{1}\left([0, T], H^{3}\right)}\right]$;

- when $\mu \in H^{2}((0, T), \mathbb{R}), w \in H_{0}^{2}((0, T), \mathbb{R})$ and $f \in C^{3}\left([0, T], H^{3} \cap H_{0}^{1}((0,1), \mathbb{R})\right.$ then $S_{0} \in h^{7}\left(\mathbb{N}^{*}, \mathbb{C}\right)$ and

$$
\begin{aligned}
\left\|S_{0}\right\|_{h^{7}\left(\left(\mathbb{N}^{*}, \mathbb{C}\right)\right.} \leqslant & C\left\{\|w\|_{H_{0}^{2}}\|f\|_{C^{0}\left([0, T], H^{3}\right)}+\|w\|_{H^{1}}\left[\|f\|_{C^{1}\left([0, T], H^{3}\right)}+\|\mu\|_{H^{2}}\|f\|_{C^{0}\left([0, T], H^{2}\right)}\right]\right. \\
& \left.+\|w\|_{L^{2}}\|f\|_{C^{2}\left([0, T], H^{3}\right)}\right\}
\end{aligned}
$$

more precisely,

$$
\begin{aligned}
S_{0}= & \left(-\frac{1}{i \lambda_{k}} \int_{0}^{T}\left(\ddot{w}(t)\left\langle f(t), \varphi_{k}\right\rangle+2 \dot{w}(t)\left\langle\dot{f}, \varphi_{k}\right\rangle+w(t)\left\langle\ddot{f}, \varphi_{k}\right\rangle\right) \mathrm{e}^{i \lambda_{k} t} \mathrm{~d} t\right)_{k \in \mathbb{N}^{*}} \\
& + \text { terms with an } h^{7} \text { norm bounded by } \\
& C\left\{\|\mu\|_{H^{1}}\left[\|w\|_{H^{2}}\|f\|_{C^{0}\left([0, T], H^{3}\right)}+\|w\|_{H^{1}}\|f\|_{C^{1}\left([0, T], H^{3}\right)}+\|w\|_{L^{2}}\|f\|_{C^{2}\left([0, T], H^{3}\right)}\right]\right. \\
& \left.+\mid \mu\left\|_{H^{2}}\right\| w\left\|_{H^{1}}\right\| f \|_{C^{0}\left([0, T], H^{2}\right)}\right\}
\end{aligned}
$$


Proposition 9. Let $T>0$. There exists $C>0$ such that, for every $\mu \in H^{1}((0, T), \mathbb{R})$ with $\|\mu\|_{H^{1}((0, T), \mathbb{R})} \leqslant 1$,

- when $w \in L^{2}((0, T), \mathbb{R})$ and $f \in C^{0}\left([0, T], H^{2} \cap H_{0}^{1}((0,1), \mathbb{R})\right)$, then $S_{1} \in h^{3}\left(\mathbb{N}^{*}, \mathbb{C}\right)$ and

$$
\left\|S_{1}\right\|_{h^{3}\left(\mathbb{N}^{*}, \mathbb{C}\right)} \leqslant C\|w\|_{L^{2}}\|f\|_{C^{0}\left([0, T], H^{2}\right)}
$$

- when $w \in H_{0}^{1}((0, T), \mathbb{R})$ and $f \in C^{1}\left([0, T], H^{2} \cap H_{0}^{1}((0,1), \mathbb{R})\right.$ then $S_{1} \in h^{5}\left(\mathbb{N}^{*}, \mathbb{C}\right)$ and

$$
\left\|S_{1}\right\|_{h^{5}\left(\left(\mathbb{N}^{*}, \mathbb{C}\right)\right.} \leqslant C\left[\|w\|_{H^{1}}\|f\|_{C^{0}\left([0, T], H^{2}\right)}+\|w\|_{L^{2}}\|f\|_{C^{1}\left([0, T], H^{2}\right)}\right] ;
$$

- when $\mu \in H^{2}((0, T), \mathbb{R}), w \in H_{0}^{2}((0, T), \mathbb{R})$ and $f \in C^{3}\left([0, T], H^{2} \cap H_{0}^{1}((0,1), \mathbb{R})\right.$ then $S_{1} \in h^{7}\left(\mathbb{N}^{*}, \mathbb{C}\right)$ and

$$
\left\|S_{1}\right\|_{h^{7}\left(\left(\mathbb{N}^{*}, \mathbb{C}\right)\right.} \leqslant C\left[\|w\|_{H_{0}^{2}}\|f\|_{C^{0}\left([0, T], H^{2}\right)}+\|w\|_{H^{1}}\|f\|_{C^{1}\left([0, T], H^{2}\right)}+\|w\|_{L^{2}}\|f\|_{C^{2}\left([0, T], H^{2}\right)}\right] .
$$

In the end of this section, we justify the $h^{3}$-bound on $S_{1}$ and all the bounds on $S_{0}$. The other bounds can be proved in the same way.

Remark 9. Propositions 8 and 9 hold in any positive time $T$. Thus, if the linearized system around $\left(\psi_{r e f}, u \equiv 0\right)$ is controllable in time $T$ with the bounds (3.8) and (3.9) (corresponding to $v=0$ ), then, the nonlinear system is locally controllable in the same time $T$, whatever the value of $T$ is.

Proof of the $h^{3}$-bounds of Proposition 9. Thanks to Cauchy-Schwarz inequality and Lemma 1, we have

$$
\left\|S_{1}\right\|_{h^{3}}^{2} \leqslant\left.\int_{0}^{T}|w(t)| 2 \mathrm{~d} t \int_{0}^{T} \sum_{k=1}^{\infty}\left|k^{3}\left\langle f(t), \frac{\mathrm{d} \varphi_{k, \gamma}}{\mathrm{d} \gamma}\right]_{\mu(t)}\right\rangle\right|^{2} \mathrm{~d} t \leqslant C\|w\|_{L^{2}}^{2}\|f\|_{C^{0}\left([0, T], H^{2}\right.}^{2} .
$$

Proof. of the $h^{3}$-bounds of Proposition 8. First, we prove that, when $f \in C^{0}\left([0, T], H^{1}\right)$ and $\mu \equiv 0$ then $S_{0} \in h^{1}\left(\mathbb{N}^{*}, \mathbb{C}\right)$. Indeed, we have

$$
S_{0, k}=\int_{0}^{T} w(t)\left\langle g(t), \varphi_{k}\right\rangle \mathrm{e}^{i \lambda_{k} t} \mathrm{~d} t+\frac{\sqrt{2}}{k \pi}\left((-1)^{k+1} \int_{0}^{T} w(t) f(t, 1) \mathrm{e}^{i \lambda_{k} t} \mathrm{~d} t+\int_{0}^{T} w(t) f(t, 0) \mathrm{e}^{i \lambda_{k} t} \mathrm{~d} t\right),
$$

where $g(t, x):=f(t, x)-f(t, 1) x-f(t, 0)(1-x)$. Since $g \in C^{0}\left([0, T], H_{0}^{1}((0,1), \mathbb{C})\right)$, then, for every $t$, $\left(\left\langle g(t), \varphi_{k}\right\rangle\right)_{k \in \mathbb{N}^{*}}$ belongs to $h^{1}\left(\mathbb{N}^{*}, \mathbb{C}\right)$. When $T \in(2 / \pi) \mathbb{N}$, we can conclude thanks to the Bessel Parseval inequality in $L^{2}((0, T), \mathbb{C})$. When $T \notin(2 / \pi) \mathbb{N}$, we use the following consequence of the Ingham inequality.

Lemma 2. Let $T>0$. There exists $C>0$ such that, for every $g \in L^{2}((0, T), \mathbb{C})$,

$$
\left(\sum_{k=1}^{\infty}\left|c_{k}\right|^{2}\right)^{1 / 2} \leqslant C\|g\|_{L^{2}(0, T)} \text { where } c_{k}:=\int_{0}^{T} g(t) \mathrm{e}^{i \lambda_{k} t} \mathrm{~d} t
$$

Proof of Lemma 2. We know that there exists a constant $C>0$ such that, for every $N \in \mathbb{N}$, and for every $\left(a_{k}\right)_{1 \leqslant k \leqslant N} \subset \mathbb{C}$,

$$
\left\|\sum_{k=1}^{N} a_{k} \mathrm{e}^{-i \lambda_{k} t}\right\|_{L^{2}(0, T)}^{2} \leqslant C \sum_{k=1}^{N}\left|a_{k}\right|^{2}
$$

(see [25], Th. 4). Let us introduce the following closed subspace of $L^{2}((0, T), \mathbb{C}), \mathcal{V}:=\overline{\operatorname{Span}\left\{\mathrm{e}^{-i \lambda_{k} t}, k \in \mathbb{N}\right\}}$. The family $\left(\mathrm{e}^{-i \lambda_{k} t}\right)_{k \in \mathbb{N}^{*}}$ is minimal in $L^{2}((0, T), \mathbb{C})$ because it satisfies an Ingham inequality (see [30], Lem. 1.2.7). 
Thus, there exists a biorthonormal family $\left(z_{k}\right)_{k \in \mathbb{N}^{*}} \subset \mathcal{V}$ (see [30], Th. 1.2.5): $\left\langle\mathrm{e}^{-i \lambda_{k} t}, z_{j}\right\rangle=\delta_{j, k}$. Let $g \in$ $L^{2}((0, T), \mathbb{C})$ and $g_{1}$ be the orthogonal projection in $L^{2}((0, T), \mathbb{C})$ of $g$ on $\mathcal{V}, g_{1}=\sum_{k=1}^{\infty} c_{k} z_{k}$. We have

$$
\begin{aligned}
\left\|c_{k}\right\|_{l^{2}} & =\sup \left\{\left|\sum_{k=1}^{\infty} c_{k} \overline{a_{k}}\right| ; a=\left(a_{k}\right)_{k \in \mathbb{N}} \in l^{2}(\mathbb{N}, \mathbb{C}),\|a\|_{l^{2}} \leqslant 1\right\} \\
& =\sup \left\{\left|\sum_{k=1}^{N} c_{k} \overline{a_{k}}\right| ; N \in \mathbb{N}^{*},\left(a_{k}\right)_{1 \leqslant k \leqslant N} \subset \mathbb{C}, \sum_{k=1}^{N}\left|a_{k}\right|^{2} \leqslant 1\right\} \\
& =\sup \left\{\left|\int_{0}^{T} g_{1}(t) \overline{h(t)} \mathrm{d} t\right| ; N \in \mathbb{N}^{*}, h=\sum_{k=1}^{N} a_{k} \mathrm{e}^{-i \lambda_{k} t},\left(a_{k}\right)_{1 \leqslant k \leqslant N} \subset \mathbb{C}, \sum_{k=1}^{N}\left|a_{k}\right|^{2} \leqslant 1\right\} \\
& \leqslant C\left\|g_{1}\right\|_{L^{2}} \leqslant C\|g\|_{L^{2}} .
\end{aligned}
$$

Now, let $f \in C^{0}\left([0, T], H^{3} \cap H_{0}^{1}((0,1), \mathbb{C})\right)$. Let us consider the decomposition

$$
\begin{aligned}
S_{0, k}= & \int_{0}^{T} w(t)\left(\frac{1}{\lambda_{k, \mu(t)}}-\frac{1}{\lambda_{k}}\right)\left\langle A_{\mu(t)} f(t), \varphi_{k, \mu(t)}\right\rangle \mathrm{e}^{i \int_{0}^{t} \lambda_{k, \mu(s)} \mathrm{d} s} \mathrm{~d} t \\
& +\frac{1}{\lambda_{k}} \int_{0}^{T} w(t)\left\langle A_{\mu(t)} f(t), \varphi_{k, \mu(t)}-\varphi_{k}-\mu(t) \varphi_{k}^{(1)}\right\rangle \mathrm{e}^{i \int_{0}^{t} \lambda_{k, \mu(s)} \mathrm{d} s} \mathrm{~d} t \\
& +\frac{1}{\lambda_{k}} \int_{0}^{T} w(t) \mu(t)\left\langle A_{\mu(t)} f(t), \varphi_{k}^{(1)}\right\rangle \mathrm{e}^{i \int_{0}^{t} \lambda_{k, \mu(s)} d s} \mathrm{~d} t \\
& +\frac{1}{\lambda_{k}} \int_{0}^{T} w(t)\left\langle A_{\mu(t)} f(t), \varphi_{k}\right\rangle\left(\mathrm{e}^{i \int_{0}^{t} \lambda_{k, \mu(s)} d s}-\mathrm{e}^{i \lambda_{k} t}\right) \mathrm{d} t \\
& +\frac{1}{\lambda_{k}} \int_{0}^{T} w(t)\left\langle A_{\mu(t)} f(t), \varphi_{k}\right\rangle \mathrm{e}^{i \lambda_{k} t} \mathrm{~d} t
\end{aligned}
$$

called

$$
S_{0}=S_{0, a}+S_{0, b}+S_{0, c}+S_{0, d}+S_{0, e} .
$$

The first part of this proof justifies that

$$
\left\|S_{0, e}\right\|_{h^{3}} \leqslant C\|w\|_{L^{2}}\|f\|_{C^{0}\left([0, T], H^{3}\right)} .
$$

Thanks to (A.4) and the Cauchy-Schwarz inequality in $L^{2}((0, T), \mathbb{R})$, we get

$$
\left\|S_{0, a}\right\|_{h^{3}} \leqslant C\|\mu\|_{H^{1}}\|w\|_{L^{2}}\left\|A_{\mu} f\right\|_{C^{0}\left([0, T], L^{2}\right)} \leqslant C\|\mu\|_{H^{1}}\|w\|_{L^{2}}\|f\|_{C^{0}\left([0, T], H^{2}\right)} .
$$

Thanks to (A.10), we get,

$$
\left\|S_{0, b}\right\|_{h^{3}} \leqslant C\|\mu\|_{H^{1}}^{2}\|w\|_{L^{2}}\left\|A_{\mu} f\right\|_{C^{0}\left([0, T], L^{2}\right)} \leqslant C\|\mu\|_{H^{1}}\|w\|_{L^{2}}\|f\|_{C^{0}\left([0, T], H^{2}\right)} .
$$

In the same way and thanks to Lemma 1 , we get

$$
\left\|S_{0, c}\right\|_{h^{3}} \leqslant C\|w \mu\|_{L^{2}}\left\|A_{\mu} f\right\|_{C^{0}\left([0, T], L^{2}\right)} \leqslant C\|\mu\|_{H^{1}}\|w\|_{L^{2}}\|f\|_{C^{0}\left([0, T], H^{2}\right)} .
$$

Thanks to (A.12), we can write

$$
\int_{0}^{t} \lambda_{k, \mu(s)} \mathrm{d} s=\lambda_{k} t+\frac{1}{3} \int_{0}^{t} \mu(s) \mathrm{d} s+c_{k}(t) \text { where }\left|c_{k}(t)\right| \leqslant \frac{C\|\mu\|_{H^{1}}}{k^{2}} .
$$


Thus, we have the decomposition

$$
\begin{aligned}
S_{0, d, k}= & \frac{1}{\lambda_{k}} \int_{0}^{T} w(t)\left\langle A_{\mu(t)} f(t), \varphi_{k}\right\rangle\left(\mathrm{e}^{i \int_{0}^{t} \lambda_{k, \mu(s)} \mathrm{d} s}-\mathrm{e}^{i\left(\lambda_{k} t+\frac{1}{3} \int_{0}^{t} \mu(s) \mathrm{d} s\right)}\right) \mathrm{d} t \\
& +\frac{1}{\lambda_{k}} \int_{0}^{T} w(t)\left(\mathrm{e}^{i \frac{1}{3} \int_{0}^{t} \mu(s) \mathrm{d} s}-1\right)\left\langle A_{\mu(t)} f(t), \varphi_{k}\right\rangle \mathrm{e}^{i \lambda_{k} t} \mathrm{~d} t .
\end{aligned}
$$

The bound (4.6) justifies that the $h^{3}$-norm of the first term of this decomposition is bounded by $C\|\mu\|_{H^{1}}\|w\|_{L^{2}}\|f\|_{C^{0}\left([0, T], H^{2}\right)}$. Using the first par of this proof, we get the following bound for the $h^{3}$-norm of the second term of this decomposition

$$
C\left\|w(t)\left(1-\mathrm{e}^{i \frac{1}{3} \int_{0}^{t} \mu(s) \mathrm{d} s}\right)\right\|_{L^{2}}\left\|A_{\mu} f\right\|_{C^{0}\left([0, T], H^{1}\right)} \leqslant C\|\mu\|_{H^{1}}\|w\|_{L^{2}}\|f\|_{C^{0}\left([0, T], H^{3}\right)} .
$$

Proof of the $h^{5}$-bound of Proposition 8. Thanks to and integration by parts, we get

$$
\begin{aligned}
S_{0, k}= & \int_{0}^{T} \dot{\mu} \frac{\lambda_{k, \mu}^{\prime}}{i \lambda_{k, \mu}^{3}} w\left\langle A_{\mu} f, \varphi_{k, \mu}\right\rangle \mathrm{e}^{i \int_{0}^{t} \lambda_{k, \mu}} \mathrm{d} t \\
& -\int_{0}^{T} \dot{w} \frac{1}{i \lambda_{k, \mu}}\left\langle f, \varphi_{k, \mu}\right\rangle \mathrm{e}^{i \int_{0}^{t} \lambda_{k, \mu}} \mathrm{d} t \\
& -\int_{0}^{T} w \frac{1}{i \lambda_{k, \mu}}\left\langle\dot{f}, \varphi_{k, \mu}\right\rangle \mathrm{e}^{i \int_{0}^{t} \lambda_{k, \mu}} \mathrm{d} t \\
& \left.-\int_{0}^{T} \dot{\mu} w \frac{1}{i \lambda_{k, \mu}}\left\langle f, \frac{\mathrm{d} \varphi_{k, \gamma}}{\mathrm{d} \gamma}\right]_{\mu}\right\rangle \mathrm{e}^{i \int_{0}^{t} \lambda_{k, \mu}} \mathrm{d} t .
\end{aligned}
$$

Let us call this decomposition

$$
S_{0}=S_{0, a^{\prime}}+S_{0, b^{\prime}}+S_{0, c^{\prime}}+S_{0, d^{\prime}}
$$

Using the following bound

$$
\left|\frac{\lambda_{k, \mu}^{\prime}}{\lambda_{k, \mu}^{3}}\right| \leqslant \frac{C}{k^{6}}
$$

which is a consequence of (A.1) and (A.11), we get

$$
\left\|S_{0, a^{\prime}}\right\|_{h^{5}} \leqslant C\|\dot{\mu} w\|_{L^{2}}\left\|A_{\mu} f\right\|_{C^{0}\left([0, T], L^{2}\right)} \leqslant C\|\mu\|_{H^{1}}\|w\|_{H^{1}}\|f\|_{C^{0}\left([0, T], H^{2}\right)} .
$$

Using Lemma 1, we get

$$
\left\|S_{0, d^{\prime}}\right\|_{h^{5}} \leqslant C\|\dot{\mu} w\|_{L^{2}}\|f\|_{C^{0}\left([0, T], H^{2}\right)} \leqslant C\|\mu\|_{H^{1}}\|w\|_{H^{1}}\|f\|_{C^{0}\left([0, T], H^{2}\right)} .
$$

We have

$$
S_{0, b^{\prime}}=i \int_{0}^{T} \dot{w}\left(\frac{1}{\lambda_{k, \mu}}-\frac{1}{\lambda_{k}}\right) \frac{1}{\lambda_{k, \mu}}\left\langle A_{\mu} f, \varphi_{k, \mu}\right\rangle \mathrm{e}^{i \int_{0}^{t} \lambda_{k, \mu}} \mathrm{d} t+\frac{i}{\lambda_{k}} \int_{0}^{T} \dot{w}\left\langle f, \varphi_{k, \mu}\right\rangle \mathrm{e}^{i \int_{0}^{t} \lambda_{k, \mu}} \mathrm{d} t .
$$

Thanks to (A.4), the $h^{5}$-norm of the first term in this sum is bounded by $C\|\mu\|_{H^{1}}\|w\|_{H^{1}}\|f\|_{C^{0}\left([0, T], H^{2}\right)}$. The decomposition of $S_{0}$ in $h^{3}$ gives

$$
S_{0, b^{\prime}}=\left(\frac{-1}{i \lambda_{k}} \int_{0}^{T} \dot{w}\left\langle f, \varphi_{k}\right\rangle \mathrm{e}^{i \lambda_{k} t} \mathrm{~d} t\right)_{k \in \mathbb{N}^{*}}+\text { terms with an } h^{5} \text { norm } \leqslant C\|\mu\|_{H^{1}}\|w\|_{H^{1}}\|f\|_{C^{0}\left([0, T], H^{3}\right)} .
$$

Doing the same thing on $S_{0, c^{\prime}}$, we get

$$
S_{0, c^{\prime}}=\left(\frac{-1}{i \lambda_{k}} \int_{0}^{T} w\left\langle\dot{f}, \varphi_{k, \mu}\right\rangle \mathrm{e}^{i \int_{0}^{t} \lambda_{k, \mu} \mathrm{d} t}\right)_{k \in \mathbb{N}^{*}}+\text { terms with an } h^{5} \text { norm } \leqslant C\|\mu\|_{H^{1}}\|w\|_{L^{2}}\|f\|_{C^{1}\left([0, T], H^{3}\right)} .
$$


Proof of the $h^{7}$-bound of Proposition 8. First, one notice that $S_{0, a^{\prime}}$ and $S_{0, d^{\prime}}$ belong to $h^{7}$. Indeed,

$$
S_{0, a^{\prime}}=\frac{\lambda_{k}^{\prime}}{\lambda_{k}^{2}} \int_{0}^{T} \dot{\mu} w\left\langle f, \varphi_{k, \mu}\right\rangle \mathrm{e}^{i \int_{0}^{t} \lambda_{k, \mu}} \mathrm{d} t+\int_{0}^{T}\left(\frac{\lambda_{k, \mu}^{\prime}}{\lambda_{k, \mu}^{2}}-\frac{\lambda_{k}^{\prime}}{\lambda_{k}^{2}}\right) \frac{1}{\lambda_{k, \mu}} \dot{\mu} w\left\langle A_{\mu} f, \varphi_{k, \mu}\right\rangle \mathrm{e}^{i \int_{0}^{t} \lambda_{k, \mu}} \mathrm{d} t .
$$

Using the Cauchy Schwarz inequality in $L^{2}((0, T), \mathbb{C})$, the orthonormality of the family $\left(\varphi_{k, \mu}\right)$ and the inequality

$$
\left|\frac{\lambda_{k, \mu}^{\prime}}{\lambda_{k, \mu}^{2}}-\frac{\lambda_{k}^{\prime}}{\lambda_{k}^{2}}\right| \leqslant \frac{C\|\mu\|_{H^{1}}}{k^{5}}
$$

which is a consequence of (A.3) and (A.11), we get the following bound for $h^{7}$-norm of the second term in $S_{0, a^{\prime}}$

$$
C\|\mu\|_{H^{1}}\|\dot{\mu} w\|_{L^{2}}\left\|A_{\mu} f\right\|_{C^{0}\left([0, T], H^{2}\right)} \leqslant C\|\mu\|_{H^{1}}\|w\|_{H^{1}}\|f\|_{C^{0}\left([0, T], H^{2}\right)} .
$$

Thanks to (A.1) and the $h^{3}$-bound on $S_{0}$, we get the following bound for the $h^{7}$-norm of the first term in $S_{0, a^{\prime}}$

$$
C\|\dot{\mu} w\|_{L^{2}}\|f\|_{C^{0}\left([0, T], H^{3}\right)} \leqslant C\|\mu\|_{H^{1}}\|w\|_{H^{1}}\|f\|_{C^{0}\left([0, T], H^{3}\right)} .
$$

We also have

$$
\left.\left.S_{0, d^{\prime}}=\frac{-1}{i \lambda_{k}} \int_{0}^{T} \dot{\mu} w\left\langle f, \frac{\mathrm{d} \varphi_{k, \gamma}}{\mathrm{d} \gamma}\right]_{\mu(t)}\right\rangle \mathrm{e}^{i \int_{0}^{t} \lambda_{k, \mu}} \mathrm{d} t+\int_{0}^{T}\left(\frac{1}{i \lambda_{k}}-\frac{1}{i \lambda_{k, \mu}}\right) \dot{\mu} w\left\langle f, \frac{\mathrm{d} \varphi_{k, \gamma}}{\mathrm{d} \gamma}\right]_{\mu(t)}\right\rangle \mathrm{e}^{i \int_{0}^{t} \lambda_{k, \mu}} \mathrm{d} t .
$$

Thanks to (A.4) and Lemma 1, we get the following bound for the $h^{7}$-bound of the second term in $S_{0, d^{\prime}}$

$$
C\|\mu\|_{H^{1}}\|\dot{\mu} w\|_{L^{2}}\|f\|_{C^{0}\left([0, T], H^{2}\right)} \leqslant C\|\mu\|_{H^{1}}\|w\|_{H^{1}}\|f\|_{C^{0}\left([0, T], H^{2}\right)} .
$$

For the first term, applying the bound we know on the $h^{5}$-norm of $S_{1}$, we get the following bound

$C\left[\|\dot{\mu} w\|_{L^{2}}\|f\|_{C^{1}\left([0, T], H^{2}\right)}+\|\dot{\mu} w\|_{H^{1}}\|f\|_{C^{0}\left([0, T], H^{2}\right)}\right]$

$$
\leqslant C\left[\|\mu\|_{H^{1}}\|w\|_{H^{1}}\|f\|_{C^{1}\left([0, T], H^{2}\right)}+\|\mu\|_{H^{2}}\|w\|_{H^{1}}\|f\|_{C^{0}\left([0, T], H^{2}\right)}\right] .
$$

Now, we have to study $S_{0, b^{\prime}}$ and $S_{0, c^{\prime}}$ in $h^{7}$. Using (A.12), we get

$$
\frac{1}{\lambda_{k}}-\frac{1}{\lambda_{k, \mu}}=\frac{\mu}{3 \lambda_{k}^{2}}+d_{k}(\mu) \text { where }\left|d_{k}(\mu)\right| \leqslant \frac{C\|\mu\|_{H^{1}}}{k^{6}} .
$$

Thus,

$$
\begin{aligned}
S_{0, b^{\prime}}=\frac{-1}{i \lambda_{k}} \int_{0}^{T} \dot{w}\left\langle f, \varphi_{k, \mu}\right\rangle \mathrm{e}^{i \int_{0}^{t} \lambda_{k, \mu}} \mathrm{d} t+\frac{1}{3 \lambda_{k}^{2}} \int_{0}^{T} \mu \dot{w}\left\langle f, \varphi_{k, \mu}\right\rangle \mathrm{e}^{i \int_{0}^{t} \lambda_{k, \mu}} \mathrm{d} t & \\
& +\int_{0}^{T} \mathrm{~d}_{k}(\mu) \dot{w} \frac{1}{\lambda_{k, \mu}}\left\langle A_{\mu} f, \varphi_{k, \mu}\right\rangle \mathrm{e}^{i \int_{0}^{t} \lambda_{k, \mu}} \mathrm{d} t .
\end{aligned}
$$

Let us call this decomposition

Thanks to (4.7), we get

$$
S_{0, b^{\prime}}=S_{0, b^{\prime}, 1}+S_{0, b^{\prime}, 2}+S_{0, b^{\prime}, 3}
$$

$$
\left\|S_{0, b^{\prime}, 3}\right\|_{h^{7}} \leqslant C\|\mu\|_{H^{1}}\|w\|_{H^{1}}\|f\|_{C^{0}\left([0, T], H^{2}\right)} .
$$


Using the bound on the $h^{3}$-norm of $S_{0}$, we get

$$
\left\|S_{0, b^{\prime}, 2}\right\|_{h^{7}} \leqslant C\|\mu \dot{w}\|_{L^{2}}\|f\|_{C^{0}\left([0, T], H^{3}\right)} \leqslant C\|\mu\|_{H^{1}}\|w\|_{H^{1}}\|f\|_{C^{0}\left([0, T], H^{3}\right)} .
$$

Thanks to the decomposition of $S_{0}$ in $h^{5}$, we get

$$
\begin{aligned}
S_{0, b^{\prime}}= & \frac{-1}{\lambda_{k}^{2}} \int_{0}^{T}\left(\ddot{w}\left\langle f, \varphi_{k}\right\rangle+\dot{w}\left\langle\dot{f}, \varphi_{k}\right\rangle\right) \mathrm{e}^{i \lambda_{k} t} \mathrm{~d} t \\
& + \text { terms with an } h^{7} \text { norm } \leqslant C\|\mu\|_{H^{1}}\left[\|w\|_{H^{2}}\|f\|_{C^{0}\left([0, T], H^{3}\right)}+\|w\|_{H^{1}}\|f\|_{C^{1}\left([0, T], H^{3}\right)}\right] .
\end{aligned}
$$

Working in the same way on $S_{0, c^{\prime}}$, we get

$$
\begin{aligned}
S_{0, c^{\prime}}= & \frac{-1}{\lambda_{k}^{2}} \int_{0}^{T}\left(\dot{w}\left\langle\dot{f}, \varphi_{k}\right\rangle+w\left\langle\ddot{f}, \varphi_{k}\right\rangle\right) \mathrm{e}^{i \lambda_{k} t} \mathrm{~d} t \\
& + \text { terms with an } h^{7} \text { norm } \leqslant C\|\mu\|_{H^{1}}\left[\|w\|_{H^{1}}\|f\|_{C^{1}\left([0, T], H^{3}\right)}+\|w\|_{L^{2}}\|f\|_{C^{2}\left([0, T], H^{3}\right)}\right]
\end{aligned}
$$

4.4.2. Study of $M_{\left(\psi_{0}, u\right)}-M_{\left(\psi^{\text {ref }}(0), 0\right)}$

In order to prove Proposition 7, we cut $\left[M_{\left(\psi_{0}, u\right)}-M_{\left(\psi^{r e f}(0), 0\right)}\right](v)$ in several pieces on which we prove the bounds of Proposition 7 one by one. We introduce the sequences $\mathrm{d} M_{j}(v):=\left(\mathrm{d} M_{j}(v)_{k}\right)_{k \in \mathbb{N}^{*}}$, for $j=1,2,3,4,5$,

where

$$
\begin{aligned}
& \mathrm{d} M_{1}(v)_{1}:=\int_{0}^{T} \dot{v}\left(\left\langle x^{2} \psi, \psi\right\rangle-\left\langle x^{2} \psi_{r e f}, \psi_{r e f}\right\rangle\right) \mathrm{d} t, \\
& \mathrm{~d} M_{1}(v)_{k}:=\int_{0}^{T} \dot{v}\left(\left\langle x^{2} \psi, \varphi_{k, \mu}\right\rangle \mathrm{e}^{i \int_{0}^{t} \lambda_{k, \mu(s)} \mathrm{d} s}-\left\langle x^{2} \psi_{r e f}, \varphi_{k}\right\rangle \mathrm{e}^{i \lambda_{k} t}\right) \mathrm{d} t, \text { for every } k \geqslant 2, \\
& \mathrm{~d} M_{2}(v)_{1}:=\int_{0}^{T} u v\left(\left\langle x^{2} \psi, \psi\right\rangle-\left\langle x^{2} \psi_{r e f}, \psi_{r e f}\right\rangle\right) \mathrm{d} t, \\
& \mathrm{~d} M_{2}(v)_{k}:=\int_{0}^{T} u v\left(\left\langle x^{2} \psi, \varphi_{k, \mu}\right\rangle \mathrm{e}^{i \int_{0}^{t} \lambda_{k, \mu(s)} \mathrm{d} s}-\left\langle x^{2} \psi_{r e f}, \varphi_{k}\right\rangle \mathrm{e}^{i \lambda_{k} t}\right) \mathrm{d} t, \text { for every } k \geqslant 2, \\
& \mathrm{~d} M_{3}(v)_{1}:=0, \\
& \mathrm{~d} M_{3}(v)_{k}:=\int_{0}^{T}\left(\dot{\alpha}\left\langle\psi, \varphi_{k, \mu}\right\rangle \mathrm{e}^{i \int_{0}^{t} \lambda_{k, \mu(s)} \mathrm{d} s}-\dot{\alpha}_{0}\left\langle\psi_{r e f}, \varphi_{k}\right\rangle \mathrm{e}^{i \lambda_{k} t}\right) \mathrm{d} t, \forall k \geqslant 2
\end{aligned}
$$

$$
\alpha_{0}:=\frac{\left\langle\Psi_{0}, \varphi_{1}\right\rangle}{\left\langle\psi_{\text {ref }}, \varphi_{1}\right\rangle} \text { and }\left\{\begin{array}{l}
i \dot{\Psi}_{0}=-\Psi_{0}^{\prime \prime}+\dot{v} x^{2} \psi_{r e f} \\
\Psi_{0}(t, 0)=\Psi_{0}(t, 1)=0 \\
\Psi_{0}(0)=0
\end{array}\right.
$$$$
\mathrm{d} M_{4}(v)_{1}:=0
$$$$
\left.\mathrm{d} M_{4}(v)_{k}:=\int_{0}^{T} \dot{\mu}\left\langle\Psi, \frac{\mathrm{d} \varphi_{k, \gamma}}{\mathrm{d} \gamma}\right]_{\mu}\right\rangle \mathrm{e}^{i \int_{0}^{t} \lambda_{k, \mu(s)} \mathrm{d} s} \mathrm{~d} t, \forall k \geqslant 2,
$$$$
\mathrm{d} M_{5}(v)_{1}:=0
$$$$
\left.\mathrm{d} M_{5}(v)_{k}:=\int_{0}^{T} \dot{\mu} \alpha\left\langle\psi, \frac{\varphi_{k, \gamma}}{\mathrm{d} \gamma}\right]_{\mu}\right\rangle \mathrm{e}^{i \int_{0}^{t} \lambda_{k, \mu(s)} \mathrm{d} s} \mathrm{~d} t, \forall k \geqslant 2 .
$$

First, let us remark that, when $\delta_{4} \leqslant 1$ then (see App. B)

$$
\begin{aligned}
& \|\psi\|_{C^{0}\left([0, T], H^{2}\right)} \leqslant C \\
& \|\dot{\psi}\|_{C^{0}\left([0, T], H^{2}\right)},\|\psi\|_{C^{0}\left([0, T], H^{4}\right)} \leqslant C \\
& \|\ddot{\psi}\|_{C^{0}\left([0, T], H^{2}\right)},\|\dot{\psi}\|_{C^{0}\left([0, T], H^{4}\right)},\|\psi\|_{C^{0}\left([0, T], H^{6}\right)} \leqslant C\left(1+\delta_{6}\right) .
\end{aligned}
$$


Study of $\mathbf{d} M_{1}$. We have

$$
\left|\mathrm{d} M_{1}(v)_{1}\right| \leqslant C\|v\|_{H^{1}}\left\|\psi-\psi_{r e f}\right\|_{C^{0}\left([0, T], L^{2}\right)} \leqslant \delta_{4}\|v\|_{H^{1}}
$$

Thanks to Proposition 8, we have, for $k \geqslant 2$,

$$
\begin{aligned}
\mathrm{d} M_{1}(v)_{k}= & \frac{1}{\lambda_{k}} \int_{0}^{T} \dot{v}\left\langle A\left[x^{2}\left(\psi-\psi_{r e f}\right)\right], \varphi_{k}\right\rangle \mathrm{e}^{i \lambda_{k} t} \mathrm{~d} t \\
& + \text { terms with an } h^{3} \text { norm bounded by } \\
& C\|\mu\|_{H^{1}}\|v\|_{H^{1}}\|\psi\|_{C^{0}\left([0, T], H^{3}\right)} \leqslant C \delta_{4}\|v\|_{H^{1}}
\end{aligned}
$$

Applying again Proposition 8, we get

$$
\left\|\mathrm{d} M_{1}\right\|_{h^{3}} \leqslant C\left[\|v\|_{H^{1}}\left\|\psi-\psi_{r e f}\right\|_{C^{0}\left([0, T], H^{3}\right)}+\delta_{4}\|v\|_{H^{1}} \leqslant C \delta_{4}\|v\|_{H^{1}} .\right.
$$

Thanks to Proposition 8 , we have, for $k \geqslant 2$,

$$
\begin{aligned}
\mathrm{d} M_{1}(v)_{k}= & \frac{-1}{i \lambda_{k}} \int_{0}^{T}\left(\ddot{v}\left\langle x^{2}\left(\psi-\psi_{r e f}\right), \varphi_{k}\right\rangle+\dot{v}\left\langle x^{2}\left(\dot{\psi}-\dot{\psi}_{r e f}\right), \varphi_{k}\right\rangle\right) \mathrm{e}^{i \lambda_{k} t} \mathrm{~d} t \\
& + \text { terms with an } h^{5} \text { norm bounded by } \\
& C\|\mu\|_{H^{1}}\left[\|v\|_{H^{2}}\|\psi\|_{C^{0}\left([0, T], H^{3}\right)}+\|v\|_{H^{1}}\|\psi\|_{C^{1}\left([0, T], H^{3}\right)}\right] \leqslant C\left[\delta_{4}\|v\|_{H^{2}}+\delta_{6}\|v\|_{H^{1}}\right] .
\end{aligned}
$$

Applying again Proposition 8, we get

$$
\begin{aligned}
\left\|\mathrm{d} M_{1}\right\|_{h^{5}} & \leqslant C\left[\|\ddot{v}\|_{L^{2}}\left\|\psi-\psi_{r e f}\right\|_{C^{0}\left([0, T], H^{3}\right)}+\|\dot{v}\|_{L^{2}}\left\|\dot{\psi}-\dot{\psi}_{r e f}\right\|_{C^{0}\left([0, T], H^{3}\right.}+\delta_{4}\|v\|_{H^{2}}+\delta_{6}\|v\|_{H^{1}}\right] \\
& \leqslant C\left[\delta_{4}\|v\|_{H^{2}}+\delta_{6}\|v\|_{H^{1}}\right] .
\end{aligned}
$$

We study $\mathrm{d} M_{1}$ in $h^{7}$ in the same way.

Study of $\mathbf{d} M_{2}$. For the study of $\mathrm{d} M_{2}$, we apply Proposition 8 . We have

$$
\begin{aligned}
\left\|\mathrm{d} M_{2}\right\|_{h^{3}} \leqslant & \leqslant\|u v\|_{L^{2}}\left(1+\left\|\psi-\psi_{r e f}\right\|_{C^{0}\left([0, T], H^{3}\right)}\right) \\
\left\|\mathrm{d} M_{2}\right\|_{h^{5}} \leqslant & \leqslant C\left[\|u v\|_{H^{1}} \delta_{4}\right. \\
\| & C\left[\|v\|_{H^{2}}\left(1+\left\|\psi-\delta_{4}+\right\| v \|_{H^{1}} \delta_{6}\right]\right. \\
\left\|\mathrm{d} M_{2}\right\|_{h^{7}} \leqslant & C\left[\|u v\|_{H^{2}}\left(1+\left\|\psi-\psi_{r e f}\right\|_{C^{0}\left([0, T], H^{3}\right)}\right)+\|u v\|_{H^{1}}\left(1+\left\|\psi-\psi_{r e f}\right\|_{C^{0}\left([0, T], H^{5}\right)}\right)+\|u v\|_{L^{2}}\left(1+\left\|\psi-\psi_{r e f}\right\|_{C^{0}\left([0, T], H^{5}\right)}\right)\right] \\
& +\|u v\|_{L^{2}}\left(1+\left\|\psi-\psi_{r e f}\right\|_{C^{0}\left([0, T], H^{7}\right)}\right)+\|\mu\|_{H^{2}}\|u v\|_{H^{1}}\left(1+\left\|\psi-\psi_{r e f}\right\|_{C^{0}\left([0, T], H^{2}\right)}\right] \\
\leqslant & C\left[\|v\|_{H^{3} \delta_{4}}+\|v\|_{H^{2}} \delta_{6}+\|v\|_{H^{1}} \delta_{8}\right] .
\end{aligned}
$$

Study of $\mathbf{d} M_{3}$. We detail the study of $\mathrm{d} M_{3}$ in $h^{3}$, the study in $h^{5}$ and $h^{7}$ can be done in the same way. Using the same arguments as for $S_{0}$ in $h^{3}$, and the bound

$$
\|\dot{\alpha}\|_{L^{2}} \leqslant C\|v\|_{H^{1}}
$$

we get

$$
\begin{aligned}
\mathrm{d} M_{3}(v)= & \int_{0}^{T}\left(\dot{\alpha}-\dot{\alpha}_{0}\right)\left\langle x^{2} \psi, \varphi_{k}\right\rangle \mathrm{e}^{i \lambda_{k} t} \mathrm{~d} t+\int_{0}^{T} \dot{\alpha}_{0}\left\langle x^{2}\left(\psi-\psi_{r e f}\right), \varphi_{k}\right\rangle \mathrm{e}^{i \lambda_{k} t} \mathrm{~d} t \\
& + \text { terms with an } h^{3} \text { norm bounded by } C \delta_{4}\|v\|_{H^{1}}
\end{aligned}
$$


One can prove that

$$
\left\|\dot{\alpha}-\dot{\alpha}_{0}\right\|_{L^{2}} \leqslant C \delta_{4}\|v\|_{H^{1}}
$$

which, with Proposition 8, gives the conclusion.

Study of $\mathbf{d} M_{4}$. Thanks to Proposition 9, we have

$$
\begin{aligned}
\left\|\mathrm{d} M_{4}(v)\right\|_{h^{3}} \leqslant & C\|\mu\|_{H^{1}}\|\Psi\|_{C^{0}\left([0, T], H^{2}\right)} \\
\left\|\mathrm{d} M_{4}(v)\right\|_{h^{5}} \leqslant & C\left[\|\mu\|_{H^{2}}\|\Psi\|_{C^{0}\left([0, T], H^{2}\right)}+\|\mu\|_{H^{1}}\|\Psi\|_{C^{1}\left([0, T], H^{2}\right)}\right] \\
\left\|\mathrm{d} M_{4}(v)\right\|_{h^{7}} \leqslant & C\left[\|\mu\|_{H^{3}}\|\Psi\|_{C^{0}\left([0, T], H^{2}\right)}+\|\mu\|_{H^{2}}\|\Psi\|_{C^{1}\left([0, T], H^{2}\right)}+\|\mu\|_{H^{1}}\|\Psi\|_{C^{2}\left([0, T], H^{2}\right)}\right. \\
& \left.+\|\mu\|_{H^{2}}\|\mu\|_{H^{1}}\|\Psi\|_{C^{0}\left([0, T], H^{2}\right)}\right] .
\end{aligned}
$$

Using Appendix B, we get, when $\delta_{4} \leqslant 1$,

$$
\begin{aligned}
& \|\Psi\|_{C^{0}\left([0, T], H^{2}\right)} \leqslant C\|v\|_{H^{1}} \\
& \|\Psi\|_{C^{1}\left([0, T], H^{2}\right)} \leqslant C\|v\|_{H^{2}}, \\
& \|\Psi\|_{C^{2}\left([0, T], H^{2}\right)} \leqslant C\left[\|v\|_{H^{3}}+\delta_{6}\|v\|_{H^{1}}\right]
\end{aligned}
$$

which gives the conclusion.

Study of $\mathbf{d} M_{5}$. Thanks to Proposition 9, we have

$$
\begin{aligned}
& \left\|\mathrm{d} M_{5}(v)\right\|_{h^{3}} \leqslant C\|\dot{\mu} \alpha\|_{L^{2}}\|\psi\|_{C^{0}\left([0, T], H^{2}\right)}, \\
& \left\|\mathrm{d} M_{5}(v)\right\|_{h^{5}} \leqslant C\left[\|\dot{\mu} \alpha\|_{H^{1}}\|\psi\|_{C^{0}\left([0, T], H^{2}\right)}+\|\dot{\mu} \alpha\|_{L^{2}}\|\psi\|_{C^{1}\left([0, T], H^{2}\right)}\right], \\
& \left\|\mathrm{d} M_{5}(v)\right\|_{h^{7}} \leqslant C\left[\|\dot{\mu} \alpha\|_{H^{2}}\|\psi\|_{C^{0}\left([0, T], H^{2}\right)}+\|\dot{\mu} \alpha\|_{H^{1}}\|\psi\|_{C^{1}\left([0, T], H^{2}\right)}+\|\dot{\mu} \alpha\|_{L^{2}}\|\psi\|_{C^{2}\left([0, T], H^{2}\right)}\right] .
\end{aligned}
$$

Thanks to Appendix B, we have

$$
\|\alpha\|_{H^{1}} \leqslant C\|v\|_{H^{1}} \quad \text { and } \quad\|\alpha\|_{H^{1}} \leqslant C\|v\|_{H^{2}} .
$$

Thus

$$
\|\dot{\mu} \alpha\|_{L^{2}} \leqslant C \delta_{4}\|v\|_{H^{1}}, \quad\|\dot{\mu} \alpha\|_{H^{1}} \leqslant C \delta_{6}\|v\|_{H^{1}}, \quad\|\dot{\mu} \alpha\|_{H^{2}} \leqslant C\left[\delta_{8}\|v\|_{H^{1}}+\delta_{6}\|v\|_{H^{2}}\right],
$$

which gives the conclusion.

\section{LOCAL CONTROLLABILITY AROUND $\boldsymbol{\psi}_{\mathbf{1}}$}

\subsection{Local controllability up to codimension one around $\psi_{1}$}

Let us introduce the following closed subspace of $L^{2}((0,1), \mathbb{C})$,

$$
V:=\operatorname{Span}\left\{\varphi_{k} ; k \geqslant 2\right\}
$$

and the orthogonal projection $\mathcal{P}: L^{2}((0,1), \mathbb{C}) \rightarrow V$. We admit the following result, that will be proved in Section 6 .

Theorem 9. Let $T:=2 / \pi$ and $\epsilon>0$. There exists $C>0, \delta>0$ and a continuous map

$$
\begin{array}{rllllc}
\Gamma: & \mathcal{N} & \times & \widetilde{\mathcal{N}} & \rightarrow & \overline{H_{0}^{2}}((0, T), \mathbb{R}) \\
\left(\psi_{0}\right. & , & \left.\widetilde{\psi_{f}}\right) & \mapsto & u
\end{array}
$$


where

$$
\begin{gathered}
\mathcal{N}:=\left\{\psi \in \mathcal{S} \cap H_{(0)}^{5+\epsilon}((0,1), \mathbb{C}) ;\left\|\psi-\varphi_{1}\right\|_{H^{5+\epsilon}}\langle\delta\},\right. \\
\widetilde{\mathcal{N}}:=\left\{\widetilde{\psi} \in V \cap H_{(0)}^{5+\epsilon}((0,1), \mathbb{C}) ;\|\widetilde{\psi}\|_{L^{2}} \leqslant 1 \text { and }\|\widetilde{\psi}\|_{H^{5+\epsilon}}\langle\delta\},\right.
\end{gathered}
$$

such that, for every $\left(\psi_{0}, \widetilde{\psi_{f}}\right) \in \mathcal{N} \times \widetilde{\mathcal{N}}$

$$
\left\|\Gamma\left(\psi_{0}, \widetilde{\psi_{f}}\right)\right\|_{H_{0}^{2}} \leqslant C\left[\left\|\mathcal{P} \psi_{0}\right\|_{H^{5+\epsilon}}+\left\|\widetilde{\psi_{f}}\right\|_{H^{5+\epsilon}}\right]
$$

and the trajectory of $(\Sigma)$ with $\psi(0)=\psi_{0}$ and control $u=\Gamma\left(\psi_{0}, \widetilde{\psi_{f}}\right)$ satisfies $\mathcal{P} \psi(T)=\widetilde{\psi_{f}}$.

Remark 10. This theorem probably holds with any $T>0$. Indeed, the linearized system around $\left(\psi_{1}, u \equiv 0\right)$ is controllable in any positive time (see Th. 4) and the application of the Nash-Moser theorem does not introduce a positive minimal time for the controllability that is not needed for the linearized system (see Rem. 9). The only point which misses to get theorem with any $T>0$ is the controllability of the linearized system around $\left(\psi_{1}, u \equiv 0\right)$ WITH the bounds (3.8) and (3.9) (corresponding to $\left.v=0\right)$ in any time $T>0$. Theses bounds are easier to prove with $T=2 / \pi$.

\subsection{Second order term}

The goal of this section is the proof of the following result.

Proposition 10. Let $T:=2 / \pi$. There exist $v_{ \pm} \in \overline{H^{4}} \cap H_{0}^{3}((0, T), \mathbb{R})$ and $\nu_{ \pm} \in \overline{H_{0}^{3}}((0, T), \mathbb{R})$ such that the solutions of the following systems

$$
\left\{\begin{array} { l } 
{ i \dot { \Psi } _ { \pm } = - \Psi _ { \pm } ^ { \prime \prime } + \dot { v } _ { \pm } ( t ) x ^ { 2 } \psi _ { 1 } ( t ) , } \\
{ \Psi _ { \pm } ( t , 0 ) = \Psi _ { \pm } ( t , 1 ) = 0 , } \\
{ \Psi _ { \pm } ( 0 ) = 0 , }
\end{array} \quad \left\{\begin{array}{l}
i \dot{\xi}_{ \pm}=-\xi_{ \pm}^{\prime \prime}+\dot{v}_{ \pm}(t) x^{2} \Psi_{ \pm}+\left[\dot{\nu}_{ \pm}-4 v_{ \pm}^{2}\right](t) x^{2} \psi_{1}(t) \\
\xi_{ \pm}(t, 0)=\xi_{ \pm}(t, 1)=0 \\
\xi_{ \pm}(0)=0
\end{array}\right.\right.
$$

satisfy $\Psi_{ \pm}(T)=0$ and $\xi_{ \pm}(T)= \pm i \psi_{1}(T)$.

Let us introduce the following subspace of $L^{2}((0,2 / \pi), \mathbb{C})$

$$
\mathcal{X}:=\operatorname{Span}\left\{\mathrm{e}^{i\left(\lambda_{k}-\lambda_{1}\right) t} ; k \in \mathbb{N}^{*}\right\} .
$$

We denote by $\mathcal{X}^{\perp}$ the orthogonal subspace to $\mathcal{X}$ in $L^{2}((0,2 / \pi), \mathbb{C})$.

Proposition 11. Let $T:=2 / \pi$. There exists $v \in \bar{H}^{4} \cap H_{0}^{3}((0, T), \mathbb{R})$ such that $\dot{v} \in \mathcal{X}^{\perp}$ and

$$
\int_{0}^{T} \dot{v}(t)\left\langle x^{2} \Psi(t), \varphi_{1}\right\rangle \mathrm{e}^{i \lambda_{1} t} \mathrm{~d} t-4\left\langle x^{2} \varphi_{1}, \varphi_{1}\right\rangle \int_{0}^{T} v(t)^{2} \mathrm{~d} t \in(0,+\infty)(\text { resp. } \in(-\infty, 0))
$$

where $\Psi$ is the solution of

$$
\left\{\begin{array}{l}
i \dot{\Psi}=-\Psi^{\prime \prime}+\dot{v}(t) x^{2} \psi_{1}(t) \\
\Psi(t, 0)=\Psi(t, 1)=0 \\
\Psi(0)=0
\end{array}\right.
$$

Remark 11. When $\dot{v} \in \mathcal{X}^{\perp}$ the left hand side of (5.1) belongs to $\mathbb{R}$. Indeed, we have

$$
\Psi(t)=\sum_{k=1}^{\infty} y_{k}(t) \varphi_{k} \text { where } y_{k}(t)=-i\left\langle x^{2} \varphi_{1}, \varphi_{k}\right\rangle \int_{0}^{t} \dot{v}(\tau) \mathrm{e}^{i\left(\lambda_{k}-\lambda_{1}\right) \tau} \mathrm{d} \tau \mathrm{e}^{-i \lambda_{k} t},
$$


thus

$$
\int_{0}^{T} \dot{v}(t)\left\langle x^{2} \Psi(t), \varphi_{1}\right\rangle \mathrm{e}^{i \lambda_{1} t} \mathrm{~d} t=-i \sum_{k=1}^{\infty}\left\langle x^{2} \varphi_{1}, \varphi_{k}\right\rangle^{2} f_{k}(v)
$$

where

$$
f_{k}(v):=\int_{0}^{T} \dot{v}(t) \mathrm{e}^{i\left(\lambda_{k}-\lambda_{1}\right) t} \int_{0}^{t} \dot{v}(\tau) \mathrm{e}^{i\left(\lambda_{1}-\lambda_{k}\right) \tau} \mathrm{d} \tau \mathrm{d} t .
$$

Thanks to an integration by parts, the assumption $\dot{v} \in \mathcal{X}^{\perp}$ gives, for every $k \in \mathbb{N}^{*}, f_{k}(v) \in i \mathbb{R}$.

Proof. We consider the candidate

$$
\dot{v}(t):=\cos \left(n_{1} \pi^{2} t\right)-\cos \left(n_{2} \pi^{2} t\right)
$$

where $n_{1}, n_{2} \in \mathbb{N}^{*}, n_{1} \neq n_{2}$ and $n_{1}, n_{2} \notin\left\{k^{2}-1 ; k \in \mathbb{N}^{*}\right\}$. Then $\dot{v} \in \mathcal{X}^{\perp}$, and $v(t):=\int_{0}^{t} \dot{v}(\tau) \mathrm{d} \tau$ belongs to $\overline{H^{4}} \cap H_{0}^{3}((0, T), \mathbb{R})$. Explicit computations give $f_{1}(v)=0$ and, for $k \geqslant 2$,

$$
f_{k}(v)=\frac{1}{2 i \pi^{3}}\left(\frac{1}{-n_{1}+k^{2}-1}+\frac{1}{n_{1}+k^{2}-1}+\frac{1}{-n_{2}+k^{2}-1}+\frac{1}{n_{2}+k^{2}-1}\right) .
$$

Thus,

$$
\int_{0}^{T} \dot{v}(t)\left\langle x^{2} \Psi(t), \varphi_{1}\right\rangle \mathrm{e}^{i \lambda_{1} t} \mathrm{~d} t-4\left\langle x^{2} \varphi_{1}, \varphi_{1}\right\rangle \int_{0}^{T} v(t)^{2} \mathrm{~d} t=A\left(n_{1}\right)+A\left(n_{2}\right)
$$

where

$$
A(n):=-\frac{4}{n^{2} \pi^{5}}\left(\frac{1}{3}-\frac{1}{2 \pi^{2}}\right)-\frac{64}{\pi^{7}} \sum_{k=2}^{\infty} \frac{k^{2}}{(k+1)^{3}(k-1)^{3}\left(n+k^{2}-1\right)\left(-n+k^{2}-1\right)} .
$$

With $\left(n_{1}, n_{2}\right)=(1,2)$, we get $A\left(n_{1}\right), A\left(n_{2}\right)<0$ and with $\left(n_{1}, n_{2}\right)=(4,5)$, we get $A\left(n_{1}\right), A\left(n_{2}\right)>0$.

Proof of Proposition 10. Let $v \in \overline{H^{4}} \cap H_{0}^{3}((0, T), \mathbb{R})$ be such that

$$
\int_{0}^{T} \dot{v}(t)\left\langle x^{2} \Psi(t), \varphi_{1}\right\rangle \mathrm{e}^{i \lambda_{1} t} \mathrm{~d} t-4\left\langle x^{2} \varphi_{1}, \varphi_{1}\right\rangle \int_{0}^{T} v(t)^{2} \mathrm{~d} t=-1(\text { resp. }+1),
$$

such a $v$ exists thanks to Proposition 11. The assumption $\dot{v} \in \mathcal{X}^{\perp}$ gives $\Psi(T)=0$. For $\nu \in \overline{H_{0}^{1}}((0, T), \mathbb{R})$, we have $\xi(T)=\sum_{k=1}^{\infty} z_{k}(T) \varphi_{k}$ where

$$
z_{k}(T):=-i \int_{0}^{T}\left(\dot{v}(t)\left\langle x^{2} \Psi(t), \varphi_{k}\right\rangle+\left(\dot{\nu}-4 v^{2}\right)(t)\left\langle x^{2} \varphi_{1}, \varphi_{k}\right\rangle \mathrm{e}^{-i \lambda_{1} t}\right) \mathrm{e}^{i \lambda_{k} t} \mathrm{~d} t \mathrm{e}^{-i \lambda_{k} T} .
$$

Thus, the equality $\xi(T)=i \psi_{1}(T)\left(\right.$ resp. $\left.\xi(T)=-i \psi_{1}(T)\right)$ is equivalent to (5.2) and for every $k \geqslant 2$

$$
\int_{0}^{T} \dot{\nu}(t) \mathrm{e}^{i\left(\lambda_{k}-\lambda_{1}\right) t} \mathrm{~d} t=4 \int_{0}^{T} v^{2}(t) \mathrm{e}^{i\left(\lambda_{k}-\lambda_{1}\right) t} \mathrm{~d} t-\frac{1}{\left\langle x^{2} \varphi_{1}, \varphi_{k}\right\rangle} \int_{0}^{T} \dot{v}(t)\left\langle x^{2} \Psi(t), \varphi_{k}\right\rangle \mathrm{e}^{i \lambda_{k} t} \mathrm{~d} t .
$$

There exists $\nu \in \overline{H_{0}^{3}}((0, T), \mathbb{R})$ with these prescribed Fourier coefficients if and only if the right hand side of (5.3) belongs to $h^{4}\left(\mathbb{N}^{*}, \mathbb{C}\right)$. This condition is satisfied when $v \in \overline{H^{4}} \cap H_{0}^{3}((0, T), \mathbb{R})$. 


\subsection{Proof of Theorem 6}

In all this section $T:=2 / \pi, \epsilon \in(0,1)$. Let $\rho \in \mathbb{R}, \psi_{0}, \psi_{f} \in H^{5+\epsilon}((0,1), \mathbb{C})$. Let us consider, for $t \in[0, T]$,

$$
u(t):=\sqrt{|\rho|} v+|\rho| \nu
$$

where $v:=v_{+}, \nu:=\nu_{+}$if $\rho \geqslant 0, v:=v_{-}, \nu:=\nu_{-}$if $\rho \leqslant 0$ and $v_{ \pm}, \nu_{ \pm}$are defined in Proposition 10. Let $\psi$ be the solution of $(\Sigma)$ on $[0, T]$ with control $u$ and such that $\psi(0)=\psi_{0}$. Since $u \in \overline{H_{0}^{3}}((0, T), \mathbb{R})$, we have $\psi(T) \in H^{5+\epsilon}((0,1), \mathbb{C})$.

Proposition 12. There exists a constant $\mathcal{C}$ such that, for every $\rho \in(-1,1)$, we have

$$
\left\|\psi(T)-(1+i \rho) \varphi_{1}\right\|_{H^{5+\epsilon}} \leqslant \mathcal{C}\left[\left\|\psi_{0}-\varphi_{1}\right\|_{H^{5+\epsilon}}+|\rho|^{3 / 2}\right] .
$$

Proof. We have $\psi(T)-(1+i \rho) \varphi_{1}=(\psi-Z)(T)$ where $Z:=\psi_{1}+\Psi+\xi$ and

$$
\left\{\begin{array} { l } 
{ i \dot { \Psi } = - \Psi ^ { \prime \prime } + \sqrt { | \rho | } \dot { v } x ^ { 2 } \psi _ { 1 } , } \\
{ \Psi ( t , 0 ) = \Psi ( t , 1 ) = 0 , } \\
{ \Psi ( 0 ) = 0 , }
\end{array} \quad \left\{\begin{array}{l}
i \dot{\xi}=-\xi^{\prime \prime}+\sqrt{|\rho|} \dot{v} x^{2} \Psi_{1}+|\rho|\left(\dot{\nu}-4 v^{2}\right) x^{2} \psi_{1}, \\
\xi(t, 0)=\xi(t, 1)=0, \\
\xi(0)=0 .
\end{array}\right.\right.
$$

The function $\Delta:=\psi-Z$ solves

$$
\left\{\begin{array}{l}
i \dot{\Delta}=-\Delta^{\prime \prime}+\left[\dot{u}-4 u^{2}\right] \Delta-\sqrt{|\rho|} \dot{v} x^{2} \xi-|\rho| \dot{\nu} x^{2}(\Psi+\xi)+4\left[2|\rho|^{3 / 2} v \nu+|\rho|^{2} \nu^{2}\right] \psi_{1}+4 u^{2}(\Psi+\xi), \\
\Delta(0)=\psi_{0}-\varphi_{1}
\end{array}\right.
$$

We get (5.4) thanks to Propositions 20 and 21 in Appendix B and an interpolation inequality.

Now, we use the local controllability up to codimension one around $\varphi_{1}$. Let $\delta>0$ be as in Theorem 10 . We assume

Then, we have

$$
\begin{aligned}
& \left\|\psi_{0}-\varphi_{1}\right\|_{H^{5+\epsilon}}\left\langle\frac{\delta}{3 \mathcal{C}}, \quad\left\|\psi_{f}-\varphi_{1}\right\|_{H^{5+\epsilon}}\langle\delta\right. \\
& |\rho|\left\langle\eta:=\min \left\{\frac{\delta}{3\left\|\varphi_{1}\right\|_{H^{5+\epsilon}}},\left(\frac{\delta}{3 \mathcal{C}}\right)^{2 / 3}\right\} .\right.
\end{aligned}
$$

$$
\left\|\psi(T)-\varphi_{1}\right\|_{H^{5+\epsilon}}<\delta \text { and }\left\|\mathcal{P} \psi_{f}\right\|_{H^{5+\epsilon}}<\delta
$$

so there exists $u \in H_{0}^{2}((T, 2 T), \mathbb{R})$ such that $\mathcal{P}(\psi(2 T))=\mathcal{P} \psi_{f}$ and

$$
\begin{aligned}
\|u\|_{H_{0}^{2}((T, 2 T), \mathbb{R})} & \leqslant C\left[\left\|\mathcal{P}\left(\psi(T)-\varphi_{1}\right)\right\|_{H^{5+\epsilon}}+\left\|\mathcal{P} \psi_{f}\right\|_{H^{5+\epsilon}}\right] \\
& \leqslant C_{0}\left[\left\|\psi_{0}-\varphi_{1}\right\|_{H^{5+\epsilon}}+|\rho|^{3 / 2}+\left\|\psi_{f}-\varphi_{1}\right\|_{H^{5+\epsilon}}\right] .
\end{aligned}
$$

Moreover, we have

$$
\left\langle\psi(2 T), \varphi_{1}\right\rangle=1+i \rho-i \int_{T}^{2 T}\left[\dot{u}-4 u^{2}\right](t)\left\langle x^{2} \psi(t), \varphi_{1}\right\rangle \mathrm{e}^{i \lambda_{1} t} \mathrm{~d} t
$$

thus

We define the map

$$
\left|\Im\left(\left\langle\psi(2 T), \varphi_{1}\right\rangle\right)-\rho\right| \leqslant C_{1}\|u\|_{H_{0}^{1}((T, 2 T), \mathbb{R})} .
$$

$$
\begin{array}{rlc}
F:(-\eta, \eta) & \rightarrow & \mathbb{R} \\
\rho & \mapsto & \Im\left\langle\psi(2 T), \varphi_{1}\right\rangle
\end{array}
$$


Let $\tau:=\min \left\{\eta, \frac{1}{\left(4 C_{0} C_{1}\right)^{2}}\right\}$ and let us assume also that

$$
\left\|\psi_{0}-\varphi_{1}\right\|_{H^{5+\epsilon}}+\left\|\psi_{f}-\varphi_{1}\right\|_{H^{5+\epsilon}} \leqslant \frac{\tau}{4 C_{0} C_{1}}
$$

then $F(\tau)>\tau / 2>0$ and $F(-\tau)<-\tau / 2<0$ thus, $F$ is surjective on a neighborhood of zero. This ends the Proof of Theorem 6 .

\section{Local Controllability up to CODimension one ARound $\psi_{1}$}

\subsection{Context for the Nash-Moser theorem}

In this section, we prove Theorem 10 by applying Theorems 7 and 8 with the maps

$$
\Phi\left(\psi_{0}, u\right):=\left(\psi_{0}, \mathcal{P} \psi(T)\right) \quad \text { and } \quad \mathcal{P}\left(\psi_{0}, \widetilde{\psi_{f}}\right):=\left(\mathcal{P} \psi_{0}, \widetilde{\psi_{f}}\right)
$$

for any $\psi_{0} \in L^{2}, \widetilde{\psi_{f}} \in V$ and the spaces

$$
\begin{gathered}
E_{a}:=\left[\mathcal{S} \cap H_{(0)}^{a}((0,1), \mathbb{C})\right] \times \overline{H_{0}^{a / 2}}((0, T), \mathbb{R}), \\
F_{a}:=\left[\mathcal{S} \cap H_{(0)}^{a}((0,1), \mathbb{C})\right] \times\left[B \cap V \cap H_{(0)}^{a}((0,1), \mathbb{C})\right],
\end{gathered}
$$

where $B:=\left\{\varphi \in L^{2}((0,1), \mathbb{C}) ;\|\varphi\|_{L^{2}}<1\right\}$.

6.2. Controllability up to codimension one of the linearized system around $\left(\psi_{1}, u \equiv 0\right)$ with tame estimates

Proposition 13. Let $T:=2 / \pi$. There exists $C>0$ such that, for every $\Psi_{0} \in H_{(0)}^{7}((0,1), \mathbb{C}) \cap T_{\mathcal{S}}\left(\varphi_{1}\right), \widetilde{\Psi_{f}} \in$ $H_{(0)}^{7} \cap V \cap T_{\mathcal{S}}\left(\varphi_{1}\right)$, there exists a trajectory $(\Psi, v)$ of $\left(\Sigma_{0,0}\right)$ with $\Psi(0)=\Psi_{0}, \mathcal{P} \Psi(T)=\widetilde{\Psi_{f}}, v \in \overline{H_{0}^{3}}((0, T), \mathbb{R})$, moreover

$$
\|v\|_{H_{0}^{1}((0, T), \mathbb{R})} \leqslant C\left\|\left(\mathcal{P} \Psi_{0}, \widetilde{\Psi_{f}}\right)\right\|_{H^{3} \times H^{3}} \quad \text { and } \quad\|v\|_{H_{0}^{3}((0, T), \mathbb{R})} \leqslant C\left\|\left(\mathcal{P} \Psi_{0}, \widetilde{\Psi_{f}}\right)\right\|_{H^{7} \times H^{7}}
$$

Proof. Thanks to Section 2, it is sufficient to prove the existence of a constant $C>0$ such that, for every $d=\left(d_{k}\right)_{k \geqslant 2} \in h^{4}$, there exists $v \in \overline{H_{0}^{3}}((0, T), \mathbb{R})$ with the following prescribed Fourier coefficients

$$
\left\{\begin{array}{l}
\int_{0}^{T} \dot{v}(t) \mathrm{d} t=0, \\
\int_{0}^{T} \dot{v}(t) \mathrm{e}^{i\left(\lambda_{k}-\lambda_{1}\right)} \mathrm{d} t=d_{k}, \forall k \geqslant 2
\end{array}\right.
$$

and which satisfies

$$
\|\dot{v}\|_{L^{2}((0, T), \mathbb{R})} \leqslant C\|d\|_{l^{2}} \quad \text { and } \quad\|\dot{v}\|_{H_{0}^{2}((0, T), \mathbb{R})} \leqslant C\|d\|_{h^{4}} .
$$

A suitable candidate is

$$
v(t):=\left(\sum_{k=2}^{\infty} d_{k} \mathrm{e}^{-i\left(\lambda_{k}-\lambda_{1}\right) t}+\text { c.c. }\right)\left(1-\cos \left(\pi^{2} t\right)\right) .
$$

Remark 12. This proposition probably holds with any $T>0$. 


\subsection{Controllability up to codimension one of the linearized system around $(\psi, u)$, close to $\left(\psi_{1}, 0\right)$ in $E_{4}$, with tame estimates}

The aim of this section is the proof of the existence of a right inverse to the differential map $d \Phi\left(\psi_{0}, u\right)$ when $\left(\psi_{0}, u\right)$ is close enough to $\left(\psi_{1}(0), 0\right)$ in $E_{4}$, which satisfies (3.8) and (3.9).

Let $\left(\psi_{0}, u\right) \in E_{8}$, and $\psi$ be the solution of the Cauchy problem

$$
\left\{\begin{array}{l}
i \dot{\psi}=-\psi^{\prime \prime}+\left(\dot{u}-4 u^{2}\right)(t) x^{2} \psi, x \in(0,1), t \in(0, T) \\
\psi(t, 0)=\psi(t, 1)=0 \\
\psi(0)=\psi_{0}
\end{array}\right.
$$

The linearized system around $(\psi, u)$ is

$$
\left(\Sigma_{l}\right)\left\{\begin{array}{l}
i \dot{\Psi}=-\Psi^{\prime \prime}+\left(\dot{u}-4 u^{2}\right)(t) x^{2} \Psi+(\dot{v}-8 u v)(t) x^{2} \psi, x \in(0,1), t \in(0, T) \\
\Psi(t, 0)=\Psi(t, 1)=0 .
\end{array}\right.
$$

Again, we use the distances, for $s=2,4,6,8$,

$$
\delta_{s}:=\left\|\left(\psi_{0}, u\right)-\left(\varphi_{1}, 0\right)\right\|_{E_{s}} .
$$

We want to prove the following proposition.

Proposition 14. Let $T:=2 / \pi$. There exists a constant $C$ such that, when $\delta_{4}$ is small enough, for every $\psi_{0} \in H_{(0)}^{7}((0,1), \mathbb{C}) \cap T_{\mathcal{S}}\left(\varphi_{1}\right)$, for every $\widetilde{\Psi_{f}} \in H_{(0)}^{7}((0,1), \mathbb{C}) \cap V$, there exists a trajectory $(\Psi, v)$ of $\left(\Sigma_{l}\right)$ with $\Psi(0)=\Psi_{0}, \mathcal{P} \Psi(T)=\widetilde{\Psi_{f}}, v \in \overline{H_{0}^{3}}((0, T), \mathbb{C})$ and

$$
\begin{aligned}
&\|v\|_{H_{0}^{1}((0, T), \mathbb{R})} \leqslant C\left[\left\|\left(\mathcal{P} \Psi_{0}, \widetilde{\Psi_{f}}\right)\right\|_{H^{3} \times H^{3}}+\delta_{4}\left\|\left(\Psi_{0}, \widetilde{\Psi_{f}}\right)\right\|_{H^{3} \times H^{3}}\right] \\
&\|v\|_{H_{0}^{3}((0, T), \mathbb{R})} \leqslant C\left[\left\|\left(\mathcal{P} \Psi_{0}, \widetilde{\Psi_{f}}\right)\right\|_{H^{7} \times H^{7}}+\delta_{8}\left\|\left(\Psi_{0}, \widetilde{\Psi_{f}}\right)\right\|_{H^{3} \times H^{3}}\right]
\end{aligned}
$$

Note that it is sufficient to prove the following proposition.

Proposition 15. Let $T:=2 / \pi$. There exists a constant $C$ such that, for every $\widetilde{\Psi_{f}} \in H_{(0)}^{7}((0,1), \mathbb{C}) \cap V$, there exists a trajectory $(\Psi, v)$ of $\left(\Sigma_{l}\right)$ with $\Psi(0)=0, \mathcal{P} \Psi(T)=\widetilde{\Psi_{f}}, v \in \overline{H_{0}^{3}}((0, T), \mathbb{C})$ and

$$
\begin{aligned}
& \|v\|_{H_{0}^{1}((0, T), \mathbb{R})} \leqslant C\left\|\widetilde{\Psi_{f}}\right\|_{H^{3}}, \\
& \|v\|_{H_{0}^{3}((0, T), \mathbb{R})} \leqslant C\left[\left\|\widetilde{\Psi_{f}}\right\|_{H^{7}}+\delta_{8}\left\|\widetilde{\Psi_{f}}\right\|_{H^{3} \times H^{3}}\right] .
\end{aligned}
$$

Proof of Proposition 14 thanks to Proposition 15. We consider the decomposition $\Psi=\Psi_{1}+\Psi_{2}$ where $\mu:=$ $\dot{u}-4 u^{2}$ and

$$
\left\{\begin{array} { l } 
{ i \dot { \Psi } _ { 1 } = - \Psi _ { 1 } ^ { \prime \prime } + \mu ( t ) x ^ { 2 } \Psi _ { 1 } , } \\
{ \Psi ( t , 0 ) = \Psi ( t , 1 ) = 0 , } \\
{ \Psi ( 0 ) = \Psi _ { 0 } , }
\end{array} \quad \left\{\begin{array}{l}
i \dot{\Psi}_{2}=-\Psi_{2}^{\prime \prime}+\mu(t) x^{2} \Psi_{2}+(\dot{v}-8 u v)(t) x^{2} \psi \\
\Psi_{2}(t, 0)=\Psi_{2}(t, 1)=0 \\
\Psi_{2}(0)=0 .
\end{array}\right.\right.
$$


There exist $v \in H_{0}^{3}((0, T), \mathbb{R})$ such that $\mathcal{P} \Psi_{2}(T)=\widetilde{\Psi_{f}}-\mathcal{P} \Psi_{1}(T)$ and

$$
\begin{aligned}
& \|v\|_{H_{0}^{1}((0, T), \mathbb{R})} \leqslant C\left\|\widetilde{\Psi_{f}}-\mathcal{P} \Psi_{1}(T)\right\|_{H^{3}}, \\
& \|v\|_{H_{0}^{3}((0, T), \mathbb{R})} \leqslant C\left[\left\|\widetilde{\Psi_{f}}-\mathcal{P} \Psi_{1}(T)\right\|_{H^{7}}+\delta_{8}\left\|\widetilde{\Psi_{f}}-\mathcal{P} \Psi_{1}(T)\right\|_{H^{3} \times H^{3}}\right] .
\end{aligned}
$$

In order to get the bounds (6.1), let us prove that

$$
\begin{aligned}
& \left\|\mathcal{P} \Psi_{1}(T)\right\|_{H^{3}} \leqslant C\left[\left\|\mathcal{P} \Psi_{0}\right\|_{H^{3}}+\delta_{4}\left\|\Psi_{0}\right\|_{H^{3}}\right], \\
& \left\|\mathcal{P} \Psi_{1}(T)\right\|_{H^{7}} \leqslant C\left[\left\|\mathcal{P} \Psi_{0}\right\|_{H^{7}}+\delta_{8}\left\|\Psi_{0}\right\|_{H^{3}}\right] .
\end{aligned}
$$

We consider the decomposition

$$
\Psi_{1}(t)=\sum_{k=1}^{\infty} x_{k}(t) \varphi_{k, \mu} \quad \text { where } \quad x_{k}(t):=\left\langle\Psi_{1}(t), \varphi_{k, \mu(t)}\right\rangle .
$$

We have

$$
\left.x_{k}(T)=\left\langle\Psi_{0}, \varphi_{k}\right\rangle+\int_{0}^{T} \dot{\mu}(t)\left\langle\Psi_{1}(t), \frac{d \varphi_{k, \gamma}}{\mathrm{d} \gamma}\right]_{\mu(t)}\right\rangle \mathrm{e}^{i \int_{0}^{t} \lambda_{k, \mu(s) \mathrm{d} s} \mathrm{~d} t} .
$$

Thus, using Proposition 9, we get, when $\delta_{4}$ is small enough,

$$
\begin{aligned}
& \left\|\mathcal{P} \Psi_{1}(T)\right\|_{H^{3}} \leqslant C\left(\sum_{k=2}^{\infty}\left|k^{3} x_{k}(T)\right|^{2}\right)^{1 / 2} \leqslant C\left[\left\|\mathcal{P} \Psi_{0}\right\|_{H^{3}}+\delta_{4}\left\|\Psi_{0}\right\|_{H^{3}}\right], \\
& \left\|\mathcal{P} \Psi_{1}(T)\right\|_{H^{7}} \leqslant C\left(\sum_{k=2}^{\infty}\left|k^{7} x_{k}(T)\right|^{2}\right)^{1 / 2} \leqslant C\left[\left\|\mathcal{P} \Psi_{0}\right\|_{H^{7}}+\delta_{4}\left\|\Psi_{0}\right\|_{H^{3}}+\delta_{6}\left\|\Psi_{0}\right\|_{H^{5}}+\delta_{8}\left\|\Psi_{0}\right\|_{H^{3}}\right] .
\end{aligned}
$$

We conclude thanks to the convexity of the norms.

Proof of Proposition 15. Thanks to the decomposition

$$
\Psi(t)=\sum_{k=1}^{\infty} y_{k}(t) \varphi_{k, \mu} \quad \text { where } \quad y_{k}(t):=\left\langle\Psi(t), \varphi_{k, \mu(t)}\right\rangle,
$$

the equality $\mathcal{P} \Psi(T)=\widetilde{\Psi_{f}}$ is equivalent to $M(v)=d$ where $M(v)=\left(M(v)_{k}\right)_{k \geqslant 2}, d=\left(d_{k}\right)_{k \geqslant 2}$ and, for every $k \geqslant 2$,

$$
\begin{gathered}
\left.M(v)_{k}:=\int_{0}^{T}\left(-i(\dot{v}-8 u v)\left\langle x^{2} \psi(t), \varphi_{k, \mu(t)}\right\rangle+\dot{\mu}(t)\left\langle\Psi(t), \frac{\mathrm{d} \varphi_{k, \gamma}}{\mathrm{d} \gamma}\right]_{\mu(t)}\right\rangle\right) \mathrm{e}^{i \int_{0}^{t} \lambda_{k, \mu(s)} \mathrm{d} s} \mathrm{~d} t, \\
d_{k}:=\left\langle\widetilde{\Psi_{f}}, \varphi_{k}\right\rangle .
\end{gathered}
$$

We conclude applying Proposition 6 exactly in the same way as in Section 4.4.

The assumptions of Theorem 8 can be checked in the same way.

\section{Remarks, conjectures}

\subsection{Regularity}

In Theorems 5 and 10 , the assumption $\psi_{0}, \psi_{f} \in H_{(0)}^{5+\epsilon}((0,1), \mathbb{C})$ is only technical, one conjectures that these local controllability results hold in

- $H_{(0)}^{3}((0,1), \mathbb{C})$ with control functions in $L^{2}$;

- $H_{(0)}^{5}((0,1), \mathbb{C})$ with control functions in $H_{0}^{1}$;

- $H_{(0)}^{7}((0,1), \mathbb{C})$ with control functions in $H_{0}^{2}$, etc. 


\subsection{Minimal time for controllability}

For the compactness argument explained in Section 1.5.1, one need local controllability results around $\psi_{\text {ref }}:=$ $\sqrt{1-\theta_{2}-\theta_{3}} \psi_{1}+\sqrt{\theta_{2}} \psi_{2}+\sqrt{\theta_{3}} \psi_{3}$ in time $T$ such that $\psi_{\text {ref }}(0)=\psi_{\text {ref }}(T)$, for instance $T=2 / \pi$ or $4 / \pi$. However, the minimal time for local controllability is another interesting problem.

One conjectures that the local controllability around $\psi_{\text {ref }}$, with $\left(\theta_{2}, \theta_{3}\right) \in \operatorname{Int}(\mathcal{D})$, (i.e. Th. 5) holds in any positive time $T$. Indeed, the linearized system around $\left(\psi_{\text {ref }}, u \equiv 0\right)$ is probably controllable in any time $T>0$ (see Rem. 4). Thus, it is also probably controllable with the bounds (3.8) and (3.9) (corresponding to $v=0$ ) in any time $T>0$. Moreover, the application of the Nash-Moser theorem does not introduce a positive minimal time (see Rem. 9).

One conjectures that the local controllability up to codimension one around $\psi_{1}$ (i.e. Th. 10) holds in any time $T>0$. Indeed, the linearized system around $\left(\psi_{1}, u \equiv 0\right)$ is controllable up to codimension one in any $T>0$ (see Th. 4). Thus, Proposition 13 probably holds with any time $T>0$. Moreover, the application of the Nash-Moser theorem does not introduce a positive minimal time (see Rem. 9).

We think the following assertions are equivalent

- there exists a positive minimal time for the local controllability around $\psi_{1}$;

- there exists of a positive minimal time for the Proposition 11 to hold.

Some computations justify that the condition of Proposition 11 can be written

$$
\sum_{k=2}^{\infty} a_{k}^{2}\left(\lambda_{k}-\lambda_{1}\right)^{3} \Im\left(\int_{0}^{T} v(t) \mathrm{e}^{i\left(\lambda_{1}-\lambda_{k}\right) t} \int_{0}^{t} v(\tau) \mathrm{e}^{i\left(\lambda_{k}-\lambda_{1}\right) \tau} \mathrm{d} \tau \mathrm{d} t\right) \in(0,+\infty)(\text { resp. } \in(-\infty, 0))
$$

It is probably possible to move the second order term instantaneously in one of the two directions $\pm i \psi_{1}(T)$, which means realize $\in(0,+\infty)$ or $\in(-\infty, 0)$ in $(7.1)$ with arbitrarily small $T$. Perhaps the motion in the other direction needs a positive minimal time. A proof by contradiction could also be tried, as in [18].

The existence of a minimal time for moving from $\varphi_{1}$ to $\varphi_{2}$ is also an open problem. The compactness argument used to prove Theorem 1 does not give any clue.

The existence of a minimal time for particular motions on the nonlinear system could be studied directly, for instance by adapting the proof of [17]. In the situation studied in [10], there exists a positive minimal time for the local controllability around $\left(\psi_{1}, s \equiv 0, d \equiv 0\right)$ which has been proved in [17]. This situation is quite different because the linearized system around $\left(\psi_{1}, s \equiv 0, d \equiv 0\right)$ misses an infinite number of directions.

\subsection{Generalizations}

The strategies of this papers can be generalized to Schrödinger equations of the form

$$
i \dot{\psi}=-\psi^{\prime \prime}+u(t) a(x) \psi, x \in(0,1) .
$$

If the function $a$ has some parity property on the space interval $(0,1)$, then the return method can be used, as in $[9,10]$. This method was introduced by Coron in [13] to solve a stabilization problem, it has also been used for controllability problems by Coron in [14-16], by Coron et Fursikov in [19], by Fursikov et Yu. Imanuvilov in [20], by Glass in [21-23], by Horsin in [27] and by Sontag in [36].

Acknowledgements. The author thanks Enrique Zuazua for having attracted her attention to this controllability problem and Jean-Michel Coron for fruitful discussions and advice on this work.

\section{Appendix A: Study of $\varphi_{k, \gamma}$ AND $\lambda_{k, \gamma}$}

In this appendix, we state some useful results on the eigenvalues $\lambda_{k, \gamma}$ and the orthonormal eigenfunctions $\varphi_{k, \gamma}$ of the operator

$$
D\left(A_{\gamma}\right):=H^{2} \cap H_{0}^{1}((0,1), \mathbb{R}), \quad A_{\gamma} \varphi:=\varphi^{\prime \prime}+\gamma x^{2} \varphi .
$$


When $\gamma=0$, we write $\lambda_{k}, \varphi_{k}$ instead of $\lambda_{k, 0}, \varphi_{k, 0}$, and we have

$$
\varphi_{k}(x):=\sqrt{2} \sin (n \pi x), \quad \lambda_{n}:=(n \pi)^{2} .
$$

The functions $\gamma \mapsto \varphi_{k, \gamma}$ and $\gamma \mapsto \lambda_{k, \gamma}$ are analytic (see [29], Motzkin-Taussky theorem p. 85),

$$
\varphi_{k, \gamma}=\varphi_{k}+\gamma \varphi_{k}^{(1)}+\gamma^{2} \varphi_{k}^{(2)}+\gamma^{3} \varphi_{k}^{(3)}+\ldots, \quad \lambda_{k, \gamma}=\lambda_{k}+\gamma \lambda_{k}^{(1)}+\gamma^{2} \lambda_{k}^{(2)}+\gamma^{3} \lambda_{k}^{(3)}+\ldots
$$

Proposition 16. For every $k \in \mathbb{N}^{*}$, we have

$$
\begin{aligned}
& \left\{\begin{array}{l}
-\frac{\mathrm{d}^{2}}{\mathrm{~d} x^{2}} \varphi_{k}^{(1)}+x^{2} \varphi_{k}=\lambda_{k} \varphi_{k}^{(1)}+\lambda_{k}^{(1)} \varphi_{k}, \\
\varphi_{k}^{(1)}(0)=\varphi_{k}^{(1)}(1)=0, \\
\int_{0}^{1} \varphi_{k}(q) \varphi_{k}^{(1)}(q) \mathrm{d} q=0 .
\end{array}\right. \\
& \lambda_{k}^{(1)}=\left\langle x^{2} \varphi_{k}, \varphi_{k}\right\rangle=\frac{1}{3}-\frac{1}{2(k \pi)^{2}}, \quad \varphi_{k}^{(1)}=\sum_{j=1, j \neq k}^{\infty} x_{k, j} \varphi_{j}, \quad x_{k, j}:=\frac{(-1)^{k+j} 8 k j}{\pi^{4}(k+j)^{3}(k-j)^{3}}, \forall j \neq k .
\end{aligned}
$$

There exists a constant $C>0$ such that, for every $k \in \mathbb{N}^{*}$

$$
\left\|\varphi_{k}^{(1)}\right\|_{L^{2}} \leqslant \frac{C}{k}
$$

Proof. Using (A.1), we get

$$
\left\|\varphi_{k}^{(1)}\right\|_{L^{2}}=\frac{8 k}{\pi^{4}}\left(\sum_{j=1, j \neq k}^{\infty} \frac{j^{2}}{(k+j)^{6}(k-j)^{6}}\right)^{1 / 2} \leqslant \frac{C}{k} .
$$

Corollary 2. There exists $\gamma^{*}>0, C^{*}>0$ such that, for every $\gamma_{1} \in\left(-\gamma^{*}, \gamma^{*}\right)$, for every $k \in \mathbb{N}^{*}$, we have

$$
\begin{gathered}
\left\|\varphi_{k, \gamma_{1}}-\varphi_{k}\right\|_{H^{s}((0,1), \mathbb{R})} \leqslant C^{*}\left|\gamma_{1}\right| k^{s-1}, \text { for every integers } \in[0,4], \\
\left|\lambda_{k, \gamma_{1}}-\lambda_{k}\right| \leqslant C\left|\gamma_{1}\right| . \\
\left|\frac{1}{\lambda_{k, \gamma_{1}}}-\frac{1}{\lambda_{k}}\right| \leqslant \frac{C\left|\gamma_{1}\right|}{k^{4}} .
\end{gathered}
$$

Proposition 17. For every $k \in \mathbb{N}^{*}$, we have

$$
\begin{gathered}
\left\{\begin{array}{l}
-\frac{\mathrm{d}^{2}}{\mathrm{~d} x^{2}} \varphi_{k}^{(2)}+x^{2} \varphi_{k}^{(1)}=\lambda_{k} \varphi_{k}^{(2)}+\lambda_{k}^{(1)} \varphi_{k}^{(1)}+\lambda_{k}^{(2)} \varphi_{k}, \\
\varphi_{k}^{(2)}(0)=\varphi_{k}^{(2)}(1)=0, \\
\int_{0}^{1}\left(2 \varphi_{k}(q) \varphi_{k}^{(2)}+\varphi_{k}^{(1)} \varphi_{k}^{(1)}\right)(q) \mathrm{d} q=0,
\end{array}\right. \\
\lambda_{k}^{(2)}=\left\langle x^{2} \varphi_{k}^{(1)}, \varphi_{k}\right\rangle, \quad \varphi_{k}^{(2)}=\sum_{j=1}^{\infty} y_{k, j} \varphi_{j}, \quad y_{k, k}:=-\frac{1}{2}\left\|\varphi_{k}^{(1)}\right\|_{L^{2}}^{2}, \quad y_{k, j}:=\frac{\left\langle x^{2} \varphi_{k}^{(1)}, \varphi_{j}\right\rangle-\lambda_{k}^{(1)} x_{k, j}}{\lambda_{k}-\lambda_{j}}, \forall j \neq k .
\end{gathered}
$$

There exists a constant $C>0$ such that, for every $k \in \mathbb{N}^{*}$

$$
\begin{gathered}
\left\|\varphi_{k}^{(2)}\right\|_{L^{2}} \leqslant \frac{C}{k}, \\
\left|\lambda_{k}^{(2)}\right| \leqslant \frac{C}{k^{2}} .
\end{gathered}
$$


Proof. Using (A.5), we get

$$
\left\|\varphi_{k}^{(2)}\right\|_{L^{2}} \leqslant \frac{1}{2}\left\|\varphi_{k}^{(1)}\right\|_{L^{2}}^{2}+\frac{C}{k}\left(\sum_{j=1, j \neq k}^{\infty} \frac{1}{(k-j)^{2}(k+j)^{2}}\right)^{1 / 2}+C k\left(\sum_{j=1, j \neq k}^{\infty} \frac{j^{2}}{(k+j)^{8}(k-j)^{8}}\right)^{1 / 2} \leqslant \frac{C}{k^{2}} .
$$

The explicit expression of $\varphi_{k}^{(1)}$ gives

$$
\left|\lambda_{k}^{(2)}\right|=C\left|\sum_{j \neq k} \frac{k^{2} j^{2}}{(k+j)^{5}(k-j)^{5}}\right| \leqslant \frac{C}{k^{2}} .
$$

The quantities $\varphi_{k, \gamma}, \lambda_{k, \gamma}$ are analytic functions of the parameter $\gamma$ in a neighborhood of zero, thus, we can consider their derivatives with respect to $\gamma$. We denote

$$
\left.\frac{\mathrm{d}^{j} \varphi_{k, \gamma}}{\mathrm{d} \gamma^{j}}\right]_{\gamma_{1}}
$$

the $j^{\text {th }}$ derivative of the map $\gamma \mapsto \varphi_{k, \gamma}$ evaluated at the point $\gamma=\gamma_{1}$ and

$$
\lambda_{k, \gamma_{1}^{\prime}}, \lambda_{k, \gamma}^{\prime \prime}
$$

the second and third derivatives of the function $\gamma \mapsto \lambda_{k, \gamma}$ evaluated at the point $\gamma=\gamma_{1}$.

Corollary 3. For every $\gamma_{1} \in \mathbb{R}$, we have

$$
\left.\left.A_{\gamma_{1}} \frac{\mathrm{d} \varphi_{k, \gamma}}{\mathrm{d} \gamma}\right]_{\gamma_{1}}+x^{2} \varphi_{k, \gamma_{1}}=\lambda_{k, \gamma_{1}} \frac{\mathrm{d} \varphi_{k, \gamma}}{\mathrm{d} \gamma}\right]_{\gamma_{1}}+\lambda_{k, \gamma_{1}}^{\prime} \varphi_{k, \gamma_{1}} .
$$

There exists $\gamma^{*}$ and $C^{*}>0$ such that, for every $\gamma_{1} \in\left(-\gamma^{*}, \gamma^{*}\right)$, for every $k \in \mathbb{N}^{*}$, we have

$$
\begin{gathered}
\left.\left.\| \frac{\mathrm{d} \varphi_{k, \gamma}}{\mathrm{d} \gamma}\right]_{\gamma_{1}}-\frac{\mathrm{d} \varphi_{k, \gamma}}{\mathrm{d} \gamma}\right]_{0} \|_{H^{s}((0,1), \mathbb{R}} \leqslant C^{*}\left|\gamma_{1}\right| k^{s-2} \text { for every integer } s \in[0,4] \\
\left.\| \frac{\mathrm{d} \varphi_{k, \gamma}}{\mathrm{d} \gamma}\right]_{\gamma_{1}} \|_{H^{s}((0,1), \mathbb{R})} \leqslant C^{*} k^{s-1} \\
\left\|\varphi_{k, \gamma_{1}}-\varphi_{k}-\gamma_{1} \varphi_{k}^{(1)}\right\|_{L^{2}((0,1), \mathbb{R})} \leqslant \frac{C^{*}\left|\gamma_{1}\right|^{2}}{k^{2}} \\
\left|\lambda_{k, \gamma_{1}}^{\prime}-\lambda_{k}^{\prime}\right| \leqslant \frac{C\left|\gamma_{1}\right|}{k} \\
\left|\lambda_{k, \gamma_{1}}-\lambda_{k}-\frac{\gamma}{3}\right| \leqslant \frac{C|\gamma|}{k^{2}}
\end{gathered}
$$

Proof. Thanks to (A.6) and (A.1), we have

$$
\left|\lambda_{k, \gamma_{1}}-\lambda_{k}-\gamma_{1}\left(\frac{1}{3}-\frac{1}{2(k \pi)^{2}}\right)\right| \leqslant \frac{C\left|\gamma_{1}\right|^{2}}{k^{2}},
$$

which gives (A.12). 
Proposition 18. There exists $C^{*}>0$ such that, for every $k \in \mathbb{N}^{*}$,

$$
\left\|\varphi_{k}^{(3)}\right\|_{L^{2}} \leqslant \frac{C^{*}}{k^{2}}
$$

Thus, there exists $\gamma^{*}>0$ such that, for every $k \in \mathbb{N}^{*}$, we have

$$
\left.\| \frac{\mathrm{d}^{2} \varphi_{k, \gamma}}{\mathrm{d} \gamma^{2}}\right]_{\gamma_{1}} \|_{L^{2}} \leqslant \frac{C^{*}}{k^{2}}
$$

\section{ApPEndix B: REgularity and BOUnds on the SOLUtions of THE NONLINEAR System}

This section is dedicated to the statement of existence and regularity results together with bounds on the solution of the Cauchy problem

$$
\left\{\begin{array}{l}
i \dot{\psi}(t, x)=-\psi^{\prime \prime}(t, x)+\left[\dot{u}-4 u^{2}\right](t) x^{2} \psi(t, x)+f(t, x), \\
\psi(t, 0)=\psi(t, 1)=0 \\
\psi(0, x)=\psi_{0}(x)
\end{array}\right.
$$

We use the spaces $E_{a}, a=2,4,6$ defined in Section 3, and the group of isometries of $L^{2}((0,1), \mathbb{C})$ defined by

$$
\mathcal{T}(t) \varphi:=\sum_{k=1}^{\infty}\left\langle\varphi, \varphi_{k}\right\rangle \mathrm{e}^{-i \lambda_{k} t} \varphi_{k}
$$

Proposition 19. Let $T>0, \psi_{0} \in H_{(0)}^{2}((0,1), \mathbb{C}), \mu \in L^{1}((0, T), \mathbb{R})$ and $f \in L^{1}\left((0, T), H_{(0)}^{2}((0,1), \mathbb{R})\right)$. There exists a unique $\psi \in C^{0}\left([0, T], H_{(0)}^{2}((0,1), \mathbb{C})\right)$ which satisfies the following equality in $H_{(0)}^{2}((0,1), \mathbb{C})$ for every $t \in[0, T]$,

$$
\psi(t):=\mathcal{T}(t) \psi_{0}+\int_{0}^{t} \mathcal{T}(t-s)\left[-i \mu(s) x^{2} \psi(s)-i f(s)\right] \mathrm{d} s .
$$

Moreover, there exists a constant $C>0$ such that

$$
\|\psi\|_{C^{0}\left([0, T], H_{(0)}^{2}\right)} \leqslant\left[\left\|\psi_{0}\right\|_{H_{(0)}^{2}}+\|f\|_{L^{1}\left((0, T), H_{(0)}^{2}\right)}\right] \mathrm{e}^{C\|\mu\|_{L^{1}}} .
$$

Proof. The existence come from a fixed point argument when $\|\mu\|_{L^{1}((0, T), \mathbb{R})}$ is small enough. Otherwise, we use a partition $[0, T]=\cup I_{j}, 1 \leqslant j \leqslant N$ such that $\|p\|_{L^{1}\left(I_{j}, \mathbb{R}\right.}$ is small enough so that the previous result holds. The bound relies on Gronwall's Lemma.

Applying recursively this result, we get the following propositions

Proposition 20. Let $T>0, \psi_{0} \in H_{(0)}^{4}((0,1), \mathbb{C}), u \in W^{2,1}((0, T), \mathbb{R})$ and $f \in W^{1,1}\left((0, T), H_{(0)}^{2}((0,1), \mathbb{R})\right)$. The solution $\psi$ of (B.2), with $\mu:=\dot{u}-4 u^{2}$, belongs to $C^{1}\left([0, T], H_{(0)}^{2}\right) \cap C^{0}\left([0, T], H_{(0)}^{4}\right)$, it is a solution of (B.1) in the sense of Definition 1. Moreover, for every $r>0$, there exists a constant $C(r)>0$ such that, for every $\left(\psi_{0}, u\right) \in E_{4}$ with $\left\|\left(\psi_{0}, u\right)\right\|_{E_{4}} \leqslant r$ and for every $f \in W^{1,1}\left((0, T), H_{(0)}^{2}\right)$, the quantities $\|\psi\|_{C^{0}\left([0, T], H_{(0)}^{4}\right)}$ and $\|\dot{\psi}\|_{C^{0}\left([0, T], H_{(0)}^{2}\right)}$ are bounded by

$$
C(r)\left[\left\|\psi_{0}\right\|_{H_{(0)}^{4}}+\|f\|_{W^{1,1}\left((0, T), H_{(0)}^{2}\right)}\right] .
$$

Proposition 21. Let $T>0, \psi_{0} \in H_{(0)}^{6}((0,1), \mathbb{C}), u \in W^{3,1}((0, T), \mathbb{R})$ be such that $\left(\dot{u}-4 u^{2}\right)(0)=0$ and

$$
\left.f \in W^{2,1}\left((0, T), H_{(0)}^{2}((0,1), \mathbb{R})\right) \cap C^{0}\left([0, T], H^{4}((0,1), \mathbb{R})\right)\right) .
$$


The solution $\psi$ of (B.2), with $\mu:=\dot{u}-4 u^{2}$ belongs to $C^{2}\left([0, T], H_{(0)}^{2}\right) \cap C^{1}\left([0, T], H^{4}\right) \cap C^{0}\left([0, T], H^{6}\right)$ If $\mu(T)=0$ and $f(T) \in H_{(0)}^{4}((0,1), \mathbb{C})$ then $\psi(T) \in H_{(0)}^{6}((0,1), \mathbb{C})$. Moreover, for every $r>0$, there exists a constant $C(r)>0$ such that, for every $\left(\psi_{0}, u\right) \in E_{6}$ with $\left\|\left(\psi_{0}, u\right)\right\|_{E_{4}} \leqslant r$ and for every $f$ with (B.3), the quantities $\|\psi\|_{C^{0}\left([0, T], H^{6}\right)},\|\dot{\psi}\|_{C^{0}\left([0, T], H^{4}\right)}$ and $\|\ddot{\psi}\|_{C^{0}\left([0, T], H^{2}\right)}$ are bounded by

$$
C(r)\left[\left\|\psi_{0}\right\|_{H^{6}}+\|u\|_{H^{3}}\left\|\psi_{0}\right\|_{H_{(0)}^{2}}+\|f\|_{W^{2,1}\left((0, T), H_{(0)}^{2}\right)}+\|f\|_{C^{0}\left([0, T], H^{4}\right)}+\|u\|_{H^{3}}\|f\|_{L^{1}\left((0, T), H_{(0)}^{2}\right)}\right] .
$$

\section{REFERENCES}

[1] F. Albertini and D. D'Alessandro, Notions of controllability for bilinear multilevel quantum systems. IEEE Trans. Automat. Control 48 (2003) 1399-1403.

[2] S. Alinhac and P. Gérard, Opérateurs pseudo-différentiels et théorème de Nash-Moser. Intereditions (Paris), collection Savoirs actuels (1991).

[3] C. Altafini, Controllability of quantum mechanical systems by root space decomposition of su(n). J. Math. Phys. 43 (2002) 2051-2062.

[4] J.M. Ball, J.E. Marsden and M. Slemrod, Controllability for distributed bilinear systems. SIAM J. Control Optim. 20 (1982).

[5] L. Baudouin, A bilinear optimal control problem applied to a time dependent Hartree-Fock equation coupled with classical nuclear dynamics. Portugaliae Matematica (N.S.) 63 (2006) 293-325.

[6] L. Baudouin and J. Salomon, Constructive solution of a bilinear control problem. C.R. Math. Acad. Sci. Paris 342 (2006) $119-124$.

[7] L. Baudouin, O. Kavian and J.-P. Puel, Regularity for a Schrödinger equation with singular potential and application to bilinear optimal control. J. Differential Equations 216 (2005) 188-222.

[8] K. Beauchard, Local controllability of a 1-D beam equation. SIAM J. Control Optim. (to appear).

[9] K. Beauchard, Local Controllability of a 1-D Schrödinger equation. J. Math. Pures Appl. 84 (2005) 851-956.

[10] K. Beauchard and J.-M. Coron, Controllability of a quantum particle in a moving potential well. J. Functional Analysis 232 (2006) 328-389.

[11] R. Brockett, Lie theory and control systems defined on spheres. SIAM J. Appl. Math. 25 (1973) 213-225.

[12] E. Cancès, C. Le Bris and M. Pilot, Contrôle optimal bilinéaire d'une équation de Schrödinger. C.R. Acad. Sci. Paris, Série I 330 (2000) 567-571.

[13] J.-M. Coron, Global asymptotic stabilization for controllable systems without drift. Math. Control Signals Systems 5 (1992) $295-312$.

[14] J.-M. Coron, Contrôlabilité exacte frontière de l'équation d'Euler des fluides parfaits incompressibles bidimensionnels. $C . R$. Acad. Sci. Paris 317 (1993) 271-276.

[15] J.-M. Coron, On the controllability of 2-D incompressible perfect fluids. J. Math. Pures Appl. 75 (1996) 155-188.

[16] J.-M. Coron, Local Controllability of a 1-D Tank Containing a Fluid Modeled by the shallow water equations. ESAIM: COCV 8 (2002) 513-554.

[17] J.-M. Coron, On the small-time local controllability of a quantum particule in a moving one-dimensional infinite square potential well. C.R. Acad. Sci., Série I 342 (2006) 103-108.

[18] J.-M. Coron and E. Crépeau, Exact boundary controllability of a nonlinear KdV equation with critical lengths. J. Eur. Math. Soc. 6 (2004) 367-398.

[19] J.-M. Coron and A. Fursikov, Global exact controllability of the 2D Navier-Stokes equation on a manifold without boundary. Russ. J. Math. Phys. 4 (1996) 429-448.

[20] A.V. Fursikov and O.Yu. Imanuvilov, Exact controllability of the Navier-Stokes and Boussinesq equations. Russian Math. Surveys 54 (1999) 565-618.

[21] O. Glass, On the controllability of the 1D isentropic Euler equation. J. European Mathematical Society 9 (2007) $427-486$.

[22] O. Glass, Exact boundary controllability of 3-D Euler equation. ESAIM: COCV 5 (2000) 1-44.

[23] O. Glass, On the controllability of the Vlasov-Poisson system. J. Differential Equations 195 (2003) 332-379.

[24] G. Gromov, Partial Differential Relations. Springer-Verlag, Berlin-New York-London (1986).

[25] A. Haraux, Séries lacunaires et contrôle semi-interne des vibrations d'une plaque rectangulaire. J. Math. Pures Appl. 68 (1989) $457-465$.

[26] L. Hörmander, On the Nash-Moser Implicit Function Theorem. Annales Academiae Scientiarum Fennicae (1985) $255-259$.

[27] T. Horsin, On the controllability of the Burgers equation. ESAIM: COCV 3 (1998) 83-95.

[28] R. Ilner, H. Lange and H. Teismann, Limitations on the control of Schrödinger equations. ESAIM: COCV 12 (2006) 615-635.

[29] T. Kato, Perturbation Theory for Linear operators. Springer-Verlag, Berlin, New-York (1966).

[30] W. Krabs, On moment theory and controllability of one-dimensional vibrating systems and heating processes. Springer - Verlag (1992).

[31] I. Lasiecka and R. Triggiani, Optimal regularity, exact controllability and uniform stabilization of Schrödinger equations with Dirichlet controls. Differential Integral Equations 5 (1992) 571-535. 
[32] I. Lasiecka, R. Triggiani and X. Zhang, Global uniqueness, observability and stabilization of nonconservative Schrödinger equations via pointwise Carlemann estimates. J. Inverse Ill Posed-Probl. 12 (2004) 183-231.

[33] G. Lebeau, Contrôle de l'équation de Schrödinger. J. Math. Pures Appl. 71 (1992) 267-291.

[34] Machtyngier, Exact controllability for the Schrödinger equation. SIAM J. Contr. Opt. 32 (1994) 24-34.

[35] M. Mirrahimi and P. Rouchon, Controllability of quantum harmonic oscillators. IEEE Trans. Automat. Control 49 (2004) $745-747$.

[36] E. Sontag, Control of systems without drift via generic loops. IEEE Trans. Automat. Control 40 (1995) 1210-1219.

[37] G. Turinici, On the controllability of bilinear quantum systems, in Mathematical Models and Methods for Ab Initio Quantum Chemistry, C. Le Bris and M. Defranceschi Eds., Lect. Notes Chemistry 74, Springer (2000).

[38] E. Zuazua, Remarks on the controllability of the Schrödinger equation. CRM Proc. Lect. Notes 33 (2003) $193-211$. 